Preprints of the

Max Planck Institute for

Research on Collective Goods

Bonn 2017/10

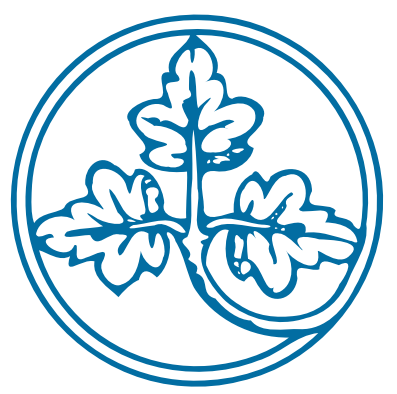

Optimal income taxation with labor supply responses at two margins: When is an Earned Income Tax Credit optimal?

Emanuel Hansen

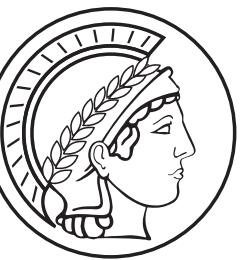




\section{Optimal income taxation with labor supply responses at two margins: When is an Earned Income Tax Credit optimal?}

Emanuel Hansen

May 2017 


\title{
Optimal income taxation with labor supply responses at two margins: When is an Earned Income Tax Credit optimal?
}

\author{
Emanuel Hansen*
}

May 18, 2017

\begin{abstract}
This paper studies optimal non-linear income taxation in an empirically plausible model with labor supply responses at the intensive (hours, effort) and the extensive (participation) margin. In this model, redistributive taxation gives rise to a previously neglected trade-off between two aspects of efficiency: To reduce the deadweight loss from distortions at the extensive margin, the social planner has to increase distortions at the intensive margin and vice versa. Due to this trade-off, minimizing the overall deadweight loss requires to distort labor supply by low-skill workers upwards at both margins. Building on these insights, the paper is the first to provide conditions under which social welfare is maximized by an Earned Income Tax Credit with negative marginal taxes and negative participation taxes at low income levels.
\end{abstract}

Keywords: Optimal income taxation, Extensive margin, Intensive margin JEL classification: H21; H23; D82

${ }^{*}$ University of Cologne, Center for Macroeconomic Research, Albertus-Magnus-Platz, D-50923 Köln, Germany. Email: hansen@wiso.uni-koeln.de. The author thanks Felix Bierbrauer, Martin Hellwig, Sebastian Koehne, Wojciech Kopczuk, Etienne Lehmann, Thorsten Louis, Dominik Sachs, Paul Schempp, Joel Slemrod, Dezsö Szalay and John Weymark as well as participants at the TTC 2016 in Toulouse, the IIPF Doctoral School 2015 in Warsaw, the IIPF 2014 in Lugano, the VfS 2014 in Hamburg and at seminars in Bonn and Cologne for helpful comments and discussions. The usual disclaimer applies. 


\section{Introduction}

Governments in all developed countries use labor income taxes and income-based transfers to redistribute resources from the rich to the poor. The properties of these tax-transfer schemes differ substantially across countries, though, especially with respect to the treatment of low incomes. Most European countries provide more or less generous payments to unemployed agents and smaller, decreasing payments to low-income earners. Economists refer to these tax-transfer schemes with strictly positive marginal taxes and strictly positive participation taxes 11 as Negative Income Taxes (NIT). Other countries use tax-transfer schemes that provide higher transfers to low-income earners than to unemployed agents. The most prominent example is given by the Earned Income Tax Credit (EITC) in the US, which involves both negative marginal taxes and negative participation taxes for low-income earners..$^{2}$ On the one hand, there seems to be a growing consensus among political practitioners that the EITC is an effective instrument for fighting poverty and should be further expanded $:^{3}$ On the other hand, economists have so far struggled to identify conditions under which such an income tax schedule is optimal in the standard sense of maximizing social welfare 4 The present paper fills this gap by providing sufficient and necessary conditions for the optimality of an EITC with both negative marginal taxes and negative participation taxes.

The common approach to determine the optimal income tax involves, first, the definition of a welfare function that provides a rationale for redistribution from the rich to the poor, and second, the maximization of this welfare function over the set of (non-linear) income taxes that satisfy the government's budget constraint, taking into account the agents' labor supply responses. In most previous models, the agents respond to tax changes either at the intensive margin only, i.e., by adjusting their hours worked or their effort spent while working, or at the extensive margin only, i.e., by entering or leaving the formal labor market. Both classes of models are inconsistent with the empirical evidence that labor supply responds at the intensive margin as well as the extensive margin: "the world is obviously a mix of the two models" (Saez 2002: p. 1054). More specifically, empirical studies consistently find

\footnotetext{
${ }^{1}$ The participation tax function assigns to each income level $y>0$ the difference between tax levels at income $y$ and at zero income, $T(y)-T(0)$.

2 Nichols \& Rothstein (2015) provide a detailed description of the details of the EITC and a comprehensive review of the recent literature studying its labor supply effects.

${ }^{3}$ In particular, both President Obama and Paul Ryan-then Republican Chairman of the House of Representatives Budget Committee-proposed to roughly double the maximum EITC payments for childless workers (see Executive Office 2014, House Budget Committee 2014).

${ }^{4}$ Most previous papers find that the optimal income tax is unambiguously given by an NIT. I comment below on the most important exceptions, including Saez (2002).
} 
that extensive-margin responses are particularly important at the bottom of the income distribution: The participation elasticity of low-income earners is both larger than their elasticity of hours worked, and larger than the participation elasticity of medium-income earners and high-income earners (see, e.g., Juhn et al. 1991, 2002, Meghir \& Phillips 2010) $:^{5}$ The present paper investigates the optimal redistributive tax-transfer scheme in a model that is consistent with these empirical patterns.

In particular, I study a two-dimensional screening model in which the agents face both marginal costs of providing output as in Mirrlees (1971) and fixed costs of working as in Diamond (1980). The agents are privately informed about their fixed costs of working and their skills, where the latter determine the marginal costs of output provision. To make the model tractable, I follow the random participation approach by Rochet \& Stole (2002) and assume additive separability between the fixed-cost component and the other components of the utility function. The analysis focuses on the empirically and economically relevant cases in which, first, society has a concern for redistribution from higher-skilled to lower-skilled agents and, second, participation elasticities are decreasing over the skill dimension.

The paper uses this model to contribute in three ways to the literature on optimal income taxation. First, it provides sufficient conditions for the optimality of an EITC, expressed in terms of the model's primitives: utility functions, type distributions, and the properties of the social welfare function. In particular, it shows that the optimal income tax is given by an EITC with negative marginal taxes and negative participation taxes at low income levels if (a) society has strong concerns for redistribution from the rich to the poor, but only limited concerns for local redistribution from the poor to the very poor and (b) there is a sufficiently large mass of highly productive agents..$^{6}$

The previous literature has neither identified conditions under which an EITC with negative marginal taxes as well as negative participation taxes is optimal, nor clarified whether it can be optimal at all under reasonable assumptions. Surprisingly, only a few papers have studied optimal income taxation in a model with labor supply responses at both margins so far. Most related to the present paper are Saez (2002) and Jacquet et al. (2013). Saez (2002) is the first to strongly advocate and investigate this setting. He shows that an EITC with negative marginal taxes

\footnotetext{
${ }^{5}$ For additional empirical evidence on how participation elasticities vary across the population, see Meyer \& Rosenbaum (2001), Eissa \& Hoynes (2004), Immervoll et al. (2007), Blau \& Kahn (2007) and the surveys by Hotz \& Scholz (2003), Eissa \& Hoynes (2006), McClelland \& Mok (2012).

${ }^{\circ}$ This result holds whenever labor supply responds at both margins, and higher-income earners respond more elastically at the extensive margin than lower-income earners. These patterns are ensured by a set of reasonably weak assumptions on the agents' utility functions and the type distributions. Besides, the paper also provides sufficient conditions for the optimality of an NIT.
} 
for low-income workers can be optimal if both the participation elasticities and the optimal participation taxes for all higher-income earners are sufficiently large. He concludes that "optimal tax rates are not necessarily nonnegative as in the pure intensive model" (Saez 2002, p. 1055), but does not clarify in which cases these conditions are indeed met-especially, in which cases the endogenous levels of optimal participation taxes are large enough. The general perception of Saez results seems to be that an EITC can only be optimal if labor supply responds more strongly at the extensive margin than at the intensive margin (see, e.g., Brewer et al. 2010, Piketty \& Saez 2013).7 In contrast, Jacquet et al. (2013) demonstrate that the signs of optimal tax rates depend on how the strength of participation responses varies over the skill distribution. Their main results are given by sufficient conditions for the optimality of positive marginal taxes and positive participation taxes as in the NIT, expressed in terms of participation (semi-)elasticities and social welfare weights. They do not provide results on when the optimal marginal taxes are negative as in the EITC.

Second, the paper proposes a new strategy to analytically solve multi-dimensional screening models. The major problem in solving these models is that the set of binding incentive-compatibility (IC) constraints is a priori unclear. Jacquet et al. (2013) show that this problem can sometimes be circumvented. In particular, they identify conditions under which all downwards IC constraints along the skill dimension are binding and the optimal marginal taxes are positive everywhere, just as in Mirrlees (1971). In contrast, an EITC with negative marginal taxes can only be optimal in cases for which at least some upwards IC constraints are binding. Hence, I cannot use the method proposed by Jacquet et al. (2013) to identify sufficient conditions for the optimality of an EITC, but have to develop a new solution strategy.

The methodological innovation of this paper is to study a hybrid model with a continuous set of fixed cost types and a discrete set of skill types in the first step, and to focus on skill sets with sufficiently small distances between adjacent skill sets in the second step. In particular, the discrete skill set has two advantages: First, the optimal tax problem involves a finite number of distinguishable downwards and upwards IC constraints, which can be added or deleted one by one to study partially relaxed problems. Second, there exist allocations in which both local IC constraints are slack for some pairs of adjacent skill types 8 The proofs of my main results exploit both properties, and would hence not be valid with a continuous skill set as in Jacquet et al. (2013).

\footnotetext{
${ }^{7}$ The analysis in the present paper does not confirm this perception.

${ }^{8}$ With a continuous skill set, downwards and upwards IC constraints are collapsed into an envelope condition that always has to be satisfied with equality.
} 
The discrete skill set has a major drawback, however. If there is a strictly positive distance between adjacent skill types, it is impossible to check whether a potentially optimal allocation satisfies a particular IC constraint unless strong functional form assumptions are imposed. I solve this problem by verifying incentive compatibility when the difference between adjacent skill types converges to zero or, more precisely, marginally increases from zero. This allows me to determine unambiguously which IC constraints are binding whenever the skill set is sufficiently "dense", i.e., the distance between adjacent skill types is strictly positive but small. Intuitively, I study the behavior of the model at the transition between a discrete skill set and a continuous skill set, exploiting major advantages of both model classes.

Third, the paper provides a novel intuition for the potential optimality of an EITC. In particular, it shows that the optimality of negative marginal taxes is driven by an inherent trade-off between labor supply distortions at both margins, which has not been elucidated in the previous literature. The following thought experiment helps to understand this trade-off and its implications. Consider an economy populated by agents who differ both in their skills (very low, low, or high) and in their fixed costs of working, so that some agents in each skill group choose to remain unemployed for each tax schedule. Assume that the social planner wants to redistribute some fixed, strictly positive amount of resources from the rich (highskilled workers) to the poor (unemployed agents, very-low-skill workers and low-skill workers) in such a way that efficiency is maximized, i.e., the deadweight loss from labor supply distortions at both margins is minimized 9 Hence, he does not care for how the resources are distributed among the poor. The properties of the efficiencymaximizing redistribution scheme can be explained in two steps.

For the first step, assume that the social planner only seeks to minimize the labor supply distortions at the extensive margin (given some amount of redistribution). If he increases the transfer to the unemployed, some workers in all three skill groups find it attractive to leave the labor market and save the fixed costs of working. If he increases the transfers to both groups of lower-skill workers, some unemployed agents in these skill groups find it attractive to enter the labor market, but none of the high-skill agents has an incentive to leave the labor market. Hence, the second option induces less distortions at the extensive margin. Accordingly, the efficiency-maximizing tax schedule involves higher transfers to both groups of lowerskill workers than to the unemployed, i.e., negative participation taxes ${ }^{10}$

But how should these transfers be divided between both groups of lower-skill

\footnotetext{
${ }^{9} \mathrm{~A}$ formal definition of the deadweight loss (or excess burden) is provided in Section 3

${ }^{10}$ These arguments are closely related to those in papers on optimal income taxation with labor supply responses at the extensive margin only (see, e.g., Saez 2002 and Christiansen 2015).
} 
workers? To minimize the distortions at the extensive margin, the planner has to apply a version of the classical inverse elasticity rule. If the very-low-skill agents respond more elastically at the extensive margin than all higher-skilled agents (in line with the empirical evidence), the planner should pay smaller transfers to the very-low-skill workers than to the low-skill workers. For this purpose, he has to introduce negative marginal taxes in the relevant income range.

For the second step, assume that the social planner also seeks to minimize the labor supply distortions at the intensive margin. The lower-skilled workers respond to the negative marginal taxes mentioned above by increasing their output provision, so that labor supply becomes upwards distorted at the intensive margin. Hence, the efficiency-maximizing planner faces a trade-off between labor supply distortions at both margins: To reduce the deadweight loss from upward distortions at the extensive margin, he has to increase the upward distortions at the intensive margin and vice versa. ${ }^{11}$ To implement the optimal compromise between both types of distortions, the planner has to introduce an EITC with negative participation taxes and negative marginal taxes.

A more rigorous derivation of this result is provided in Section 6. The section also explains when, and why, an EITC continues to be optimal if, first, the planner's redistributive concerns among the poor and, second, the incentive-compatibility constraints between high-skill workers and low-skill workers are taken into account.

The paper proceeds as follows. In Section 2, I briefly review the previous literature on optimal income taxation. In Section 3, I introduce the model and the optimal tax problem. Section 4 introduces three important assumptions on the primitives of the model, while Section 5 presents the results of this paper. In Section 6, I explain the economic mechanism underlying these results, focusing on the trade-off between labor supply distortions at both margins. I conclude in Section 7. All formal proofs are provided in Appendix $\mathrm{A}{ }^{12}$

\section{Related literature}

The previous literature on optimal non-linear income taxation studies mainly two classes of models that differ in the type of costs agents face and, correspondingly, the margin at which they respond to tax changes.

\footnotetext{
${ }^{11}$ To avoid the distortions at the intensive margin, the planner would have to set marginal taxes equal to zero, i.e., identical transfers to both groups of low-skill workers. As explained above, however, this would lead to a larger deadweight loss from distortions at the extensive margin.

${ }^{12}$ Appendices B and C provide supplementary results, graphical illustrations and an example with commonly used functional forms.
} 
First, a large number of studies follow the seminal paper by Mirrlees (1971) by assuming that the agents face only variable costs of providing effort, which are affected by a single private parameter referred to as skill. In these models, labor supply responds to tax changes at the intensive (hours, effort) margin only. The "central result" (Hellwig 2007: 1449) in this strand of the literature is that the optimal income tax is an NIT with strictly positive marginal taxes almost everywhere ${ }^{13}$ This result holds whenever the welfare function gives rise to a desire for redistributing resources from higher-skilled to lower-skilled agents (see, amongst others, Seade 1977, 1982, Hellwig 2007). Complementing these findings, Choné \& Laroque (2010) and Brett \& Weymark (2017) show that negative marginal taxes as in the EITC can be optimal in the intensive model if the social planner has a non-standard desire to redistribute resources from lower-income earners to higher-income earners ${ }^{14}$ Notably, the same results apply whether the skill set is continuous or discrete (see

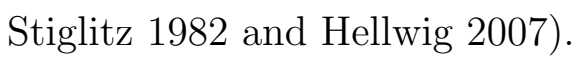

Second, a smaller set of papers follow Diamond (1980) by studying models in which the agents differ not only in their skills, but also in their privately observable fixed costs of working. This strand of the literature was revived by Saez (2002) and a series of papers by Laroque (2005) and Choné \& Laroque (2005, 2011). In their models, labor supply responds to tax changes at the extensive (participation) margin only. As there are no variable costs of providing output, all agents either prefer to work at full capacity or to be unemployed. The papers typically find that optimal participation taxes at the bottom of the income distribution are negative if and only if the social planner cares almost as much for the low-skilled workers as for the unemployed (see Diamond 1980, Saez 2002, Laroque 2005, Choné \& Laroque 2005, 2011, Christiansen 2015). While optimal marginal taxes can also be computed, they are economically irrelevant as they do not lead to distortions at the intensive margin. Again, the results do not depend on whether the skill set is continuous as in Choné \& Laroque (2011) or discrete as in Christiansen (2015).

Finally, there exist a few papers that study optimal income taxation with labor supply responses at both margins. Saez (2002) strongly advocates the mixed model due to its empirical relevance and discusses how the mechanisms of this model differ from the pure intensive and the pure extensive model. Most importantly, he is

\footnotetext{
${ }^{13}$ The optimal marginal income tax is zero at the top and, under certain conditions, at the bottom of the income distribution.

${ }^{14}$ In Choné \& Laroque (2010), the desire to redistribute resources from the poor to the rich arises if higher-skilled agents are on average disadvantaged in another private parameter that also affects the marginal costs of output provision. Brett \& Weymark (2017) investigate income taxes that maximize the utility of workers with an intermediate skill type, i.e., that redistribute as many resources as possible to medium-skill workers from below as well as from above.
} 
the first to show that negative marginal taxes at low-income levels are compatible with a standard desire for redistribution if both the participation elasticities and the optimal participation taxes for higher-income earners are sufficiently large. This crucial insight does not allow to verify the optimality of an EITC, however, because the optimal participation taxes are themselves endogenous entities that depend on the redistributive concerns, the labor supply elasticities and the optimal marginal taxes 15

The paper by Jacquet et al. (2013) is most closely related to my paper. They also study a random participation model in which the agents differ in fixed costs of working and in skills, where the latter parameter affects the marginal costs of providing output. The main difference to my model is that they consider a type set that is continuous in both dimension. In contrast, I study a hybrid model with a discrete set of skill types and a continuous set of fixed cost types ${ }^{16}$ The main result of Jacquet et al. (2013) is given by a sufficient condition for the optimality of positive marginal taxes. While this condition is expressed in terms of endogenous entities-participation elasticities and marginal social weights-as acknowledged by the authors, they also provide examples for it is unambiguously satisfied ${ }^{17}$ For example, they show that the optimal income tax is given by an NIT if (a) the social planner maximizes a Rawlsian welfare function and (b) higher-skilled workers respond more elastically at the extensive margin that lower-skilled workers, in line with the empirical evidence 18

Lorenz \& Sachs (2012) study a model that is similar in spirit to the one by Jacquet et al. (2013), although the labor supply responses at the extensive margin result from a minimum hours constraint instead of fixed costs of working. They complement the literature by showing that optimal participation taxes as in the NIT are positive whenever the tax designer cares less for the lowest-skilled workers than for the average agent in the population.

In contrast to my paper, the previous literature has not identified conditions under which an EITC with negative marginal taxes as well as negative participation taxes is optimal, nor has it clarified whether such a scheme can be optimal at

\footnotetext{
${ }^{15}$ Additionally, Saez numerically solves the optimal income tax for commonly used functional forms, finding positive marginal taxes and (sometimes) negative participation taxes at the bottom.

${ }^{16}$ Besides, their analysis is somewhat more general in allowing for income effects in labor supply.

${ }^{17}$ In particular, Jacquet et al. (2013) show that the optimal marginal tax is positive at all income levels if the function $h(\omega):=[1-\alpha(\omega)] / \eta(\omega)$ is strictly increasing in $\omega$, where $\alpha(\omega)$ is the marginal social weight and $\eta(\omega)$ is the semi-elasticity of participation for agents with skill type $\omega$.

${ }^{18}$ Lehmann et al. (2014) use a two-country random-participation model to study non-linear income taxation with labor supply responses at the intensive (hours) margin and the migration margin. Although their model differs from the ones in Jacquet et al. (2013) and the present paper in many aspects and leads to different conclusions, the mechanisms at work are similar.
} 
all under empirically plausible assumptions. Moreover, it has not provided a clear economic intuition for why negative marginal taxes could be expected to be optimal given a standard concern for redistribution from the rich to the poor. In particular, none of the previous papers comments on the trade-off between labor supply distortions at both margins, which I document in section $6.1{ }^{19}$

Finally, Beaudry et al. (2009) rationalize in-work benefit schemes as the EITC in a two-dimensional screening model that deviates in several aspects from the previously discussed literature. First, the agents do not face fixed costs of working, but opportunity costs related to the possibility of generating income in an informal (black) labor market. Hence, the social planner holds a desire to redistribute resources from unemployed agents (the workers in the informal sector) to formally employed agents with identical skills. Second, the planner is able to observe hours worked in the formal sector and, consequently, to condition tax payments on the wages of formally employed agents. Due to these two properties and in contrast to my model, an EITC with negative marginal taxes is always optimal for agents earning wages below some cutoff wage, and the optimal transfers to unemployed agents are always zero. 20

\section{Model}

The following subsection presents a two-dimensional screening model in which labor supply responds to tax changes at the intensive and at the extensive margin. Subsection 3.2 provides a formal definition of the optimal tax problem and of labor supply distortions at both margins. Subsection 3.3 explains how the optimal allocation can be decentralized via non-linear income taxes, and Subsection 3.4 discusses the relation between social welfare functions and marginal social welfare weights.

\subsection{The economy}

The set of agents is given by a continuum of mass one and denoted by $I$, with typical element $i$. Agent $i$ 's consumption is denoted by $c^{i}$, his contribution to the economy's output by $y^{i}$. Agent $i$ derives utility from consumption and suffers from the cost of providing output. This cost can be separated into a variable effort cost and a fixed

\footnotetext{
${ }^{19}$ In the model by Saez (2002), it is unclear what a distortion in labor supply is and how it can be measured (because the costs of output provision are not modeled explicitly). Jacquet et al. (2013) do not distinguish explicitly between labor supply distortions at both margins.

${ }^{20} \mathrm{In}$ Beaudry et al. (2009), agents can respond at the intensive as well as the extensive margin. However, the optimal allocation only involves labor supply distortions at the extensive margin, as all agents work in only one sector (except for the agents with a single threshold skill type).
} 
cost of participating in the labor market. Formally, individual preferences can be represented by the following utility function: ${ }^{21}$

$$
u\left(c^{i}, y^{i} ; \omega^{i}, \delta^{i}\right)=c^{i}-h\left(y^{i}, \omega^{i}\right)-\mathbb{1}_{y^{i}>0} \delta^{i} .
$$

The fixed cost of participating in the labor market is given by an individual parameter $\delta^{i} \in \Delta$, which I refer to as agent $i$ 's fixed cost type. The variable effort cost of providing output is measured by the function $h$. It depends on the output level $y^{i}$ and an individual parameter $\omega^{i} \in \Omega$, which I refer to as $i$ 's skill type. Absolute and marginals cost of providing output are both decreasing in this parameter, so that $h_{\omega}\left(y^{i}, \omega^{i}\right)<0$ and $h_{y \omega}\left(y^{i}, \omega^{i}\right)<0$ for all $y^{i}>0$ and $\omega^{i} \in \Omega$. Moreover, $h$ is strictly increasing and strictly convex in $y^{i}$, so that $h_{y}\left(y^{i}, \omega^{i}\right)>0$ and $h_{y y}\left(y^{i}, \omega^{i}\right)>0$ for all $y^{i}>0$ and $\omega^{i} \in \Omega$. Finally, $h$ is assumed to satisfy $h_{y y \omega}(y, \omega) \leq 0$ for all $y^{i}>0$ and $\omega^{i} \in \Omega$, and $h\left(0, \omega^{i}\right)=0$ as well as the Inada conditions $\lim _{y \rightarrow 0} h_{y}\left(y^{i}, \omega^{i}\right)=0$ and $\lim _{y \rightarrow \infty} h_{y}\left(y^{i}, \omega^{i}\right)=\infty$ for all $\omega^{i} \in \Omega$.

Agent $i$ is privately informed about his skill type $\omega^{i}$ and his fixed cost type $\delta^{i}$. The skill set $\Omega$ is given by a finite ordered set $\left\{\omega_{1}, \omega_{2}, \ldots, \omega_{n}\right\}$ with $\omega_{j+1} / \omega_{j} \geq 1+\varepsilon$ for all $j \in\{1,2, \ldots, n-1\}$ and some $\varepsilon>0$. The set of fixed costs $\Delta$ is given by a closed interval with lower endpoint $\underline{\delta}$ and upper endpoint $\bar{\delta}$. These endpoints are assumed to satisfy

$$
\begin{aligned}
& \underline{\delta}<\max _{y>0} y-h\left(y, \omega_{1}\right) \text { and } \\
& \bar{\delta}>\max _{y>0} y-h\left(y, \omega_{n}\right) .
\end{aligned}
$$

Under laissez-faire, agents with fixed cost type $\underline{\delta}$ and any skill type $\omega \in \Omega$ would thus provide positive output, while agents with fixed cost type $\bar{\delta}$ and any skill type $\omega \in \Omega$ would provide zero output. ${ }^{22}$ As will become clear below, the combination of a discrete set of skills and a continuous set of fixed costs helps to explain the interaction between labor supply distortions at both margins.

The joint cross-section distribution of the pair $\left(\omega^{i}, \delta^{i}\right)$ in the population at large is commonly known and denoted by $K: \Omega \times \Delta \rightarrow[0,1]$. The share of agents with skill type $\omega_{j}$, which I henceforth refer to as skill group $j$, is given by the number $f_{j}>0$ for any $j \in j$. The distribution function of fixed cost types in any skill group $j \in J$ is twice continuously differentiable and denoted by $G_{j}$. The corresponding density function $g_{j}$ is bounded from below by some number $\underline{g}>0$ for all $\delta \in \Delta$ and there exists some closed subset of $\Delta$ on which $g_{j}$ is weakly decreasing.

\footnotetext{
${ }^{21}$ I comment on the implications of the functional form imposed by (1) below.

${ }^{22}$ This ensures that tax changes may affect the participation decisions of agents in all skill groups.
} 


\subsection{The optimal tax problem}

I use a mechanism design approach to solve for the optimal non-linear income tax. Thus, I study the problem to maximize a social welfare function (to be defined below) over the set of feasible and incentive-compatible allocations. An allocation is given by two functions $c: \Omega \times \Delta \rightarrow \mathbb{R}$ and $y: \Omega \times \Delta \rightarrow \mathbb{R}_{0}^{+}$that specify the consumption and output levels for all types in $\Omega \times \Delta$. It is feasible if overall consumption does not exceed overall output, i.e.,

$$
\int_{\Omega \times \Delta} c(\omega, \delta) d K(\omega, \delta) \leq \int_{\Omega \times \Delta} y(\omega, \delta) d K(\omega, \delta),
$$

and incentive-compatible if

$$
u(c(\omega, \delta), y(\omega, \delta) ; \omega, \delta) \geq u\left(c\left(\omega^{\prime}, \delta^{\prime}\right), y\left(\omega^{\prime}, \delta^{\prime}\right) ; \omega, \delta\right)
$$

for all types $(\omega, \delta)$ and $\left(\omega^{\prime}, \delta^{\prime}\right)$ in $\Omega \times \Delta$.

Below, I will investigate whether the optimal allocation involves labor supply distortions. The characterization of these distortions is based on the following thought experiment, which I illustrate in Figures 2 and 3 in Appendix B. Consider an initial allocation in which agent $i$ 's bundle is given by $\left(c^{i}, y^{i}\right) \geq 0$. Now consider providing agent $i$ with a different bundle $(\tilde{c}, \tilde{y}) \geq 0$ such that $\tilde{y}-y^{i}=\tilde{c}-c^{i} \neq 0$. The set of these potential deviations is given by a straight line through $\left(c^{i}, y^{i}\right)$ with slope equal to 1 , the economy's marginal rate of transformation between consumption and output. Agent $i$ 's labor supply is said to be distorted if there is a bundle $(\tilde{c}, \tilde{y})$ on this line that $i$ strictly prefers to $\left(c^{i}, y^{i}\right)$.

First, it might be possible to increase $i$ 's utility through a marginal deviation from $\left(c^{i}, y^{i}\right)$. This will be the case if and only if $i$ 's marginal rate of substitution, $h_{y}\left(y^{i}, \omega^{i}\right)$, differs from 1 . If $h_{y}\left(y^{i}, \omega^{i}\right)<1, i$ would strictly prefer an outputincreasing deviation. Then, $i$ 's labor supply is said to be downwards distorted at the intensive margin. Correspondingly, if $h_{y}\left(y^{i}, \omega^{i}\right)>1, i$ would strictly prefer an output-decreasing deviation, and $i$ 's labor supply is said to be upwards distorted at the intensive margin.

Second, it might be possible to increase $i$ 's utility through a large deviation from $\left(c^{i}, y^{i}\right)$ that changes his participation status (from zero output to positive output or vice versa). Consider an initial allocation with $y^{i}=0$ and the deviation to bundle $\left(c^{i}+\tilde{y}, \tilde{y}\right)$ for some $\tilde{y}>0$. Agent $i$ would be strictly better off with the new bundle than with his initial bundle if and only if $i$ 's total costs of providing output $\tilde{y}$ are below the additional utility from consuming $\tilde{y}, h\left(\tilde{y}, \omega^{i}\right)+\delta^{i}<\tilde{y}$. Hence, $i$ 's labor 
supply is said to be downwards distorted at the extensive margin if both $y^{i}=0$ and $\delta^{i}<\delta^{*}\left(\omega^{i}\right):=\max _{y>0} y-h\left(y, \omega^{i}\right)$.

Correspondingly, consider an initial allocation with $y^{i}>0$. Agent $i$ would be strictly better off with bundle $\left(c^{i}-y^{i}, 0\right)$ than with his initial bundle if and only if $i$ 's total costs of providing output $y^{i}$ exceed the utility from consuming $y^{i}, h\left(y^{i}, \omega^{i}\right)+$ $\delta^{i}>y^{i}$. Hence, $i$ 's labor supply is said to be upwards distorted at the extensive margin if both $y^{i}>0$ and $\delta^{i}>y^{i}-h\left(y^{i}, \omega^{i}\right)$.

These definitions allow to characterize the distortions in the labor supply of each type in $\Omega \times \Delta$ separately. In the interest of readability, however, I will use a more concise characterization of labor supply distortions across the type set. This is possible because only specific patterns of distortions are compatible with implementability and (second-best) Pareto efficiency.

Lemma 1. Every allocation $(c, y)$ that is implementable and Pareto-efficient (in the set of implementable allocations) is characterized by two vectors $\left(y_{j}\right)_{j=1}^{n},\left(c_{j}\right)_{j=0}^{n}$ such that,

- for each $j \in J=\{1,2, \ldots, n\}$, all agents with skill type $\omega_{j}$ and fixed cost type $\delta \leq \delta_{j}:=c_{j}-h\left(y_{j}, \omega_{j}\right)-c_{0}$ receive bundle $\left(c_{j}, y_{j}\right)$, and

- all other agents receive bundle $\left(c_{0}, 0\right)$.

By Lemma 1, any implementable allocation involves pooling by $n+1$ sets of different types. First, all agents with skill type $\omega_{j} \in \Omega$ and fixed cost types below the (endogenous) participation threshold $\delta_{j}$ provide the same output level $y_{j}>0$ and receive the same consumption level $c_{j}>0$. Second, all other agents provide zero output and receive the same consumption level $c_{0}$.

Both properties are driven by the additive separability of the fixed cost component $\delta$ in utility function (1), which follows the random participation approach Rochet \& Stole (2002). First, conditional on not working, an agent's payoff is independent of his type. Hence, incentive compatibility requires that all unemployed agents must receive the same bundle $\left(c_{0}, 0\right)$. Second, conditional on working, an agent's preference relation over any set of bundles depends only on his skill type $\omega$. Hence, all working agents with skill type $\omega_{j} \in \Omega$ must receive the same gross payoff $c-h(y, \omega)$ (but not necessarily the same consumption-output bundle). Consequently, there is a unique fixed cost level $\delta_{j}$ that makes an agent with skill type $\omega_{j}$ indifferent between working and unemployment. Finally, Pareto efficiency ensures that all working agents with skill type $\omega_{j}$ receive the same bundle $\left(c_{j}, y_{j}\right)$ instead of different bundles related to the same gross payoff. 
As indicated before, Lemma 1 allows to concisely characterize the labor supply distortions in any implementable allocation for an entire skill group $j \in J$. The statement that labor supply in skill group $j$ is distorted at the intensive margin characterizes an allocation in which the marginal rate of substitution $h\left(y_{j}, \omega_{j}\right)$ differs from one for all working agents with skill type $\omega_{j}$. The statement that labor supply in skill group $j$ is distorted at the extensive margin characterizes an allocation in which the skill-specific participation threshold $\delta_{j}$ is either located below $\delta^{*}\left(\omega_{j}\right)$ (downward distortion) or above $y_{j}-h\left(y_{j}, \omega_{j}\right)$ (upward distortion) ${ }^{23}$

An allocation is said to be efficient if it does not involve labor supply distortions at any margin, based on the vectors $\left(y_{j}\right)_{j=1}^{n}$ and $\left(\delta_{j}\right)_{j=1}^{n}$. The efficiency losses in all other allocations can be evaluated based on the implied deadweight loss, defined as

$$
\begin{aligned}
D W L(c, y):= & \sum_{j=1}^{n} f_{j} \int_{\underline{\delta}}^{\delta^{*}\left(\omega_{j}\right)} g_{j}(\delta)\left[\delta^{*}\left(\omega_{j}\right)-\delta\right] d \delta \\
& -\sum_{j=1}^{n} f_{j} \int_{\underline{\delta}}^{\delta_{j}} g_{j}(\delta)\left[y_{j}-h\left(y_{j}, \omega_{j}\right)-\delta\right] d \delta,
\end{aligned}
$$

where $\delta_{j}=c_{j}-h\left(y_{j}, \omega_{j}\right)-c_{0}$ as derived in Lemma $1{ }^{24}$

\subsection{Decentralization}

The previous section explains the problem of maximizing welfare over the set of implementable allocations via direct mechanisms. How does this serve the goal of this paper, i.e., investigating the properties of the optimal income tax schedule? First, the taxation principle ensures that every implementable allocation $(c, y)$ can be decentralized through an admissible tax function $T$. Second, as the following section will demonstrate, the labor supply distortions in any allocation $(c, y)$ have straightforward implications for the properties of the tax schedule that decentralizes $(c, y)$-in particular, for the signs of the marginal tax $T^{\prime}(y)$ and the participation tax $T^{P}(y)=T(y)-T(0)$ at all relevant levels of $y$.

Consider the class of social choice functions that are decentralized through nonlinear income tax schedules, mapping output levels into tax payments. I denote by $\left(c_{T}, y_{T}\right)$ the social choice function that is decentralized by tax function $T$. The literature also refers to $y_{T}$ as pre-tax income, and to $c_{T}$ as post-tax income. Tax function

\footnotetext{
${ }^{23}$ Note that their is a conceptual difference between both statements: the second statement refers to an allocation in which labor supply by some but not all agents with skill type $\omega_{j}$ is distorted at the extensive margin. In the interest of readability, I nevertheless use the same terminology to characterize labor supply distortions at both margins.

${ }^{24}$ In Appendix B, I explain the derivation of the deadweight loss formula and show that the overall deadweight loss can be decomposed in efficiency losses from distortions at both margins.
} 
$T$ is admissible if the tax revenue is non-negative, $\int_{\Omega \times \Delta} T\left[y_{T}(\omega, \delta)\right] d K(\omega, \delta) \geq 0$.

The problem of an agent with type $(\omega, \delta)$ is to choose income $y$ in order to maximize $U(c, y ; \omega, \delta)$, subject to the individual budget constraint $c=y-T(y)$. To simplify the exposition, assume that $T: \mathbb{R}_{0}^{+} \rightarrow \mathbb{R}$ is continuously differentiable. Then, the solution to this program is given by

$$
y_{T}(\omega, \delta)=\left\{\begin{array}{cl}
y_{T}^{*}(\omega) & \text { if } \delta \leq \delta_{T}(\omega) \\
0 & \text { if } \delta>\delta_{T}(\omega)
\end{array}\right.
$$

where $y_{T}^{*}(\omega)$ is implicitly defined by

$$
1-T^{\prime}\left[y_{T}^{*}(\omega)\right]=h_{y}\left(y_{T}^{*}(\omega), \omega\right),
$$

and $\delta_{T}(\omega):=y_{T}^{*}(\omega)-h\left(y_{T}^{*}(\omega), \omega\right)-\left[T\left(y_{T}^{*}(\omega)\right)-T(0)\right]$.

The reason for the case distinction in (7) is the following. Conditional on labor market participation, $y>0$, the agent's maximization problem is globally concave. Hence, the conditional optimum $y_{T}^{*}(\omega)$ is characterized by the first-order condition (8), which involves only his skill type $\omega$ and the marginal tax $T^{\prime} 25$ For an agent with type $(\omega, \delta)$, participating in the labor market can increase his utility at most by $\delta_{T}(\omega)$, the net gain from increased consumption and increased effort costs. If his fixed $\operatorname{cost} \delta$ is lower than $\delta_{T}(\omega)$, the agent's individually optimal income level $y_{T}(\omega, \delta)$ coincides with the conditional optimum $y_{T}^{*}(\omega)$. If instead his fixed cost $\delta$ exceeds $\delta_{T}(\omega)$, the agent prefers zero income to any positive income $y>0$. Note that both the fixed cost type $\delta$ and the participation tax $T(y)-T(0)$ affect only the extensive decision whether or not to work at all, but not the intensive decision how much to work conditional on participation.

Now, fix an implementable and Pareto-efficient allocation $(c, y)$. By Lemma 1. any such allocation consists of $n+1$ bundles of consumption and output for the groups of working agents with any skill type $\omega_{j} \in \Omega$ and the group of unemployed agents. Which properties does the tax schedule $T$ need to decentralize this allocation so that $y_{T}(\omega, \delta)=y(\omega, \delta)$ and $c_{T}(\omega, \delta)=c(\omega, \delta)$ for all types in $\Omega \times \Delta$ ?

First, assume that labor supply in skill group $j$ is downwards distorted at the intensive margin, i.e., the marginal rate of substitution at $y_{j}=y_{T}^{*}\left(\omega_{j}\right)$ is below 1 . As shown above, $y_{T}^{*}\left(\omega_{j}\right)$ satisfies $h_{y}\left(y_{T}^{*}\left(\omega_{j}\right), \omega_{j}\right)=1-T^{\prime}\left(y_{T}^{*}\left(\omega_{j}\right)\right)$. Hence, the marginal tax at the income level $y_{T}^{*}\left(\omega_{j}\right)=y_{j}$ must be strictly positive. Intuitively, a positive marginal tax is required to ensure that each agent with skill type $\omega_{j}$ provides lower

\footnotetext{
${ }^{25}$ If $T$ is not continuously differentiable, $y_{T}^{*}(\omega)$ might be located at a kink of $T$ and fail to satisfy the first-order condition (8).
} 
output than efficient. Correspondingly, if and only if labor supply in skill group $j$ is upwards distorted at the intensive margin, the marginal tax at the income level $y_{T}^{*}\left(\omega_{j}\right)=y_{j}$ must be strictly negative.

Second, assume that labor supply in skill group $j$ is downwards distorted at the extensive margin, i.e., $\delta_{j}<\delta^{*}\left(\omega_{j}\right)$. As shown above, an agent self-selects zero income if his fixed cost type $\delta$ exceeds the utility gain of participation, $y-h(y, \omega)-$ $[T(y)-T(0)]$, for all positive income levels. Hence, the participation tax $T(y)-T(0)$ must be strictly positive at the income level that maximizes $y-h\left(y, \omega_{j}\right)$. Intuitively, a positive participation tax is required to ensure that the agent prefers to stay inefficiently out of the labor market. Correspondingly, if labor supply in skill group $j$ is upwards distorted at the extensive margin, the participation tax at income level $y_{T}^{*}(\omega)=y_{j}$ must be strictly negative.

The main results of this paper in Section 5 characterize the optimal labor supply distortions at the intensive margin and the extensive margin. As shown here, these results can straightforwardly be translated into statements about the signs of the optimal marginal tax and the optimal participation tax.

\subsection{The social welfare function}

In optimal tax theory, social welfare is usually taken to be an increasing function of individual utilities that gives rise to a "desire for redistribution" (Hellwig 2007). A standard assumption is that the social objective can be expressed as

$$
\int_{\Omega \times \Delta} \gamma(\omega, \delta) \Psi\left(c(\omega, \delta)-h[y(\omega, \delta), \omega]-\mathbb{1}_{y(\omega, \delta)>0} \delta\right) d K(\omega, \delta),
$$

where the transformation $\Psi: \mathbb{R} \rightarrow \mathbb{R}$ is strictly increasing and weakly concave, and the weighting function $\gamma: \Omega \times \Delta \rightarrow \mathbb{R}_{+}$is weakly decreasing in $\omega$ and weakly increasing in $\delta$. The desire for redistribution is either introduced through transformation $\Psi$ or through type-dependent weights $\gamma$; with quasi-linear preferences, it would not be present if welfare were given by the unweighted sum of individual utilities.

Assume in the following that society associates the same weight $\gamma(\omega, \delta)$ to each type $(\omega, \delta) \in \Omega \times \Delta$. Then, the concavity of $\Psi$ implies that it would be beneficial to redistribute resources from types enjoying high levels of utility to types with lower levels of utility, if one could ignore incentive considerations.

In an economy with heterogeneity in skills only, an agent's utility as well as his output are monotonically increasing in his skill type. Hence, the concavity of $\Psi$ pins down society's preferences for redistributing resources from higher-income earners to lower-income earners, given equal weights $\gamma(\omega, \delta)$ for all types in $\Omega \times \Delta$. 
For the economy with two-dimensional heterogeneity considered here, an agent's utility is increasing in his skill type and decreasing in his fixed cost type. Consequently, redistribution from high-skilled to low-skilled agents is no longer equivalent to redistribution from agents with high utility to agents with low utility. Hence, the concavity of $\Psi$ is neither a necessary nor a sufficient condition for a social desire to redistribute resources from higher-income earners to lower-income earners. ${ }^{26}$ Instead, the direction of desired redistribution depends on the properties of $\Psi$ (and $\gamma$ ) as well as on the joint type distribution $K$.

The basic reason behind this ambiguity is that, by Lemma 1, any implementable allocation involves pooling of $n$ subsets of types that enjoy different utility levels. In particular, working agents with the same skill type $\omega_{j}$, but different fixed cost types must always receive the same bundle. Hence, there is no possibility to redistribute resources between the agents in this set, although this would be socially desirable. In contrast, redistribution is only possible between the $n+1$ groups of agents who provide different output levels: the group of unemployed workers and the groups of workers with skill type $\omega_{j}$ for any $j \in J$. As usual, incentive-compatibility and the single-crossing condition ensure that higher-skilled workers provide larger output levels than lower-skilled workers, $y_{j+1}>y_{j}$, for all $j \in J \backslash\{n\}$. In contrast to the model with one-dimensional heterogeneity, however, the composition of these groups is endogenous to the tax schedule $T$. In particular, $T$ affects the threshold $\delta_{j}$ for each $j \in J$.

Following many other tax papers, I can express the social concerns for redistributing resources in my model by the average social welfare weights associated to the $n+1$ groups mentioned above, evaluated at the welfare-maximizing allocation. For any $j \in J$, I define the social weight $\bar{\alpha}_{j}$ as the marginal welfare effect of increasing $c_{j}$, the consumption level enjoyed by all workers with skill type $\omega_{j}$,

$$
\bar{\alpha}_{j}:=E_{\delta}\left[\gamma\left(\omega_{j}, \delta\right) \Psi^{\prime}\left(c_{j}-h\left(y_{j}, \omega_{j}\right)-\delta\right) \mid \delta \leq \delta_{j}\right]
$$

Correspondingly, I define the social weight $\bar{\alpha}_{0}$ of unemployed agents as the marginal effect from increasing $c_{0}$, the consumption level of all unemployed agents,

$$
\bar{\alpha}_{0}:=E_{\omega_{j}, \delta}\left[\gamma\left(\omega_{j}, \delta\right) \Psi^{\prime}\left(c_{0}\right) \mid \delta<\delta_{j}, j \in J\right] .
$$

\footnotetext{
${ }^{26}$ Similarly, if $\gamma$ is decreasing in $\omega$ and increasing in $\delta$, this does not ensure a social desire for redistribution from higher-income earners to lower-income earners.
} 
The average weight across the population is given by

$$
\bar{\alpha}_{M}:=\int_{\Omega \times \Delta}\left[\left(\bar{\alpha}_{j}-\bar{\alpha}_{0}\right) \mathbb{1}_{y(\omega, \delta)>0}+\bar{\alpha}_{0}\right] d K\left(\omega_{j}, \delta\right)
$$

In the remainder of this paper, I restrict my attention to welfare functions that give rise to monotonically decreasing social weight sequences, which seems to be most natural and economically most relevant case.27 Importantly, this restriction simplifies the comparison of my results with those in the standard Mirrlees framework: If the optimal tax has non-standard properties, they cannot be driven by non-monotonicities in the social weights, but must be related to the interaction of labor supply responses at the intensive and extensive margins.

To simplify the exposition, I henceforth treat the group-specific social weights as if they were exogenous objects and denote them by $\alpha=\left(\alpha_{0}, \alpha_{1}, \ldots, \alpha_{n}\right)$. In particular, Propositions 2 to 4 provide conditions on $\alpha$ for which the optimal income tax is either given by an EITC or by an NIT. Propositions 5 (in the main text), 8 and 9 (in Appendix B.6) clarify, however, that these conditions are indeed satisfied by the welfare weights that endogenously arise for some well-behaved welfare functions. Without loss of generality, I will focus on the set of strictly decreasing social weight sequences that are normalized to have an average weight of 1 . I denote this set of weight sequences by $\mathcal{A}$.

\section{Assumptions}

In the following, I will impose three sets of conditions on the economy. These include some standard regularity conditions that are commonly used in the literature on optimal income taxation. Other conditions have not been used in this literature so far. The specific role of each condition is discussed in the subsequent sections.

The first condition imposes restrictions on the joint type distributions, expressed in terms of the hazard rates of fixed costs distributions. Fix a skill type $\omega_{j}$. Recall that $G_{j}$ denotes the $c d f$ of the distribution of fixed cost types in the group of agents with this skill type. The hazard rate of this $c d f$ is given by $A_{j}(\delta):=\frac{g_{j}(\delta)}{G_{j}(\delta)}$.

Condition 1. The joint type distribution has the following properties:

(i) For every $j \in J, A_{j}(\delta)$ is strictly decreasing in $\delta$.

(ii) For every $j \in J_{-n}:=J \backslash\{n\}$ and $\delta \in \Delta, A_{j}(\delta) \geq A_{j+1}(\delta)$.

\footnotetext{
${ }^{27}$ In Appendix B.3. I provide conditions on $\Psi, \gamma$ and $K$ that jointly ensure that $\bar{\alpha}$ is monotonically decreasing. Additionally, I provide an example in which, despite a strictly concave $\Psi$, the social weight sequence is locally increasing.
} 
Condition 1 requires the hazard rates to be strictly decreasing along the fixed cost dimension, and weakly decreasing along the skill dimension. Part (i) is a standard monotone hazard rate condition. It is satisfied whenever the skill-dependent distribution $G_{j}$ of fixed costs is log-concave, which is true for most commonly used distribution functions. Part (ii) rules out a specific type of positive joint variation between skill types and fixed cost types. For some distribution functions, it is identical to the assumption that $G_{j}$ weakly dominates $G_{j+1}$ in the sense of first-order stochastic dominance 28

Jointly, both parts of Condition 1 ensure that low-skill workers respond more elastically at the extensive margin than high-skill workers, in line with the empirical findings by Juhn et al. (1991, 2002) and Meghir \& Phillips (2010). As will become clear, the main results of this paper depend crucially on this pattern. It proves helpful to measure these extensive-margin responses by the semi-elasticity $\eta_{j}$ of participation in each skill group $j$, formally defined by

$$
\eta_{j}(c, y):=\frac{\partial G_{j}\left(c_{j}-h\left(y_{j}, \omega_{j}\right)-c_{0}\right)}{\partial c_{j}} \frac{1}{G_{j}\left(c_{j}-h\left(y_{j}, \omega_{j}\right)-c_{0}\right)}=\frac{g_{j}\left(\delta_{j}\right)}{G_{j}\left(\delta_{j}\right)}
$$

for each $j \in J{ }^{29}$ Under Condition 1, the relative sizes of these semi-elasticities across different skill types are consistent with the empirical evidence.

Lemma 2. For each $j \in J_{-n}$, skill group $j$ has a strictly larger semi-elasticity of participation than skill group $j+1, \eta_{j}(c, y)>\eta_{j+1}(c, y)$, in every implementable allocation.

The second condition imposes further restrictions on the skill-dependent distributions of fixed costs. In particular, it compares the previously defined $c d f$ hazard rate $A_{j}(\delta)$ with the hazard rate of the corresponding $p d f$ in the same skill group $j$. I denote this $p d f$ hazard rate by $a_{j}:=\frac{g_{j}^{\prime}(\delta)}{g_{j}(\delta)}$.

Condition 2. The joint type distribution has the following properties:

(i) For every $j \in J, a_{j}(\delta)$ is weakly decreasing in $\delta$ with $\frac{d a_{j}(\delta)}{d \delta} \in\left[2 \frac{d A_{j}(\delta)}{d \delta}, 0\right]$.

(ii) For every $j \in J_{-n}$ and $\delta \in \Delta, 0 \leq a_{j}(\delta)-a_{j+1}(\delta) \leq 2\left[A_{j}(\delta)-A_{j+1}(\delta)\right]$.

\footnotetext{
${ }^{28}$ In general, however, part (iii) is neither stronger nor weaker than the assumption of first-order stochastic dominance.

${ }^{29}$ More precisely, $\eta_{j}$ represents the semi-elasticity of the skill-specific participation share $G_{j}\left(\delta_{j}\right)$ with respect to the net-of-tax income $c_{j}=y_{j}-T\left(y_{j}\right)$ faced by the workers with skill type $\omega_{j}$. It indicates by how much percent the participation share in skill group $j$ increases if $c_{j}$ is increased by one unit. In contrast, the (standard) elasticity of participation measures by how much percent the participation share in skill group $j$ increases if $c_{j}$ is increased by one percent.
} 
Condition 2 imposes two novel conditions that have not been used in the literature before. They require that the $p d f$ hazard rate $a_{j}$ varies across both type dimensions in the same direction as the $c d f$ hazard rate $A_{j}$, but at a sufficiently small rate compared to the latter. By part (ii), the $p d f$ hazard rate must be monotonically decreasing in $\delta$ at a sufficiently small pace. By part (ii), the difference between the $p d f$ hazard rates in two adjacent skill groups must be small enough compared to the difference in the $c d f$ hazard rates. Note that both parts of Condition 2 are satisfied, e.g., if the fixed costs are uniformly distributed in all skill groups. Moreover, the second condition trivially holds whenever skill types and fixed cost types are independently distributed 30

Finally, the third condition imposes mild restrictions on the effort cost function $h$ and the implied labor supply responses at the intensive margin.

Condition 3. There are two numbers $\mu_{1} \in(0, \infty)$ and $\mu_{2} \in(0, \infty)$ such that, for all $y>0$ and $\omega>\omega_{1}$, the effort cost function $h$ satisfies

(i) $\frac{1}{y} \frac{h_{y}(y, \omega)}{h_{y y}(y, \omega)} \leq \mu_{1}$, and

(ii) $-\frac{\omega}{y} \frac{h_{y \omega}(y, \omega)}{h_{y y}(y, \omega)} \geq \mu_{2}$.

Condition 3 imposes weak bounds on the elasticities of an agent's individually optimal income $y_{T}^{*}(\omega)$ with respect to the retention rate and his skill type. By part (ii), the elasticity of income with respect to the retention rate $1-T^{\prime}(y)$ is bounded from above by some finite number $\mu_{1}$ for all skill types. By part (ii), the elasticity of income with respect to the agent's skill level $\omega$ is bounded from below by some positive number $\mu_{2}$ for all skill types. Hence, Condition 3 can be regarded as a minimal regularity condition on the effort cost function $h$. It is satisfied for all commonly used functional forms, including the class of functions given by $h(y, \omega)=\frac{1}{1+1 / \sigma}\left(\frac{y}{\omega}\right)^{1+1 / \sigma}$ for any parameter value $\sigma \in(0, \infty){ }^{31}$

\section{Results}

In the following, I present the formal results of this paper and briefly describe their implications for the optimal design of income tax schedules. I start by investigating a relaxed version of the optimal tax problem, for which I derive three preliminary

\footnotetext{
${ }^{30}$ In Appendix B.4. I provide a relaxed version of Condition 2 for which all results of this paper continue to hold. I show that this relaxed version is satisfied if the conditional fixed cost distributions $\left(G_{j}\right)_{j=1}^{n}$ are given by Pareto, log-normal or normal distributions and if $G_{j}$ weakly dominates $G_{j+1}$ in the sense of first-order stochastic dominance for any $j \in J /\{n\}$.

${ }^{31}$ If $h$ is given by a function of this class, both elasticities are constant and given by $\varepsilon_{y, 1-T^{\prime}}(y, w)=\sigma$ and $\varepsilon_{y, \omega}(y, w)=1+\sigma$, respectively, for all $y>0$ and $\omega>0$.
} 
results of crucial importance. Then, I proceed by characterizing the labor supply distortions at both margins in the solution to the non-relaxed problem for three subsets of social weights in $\mathcal{A}$. As explained in Subsection 3.3, these results can easily be translated into results on the signs of the optimal marginal taxes and the optimal participation taxes. The economic mechanism behind these results will be explained in the following section. All formal proofs can be found in Appendix A.

\subsection{Preliminary results}

As Jacquet et al. (2013), I start by studying a relaxed version of the optimal tax problem that ignores the incentive compatibility (IC) constraints between all pairs of working agents $k$ and $l$ with different skill types, $\omega^{k} \neq \omega^{l}$. More precisely, I study the problem of maximizing social welfare (9) subject to the feasibility constraint (4) and the subset of IC constraints, first, between all agents with identical skill types,

$$
u(c(\omega, \delta), y(\omega, \delta) ; \omega, \delta) \geq u\left(c\left(\omega, \delta^{\prime}\right), y\left(\omega, \delta^{\prime}\right) ; \omega, \delta\right)
$$

for all $\omega \in \Omega$ and $\delta, \delta^{\prime} \in \Delta$, and second, between all unemployed agents,

$$
c(\omega, \delta) \geq c\left(\omega^{\prime}, \delta^{\prime}\right)
$$

for all $(\omega, \delta)$ and $\left(\omega^{\prime}, \delta^{\prime}\right)$ in $\Omega \times \Delta$ such that $y(\omega, \delta)=y\left(\omega^{\prime}, \delta^{\prime}\right)=0$. I denote by $\left(c^{\alpha R}, y^{\alpha R}\right)=\left(c_{j}^{\alpha R}, y_{j}^{\alpha R}\right)_{j=0}^{n}$ the solution to the relaxed problem for the social weight sequence $\alpha=\left(\alpha_{0}, \alpha_{1}, \ldots, \alpha_{n}\right)$.

Lemma 1 continues to apply to the set of allocations satisfying this reduced set of IC constraints. Hence, the solution to the relaxed problem involves, first, pooling by all unemployed agents and, second, pooling by all working agents with the same skill type. Moreover, an agent with skill type $\omega_{j}$ provides positive output if and only if his fixed cost type is below the skill-specific threshold $\delta_{j}$. I will say that $\left(c^{\alpha R}, y^{\alpha R}\right)$ is an interior solution if $\delta_{j} \in(\underline{\delta}, \bar{\delta})$ for all skill groups $j \in J$.

Lemma 3. There is a number $\chi \in(1,2]$ such that the relaxed problem has a unique and interior solution if $\alpha_{j} \in[0, \chi)$ for all $j \in J$. In this case, $y^{\alpha R}$ is undistorted at the intensive margin in all skill groups $j \in J$. At the extensive margin, $y^{\alpha R}$ is

(i) downwards distorted in skill group $j \in J$ if and only if $\alpha_{j}<1$, and

(ii) upwards distorted in skill group $j \in J$ if and only if $\alpha_{j}>1$.

The first sentence of Lemma3 3 indicates that the relaxed problem is well-behaved if the social weights of all groups of workers are below some threshold $\chi \in(1,2]$. 
For higher social weights, in contrast, the welfare function may either possess no interior maximum or multiple local extrema. In the latter case, an allocation that satisfies the first-order condition may violate the second-order condition. ${ }^{32}$ This is an inherent and well-known problem for models with labor supply responses at the extensive margin. For example, Choné \& Laroque (2011) discuss the problem of a potentially violated second-order condition for the purely extensive model of optimal taxation. As a result, the solution of the optimal tax problem may involve technical complications that are not directly related to the research question of this paper.

Hence, I proceed by restricting my attention to a subset of social weight sequences for which the existence of a well-behaved solution is ensured. In particular, I define the set $\mathcal{A}^{\chi} \subset \mathcal{A}$ as the subset of strictly decreasing weight sequences for which each element $\alpha_{j}$ is below the threshold $\chi$. All results in the remainder of this paper will be derived for weight sequences in $\mathcal{A}^{\chi}$.

The second and third sentence of Lemma 3 characterize the labor supply distortions in the solution to the relaxed problem, $\left(c^{\alpha R}, y^{\alpha R}\right)$. At the intensive margin, optimal output $y^{\alpha R}$ is undistorted in all skill groups. This may not come as a surprise to the reader. In intensive-margin models à la Mirrlees (1971), labor supply distortions at the intensive margin are necessary to relax the binding (downwards) IC constraints between adjacent skill types. In the relaxed problem considered here, however, these IC constraints are not taken into account anyway.

At the extensive margin, in contrast, optimal output $y^{\alpha R}$ may in general be distorted in both directions. In particular, labor supply is upwards distorted at the extensive margin in each skill group with a social weight above the population average 1, and downwards distorted in each skill group with a weight below 1 .

Hence, strictly decreasing social weights can give rise to two possible cases. First, if the social planner cares much more strongly about the unemployed than about the lowest-skilled workers, the social weight $\alpha_{1}$ of the latter group is below 1 . Then, labor supply is downwards distorted at the extensive margin in all skill groups. Second, if the social planner cares almost as much for the lowest-skilled workers as for the unemployed, the social weight $\alpha_{j}$ of the workers in the lowest $k \geq 1$ skill groups is above 1. Then, labor supply is upwards distorted in the lowest $k$ skill groups, and downwards distorted in all higher skill groups.

The crucial questions to be studied in the following are whether the introduction of the previously omitted IC constraints between workers with different skill types, first, leads to downwards, upwards or no distortions at the intensive margin, and second, changes this simple pattern of distortions at the extensive margin. I proceed

\footnotetext{
${ }^{32}$ Formally, the Lagrangian can become strictly convex in $c_{j}$ for $\alpha_{j}>\chi$.
} 
by investigating whether the solution to the relaxed problem, $\left(c^{\alpha R}, y^{\alpha R}\right)$, satisfies the downward IC constraint,

$$
c_{j+1}^{\alpha R}-h\left(y_{j+1}^{\alpha R}, \omega_{j+1}\right) \geq c_{j}^{\alpha R}-h\left(y_{j}^{\alpha R}, \omega_{j+1}\right)
$$

and the upward IC constraint,

$$
c_{j}^{\alpha R}-h\left(y_{j}^{\alpha R}, \omega_{j}\right) \geq c_{j+1}^{\alpha R}-h\left(y_{j+1}^{\alpha R}, \omega_{j}\right)
$$

for a single pair of adjacent skill types $\omega_{j}$ and $\omega_{j+1}$. Recall that, in an intensivemargin model à la Mirrlees (1971), the downward IC constraint is violated whenever the social planner cares more for the lower-skilled group, $\alpha_{j}>\alpha_{j+1}$. In the present model with labor supply responses at both margins, the answer to this question is more subtle.

Lemma 4. For any $j \in J_{-n}$ and $\alpha_{j} \in[0, \chi)$, the relaxed problem's solution violates

(i) the downward IC constraint if and only if

$$
\frac{\alpha_{j+1}-1}{\eta_{j+1}\left(c^{\alpha R}, y^{\alpha R}\right)}<\frac{\alpha_{j}-1}{\eta_{j}\left(c^{\alpha R}, y^{\alpha R}\right)}-\int_{y_{j}^{\alpha R}}^{y_{j+1}^{\alpha R}}\left[1-h_{y}\left(y, \omega_{j+1}\right)\right] d y
$$

(ii) the upward IC constraint if and only if

$$
\frac{\alpha_{j+1}-1}{\eta_{j+1}\left(c^{\alpha R}, y^{\alpha R}\right)}>\frac{\alpha_{j}-1}{\eta_{j}\left(c^{\alpha R}, y^{\alpha R}\right)}+\int_{y_{j}^{\alpha R}}^{y_{j+1}^{\alpha R}}\left[h_{y}\left(y, \omega_{j}\right)-1\right] d y \text {. }
$$

Lemma 4 provides two conditions that allow to determine whether the solution to the relaxed problem satisfies or violates the local IC constraints ${ }^{33}$ In particular, which IC constraint is violated depends mainly on two statistics that are familiar from previous optimal tax papers: the social weights $\alpha_{j}$ and $\alpha_{j+1}$ and the semielasticities of participation, $\eta_{j}$ and $\eta_{j+1}$. First, the downward IC is more likely to be violated if the concern for local redistribution is stronger, i.e., the difference $\alpha_{j}-\alpha_{j+1}$ is larger. Second, the satisfaction of each IC constraint depends on the difference $\eta_{j}-\eta_{j+1}$, which is strictly positive by Lemma 2 . The effect of an increase in this difference is not monotonic, however. If the weights $\alpha_{j}$ and $\alpha_{j+1}$ are below 1 , a larger difference $\eta_{j}-\eta_{j+1}$ implies that the downward IC is more likely to be

\footnotetext{
${ }^{33}$ Note that Jacquet et al. (2013) provide a similar condition for the model with a continuous skill set. Conditions (18) and (19) mainly differ from the one in Jacquet et al. (2013) by the integral terms in (19), which are strictly positive for any $\omega_{j+1} / \omega_{j}>1$ (and strictly increasing in $\omega_{j+1}$ given any $\left.\omega_{j}\right)$.
} 
violated. If $\alpha_{j}$ and $\alpha_{j+1}$ instead exceed 1 , a larger difference $\eta_{j}-\eta_{j+1}$ implies that the upward IC is more likely to be violated ${ }^{34}$

The conditions (18) and (19) also provide preliminary insights about the labor supply distortions in the optimal allocation. As shown in Jacquet et al. (2013), labor supply is downwards distorted at the intensive margin in all skill groups (below the very top) if the solution of the relaxed problem violates all local downwards IC constraints, i.e., if condition 18 is satisfied for all $j \in J_{-n}$. Put differently, upward distortions at the intensive margin can only be optimal if the relaxed problem's solution violates the upward IC constraint for some pairs of adjacent skills groups $j$ and $j+1$. In the following, I investigate in which cases the violation of an upward IC constraint is compatible with decreasing social weights, i.e., $\alpha_{j}>\alpha_{j+1}$.

For this purpose, I exploit that equation (19) implicitly defines a function $\beta_{j}^{U}$ : $[0, \chi) \rightarrow \mathbb{R}$ such that the upward IC is violated if and only if $\alpha_{j+1}>\beta_{j}^{D}\left(\alpha_{j}\right)$. Accordingly, equation (18) implicitly defines a function $\beta_{j}^{D}:[0, \chi) \rightarrow \mathbb{R}$ such that the downward IC is violated if and only if $\alpha_{j+1}<\beta_{j}^{D}\left(\alpha_{j}\right)$. Both functions cannot be expressed in closed form because the semi-elasticities $\eta_{j}$ and $\eta_{j+1}$ are endogenous quantities, which depend on the allocation $\left(c^{\alpha R}, y^{\alpha R}\right)$ and, ultimately, on the social weights $\alpha_{j}$ and $\alpha_{j+1}$. I can however study whether $\beta_{j}^{D}\left(\alpha_{j}\right)$ and $\beta_{j}^{U}\left(\alpha_{j}\right)$ can be located below $\alpha_{j}$, so that the solution to the relaxed problem satisfies the downward IC and violates the upward IC constraint given some decreasing social weights.

Lemma 5. For each $j \in J_{-n}$, the functions $\beta_{j}^{D}$ and $\beta_{j}^{U}$ are continuously differentiable, strictly increasing and satisfy $0<\beta_{j}^{D}(x)<\beta_{j}^{U}(x)<\chi$ for any $x \in[0, \chi)$. For each $j \in J_{-n}$, moreover, there is a number $a_{j}>1$ such that, if $\frac{\omega_{j+1}}{\omega_{j}} \in\left(1, a_{j}\right)$,

(a) $\beta_{j}^{D}(x)<x$ if and only if $x$ is above a unique number $\underline{\beta}_{j} \in(0,1)$, and

(b) $\beta_{j}^{U}(x)<x$ if and only if $x$ is above a unique number $\bar{\beta}_{j} \in(1, \chi)$.

Lemma 5 determines whether the relaxed problem's solution, first, violates the downward IC constraint, second, satisfies both IC constraints or, third, violates the upward IC constraint between the workers in skill groups $j$ and $j+1$, depending only on the social weights $\alpha_{j}$ and $\alpha_{j+1}$. In particular, all three cases arise for some combination with $\alpha_{j}<\chi$ and $\alpha_{j+1} \in\left(0, \alpha_{j}\right)$ if the relative distance between the skill levels $\omega_{j}$ and $\omega_{j+1}$ is sufficiently small ${ }^{35}$

Figure 1 illustrates the formal statements in Lemma 5 to make them more easily accessible. The shaded area below the $45^{\circ}$ line contains all possible combinations of

\footnotetext{
${ }^{34}$ Besides, the larger the relative distance between the skill types $\omega_{j}$ and $\omega_{j+1}$ is, the more likely are both IC constraints to be satisfied.

${ }^{35}$ If the relative distance between $\omega_{j}$ and $\omega_{j+1}$ exceeds the bound $a_{j}$, the solution of the relaxed problem may satisfy both local IC constraints (or one of them) for all $\alpha_{j}$ and $\alpha_{j+1}$ in $(0, \chi)$.
} 
the social weights $\alpha_{j}$ and $\alpha_{j+1}<\alpha_{j}$ in the relevant set $\mathcal{A}^{\chi}$. Additionally, Figure 1 includes two ascending graphs corresponding to the functions $\beta_{j}^{D}$ and $\beta_{j}^{U}$ in Lemma 5. Each graph crosses the $45^{\circ}$ line exactly once, i.e., each function has a unique fixed point. The fixed point $\underline{\beta}_{j}$ of function $\beta_{j}^{D}$ is located between 0 and the average weight 1 . The fixed point $\bar{\beta}_{j}$ of function $\beta_{j}^{U}$ is located between the average weight 1 and the upper threshold $\chi$. By Lemma 5 , the functions $\beta_{j}^{D}$ and $\beta_{j}^{U}$ partition the relevant set of weights $\mathcal{A}$ into three regions.

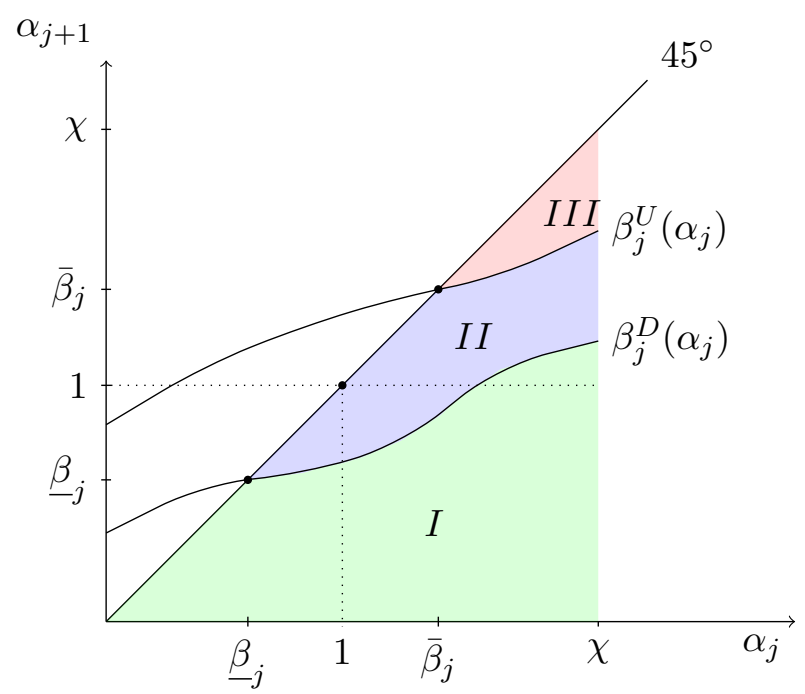

Figure 1: Local IC constraints in the relaxed problem's solution

For each tuple $\left(\alpha_{j}, \alpha_{j+1}\right)$ in region I, the relaxed problem's solution violates the downward IC constraint, i.e., higher-skilled workers consider their bundle $\left(c_{j+1}^{\alpha R}, y_{j+1}^{\alpha R}\right)$ less attractive than the bundle of the lower-skilled workers, $\left(c_{j}, y_{j}\right)$. As can be seen from Figure 1, this constellation results if either both social weights are low (below $\underline{\beta})$ or if the difference between the social weights $\alpha_{j}$ and $\alpha_{j+1}$ is large. In the first case, the planner has a strong desire to redistribute resources from the workers in these two skill groups to lower-skilled workers and/or unemployed agents. In the second case, the social planner has a strong desire to redistribute resources from the workers in the higher skill group $j+1$ to the workers in the lower skill group $j$.

For tuples $\left(\alpha_{j}, \alpha_{j+1}\right)$ in region II, the relaxed problem's solution satisfies both local IC constraints, i.e., the workers in each skill group prefer their own bundle to the one designated for the other group. As can be seen from Figure 1, this constellation mainly occurs if both social weights are close to 1 . In this case, the social planner has only a limited desire to redistribute resources between both groups of workers and the average agent in the economy.

For tuples $\left(\alpha_{j}, \alpha_{j+1}\right)$ in region III, the relaxed problem's solution violates the upward IC constraint, i.e., lower-skilled workers consider the bundle $\left(c_{j+1}^{\alpha R}, y_{j+1}^{\alpha R}\right)$ more 
attractive than their own bundle. Figure 1 shows that this constellation occurs if both social weights are above the average weight 1 and close enough to each other. Hence, the social planner has a strong concern for redistribution from higher-skilled workers to the workers in the skill groups $j$ and $j+1$, but only a limited desire to redistribute resources between the workers in these two skill groups. By Lemma 3 , labor supply in both skill groups is upwards distorted at the extensive margin in this case. As will become clear, this is no coincidence: the social planner can only prefer an allocation that violates the upward IC constraint if labor supply in both skill groups is upwards distorted at the extensive margin.

Summarizing, Lemma 5 clarifies that the relaxed problem's solution may indeed conflict with downward incentive-compatibility, as one might expect. But it may also conflict with upward incentive compatibility even if the social weight sequence is strictly decreasing, as it is the case for all weights in $\mathcal{A}^{\chi}$. Does this imply that, in the optimal allocation, upward IC constraints are binding and labor supply is upwards distorted at the intensive margin? For case III, this conjecture would be easy to confirm if the social planner would only take into account the upward IC constraint between the workers in skill groups $j$ and $j+1$. The optimal (secondbest) allocation must satisfy the full set of local IC constraints, however, potentially including a large number of other (downward and upwards) IC constraints that were violated by $\left(c^{\alpha R}, y^{\alpha R}\right)$. The main results below identify social weight sequences for which the distortions at the intensive margin can nevertheless be determined.

\subsection{Main results}

In the following, I characterize the labor supply distortions in the optimal allocations for decreasing social weight sequences. The first Proposition identifies three common properties of the optimal allocations for all weights in the set $\mathcal{A}^{\chi}$.

Proposition 1. For each $\alpha \in \mathcal{A}^{\chi}$, the optimal tax problem has a maximum $\left(c^{\alpha}, y^{\alpha}\right)$ with $\delta_{j} \in[\underline{\delta}, \bar{\delta})$ for all $j \in J$. In this solution,

(i) the consumption level $c_{0}^{\alpha}$ of the unemployed is strictly positive;

(ii) there is a number $k^{\alpha} \in(0, n)$ such that optimal output is

a) upwards distorted at the extensive margin in skill group $j$ if and only if $j \leq k^{\alpha}$, and

b) downwards distorted or undistorted at the intensive margin in skill group $j$ if $j>k^{\alpha}$; 
(iii) optimal output in the highest skill group $n$ is undistorted at the intensive margin and downwards distorted at the extensive margin.

By Proposition 1, the optimal tax problem has a well-defined solution with the following properties for any $\alpha \in \mathcal{A}^{\chi}$. First, the social planner provides a strictly positive transfer to the unemployed agents. Second, upward distortions at the extensive margin can only be optimal in the lowest skill groups with $j \leq k^{\alpha} 36$ Moreover, upward distortions at the intensive margin can only be optimal in a subset of these low-skill groups. Hence, upward distortions at the extensive margin are a necessary, but not sufficient condition for the optimality of upward distortions at the intensive margin. Third, Proposition 1 qualifies the classical no distortion at the top result. At the intensive margin, labor supply by the most productive workers is always undistorted as in Mirrlees (1971). At the extensive margin, in contrast, labor supply in the top skill group is always downwards distorted.

Apart from these common properties, there are substantial differences between the optimal allocations for alternative social weights in $\mathcal{A}^{\chi}$. In the interest of readability, I focus on three mutually exclusive subsets of social weights in $\mathcal{A}^{\chi}$. The definitions of these subsets make use of the functions $\beta_{j}^{D}$ and $\beta_{j}^{U}$ for all $j \in J_{-n}$, as identified in Lemma 5 .

Definition 1. The sets of weight sequences $\mathcal{A}^{N}, \mathcal{A}^{D}$ and $\mathcal{A}^{U}$ are defined as follows:

(i) Set $\mathcal{A}^{D}$ contains all sequences $\alpha \in \mathcal{A}^{\chi}$ such that $\alpha_{j+1} \leq \beta_{j}^{D}\left(\alpha_{j}\right)$ for all $j \in J_{-n}$, with a strict inequality for at least one $j \in J_{-n}$.

(ii) Set $\mathcal{A}^{N}$ contains all sequences $\alpha \in \mathcal{A}^{\chi}$ such that $\alpha_{j+1} \in\left[\beta_{j}^{D}\left(\alpha_{j}\right), \beta_{j}^{U}\left(\alpha_{j}\right)\right]$ for all $j \in J_{-n}$.

(iii) Set $\mathcal{A}^{U}$ contains all sequences $\alpha \in \mathcal{A}^{\chi}$ such that $\alpha_{2}>\beta_{1}^{U}\left(\alpha_{1}\right)$ and $\alpha_{j+1} \geq$ $\beta_{j}^{D}\left(\alpha_{j}\right)$ for all $j \in\{2, \ldots, n-1\}$.

The construction of each subset can be illustrated using Figure 1 above. In this figure, any weight sequence $\alpha \in \mathcal{A}^{\chi}$ can be depicted as an ascending scatter plot consisting of $n-1$ points, representing the weight-pairs $\left(\alpha_{j}, \alpha_{j+1}\right)$ for all $j \in J_{-n}$. To simplify the exposition, assume that the functions $\beta_{j}^{D}$ and $\beta_{j}^{U}$ were identical for all $j \in J_{-n}{ }^{37}$ Then, the social weights in the three subsets $\mathcal{A}^{N}, \mathcal{A}^{D}$ and $\mathcal{A}^{U}$ have the following properties.

\footnotetext{
${ }^{36} \mathrm{It}$ is also possible that labor supply in all skill groups is downwards distorted at the extensive margin (i.e., that $k^{\alpha}<1$ ).

${ }^{37}$ Note that this simplifying assumption is not imposed to define the subsets $\mathcal{A}^{D}, \mathcal{A}^{N}$ and $\mathcal{A}^{U}$, but only to explain their construction.
} 
For social weights in the first subset $\mathcal{A}^{D}$, all weight-pairs are located in region $I$ at the lowest part of Figure 1. As argued above, this represents a case in which the social planner has large concerns for local redistribution between all pairs of workers with adjacent skill types, including the workers with the lowest skill types. A limit case is given by the Rawlsian welfare function, which associated a positive weight to the unemployed and weights of zero to all groups of workers.

For social weights in the second subset $\mathcal{A}^{N}$, all weight-pairs are located in region $I I$ in the centre of Figure 1. In this case, the social planner has rather limited concerns for redistribution between all groups of workers.

For social weights in the third subset $\mathcal{A}^{U}$, the first weight-pair $\left(\alpha_{1}, \alpha_{2}\right)$ is located in region $I I I$ in the upper right part of Figure 1, while each of the remaining weight-pairs can be located in region $I I I$ or in the intermediate region $I I$. This case represents a social planner with a pronounced concern for redistribution from highly skilled workers to the workers in the lowest skill groups and the unemployed, but only a limited concern for redistribution among the workers in the lowest skill groups. An interesting limit case of this set involves identical weights above 1 associated to the unemployed and the workers in the $k \geq 2$ lowest skill groups.

I proceed by characterizing the optimal labor supply distortions for social weights in each of these three subsets, starting with the set $\mathcal{A}^{D}$. After that, Proposition 5 in the main text and Propositions 8 and 9 in Appendix B.6 clarify that social weights in all three sets can indeed arise for welfare functions with standard properties.

Proposition 2. For any $\alpha \in \mathcal{A}^{D}$, optimal output $y^{\alpha}$ is

- downwards distorted at the intensive margin in all skill groups $j \in J_{-n}$,

- downwards distorted at the extensive margin in all skill groups if $\alpha_{1}$ is below some threshold $\gamma^{D}>1$.

By Proposition 2, optimal labor supply by all except the highest-skilled workers is downwards distorted at the intensive margin for any social weights in the set $\mathcal{A}^{D}$. At the extensive margin, labor supply in all skill groups is downwards distorted if $\alpha_{1}$, the weight of the lowest-skilled workers, is below some threshold $\gamma^{D}$. Note that the threshold $\gamma^{D}$ is strictly higher than in the solution to the relaxed problem characterized in Lemma 3 (where it is equal to 1). Hence, labor supply distortions at both margins are interdependent: The condition for optimal upward distortions at the extensive margin becomes harder to satisfy if labor supply is downwards distorted at the intensive margin.

For all social weights in $\mathcal{A}^{D}$, the "central result of optimal income taxation" (Hellwig 2007) remains hence valid: The optimal marginal tax is strictly positive 
for all income levels below the top income $y_{n}^{\alpha}$. If and only if $\alpha_{1}<\gamma^{D}$, the optimal participation taxes are strictly positive at all income levels as well: the optimal income tax is a Negative Income Tax.

Proposition 3. For any $\alpha \in \mathcal{A}^{N}$, optimal output $y^{\alpha}$ is

- undistorted at the intensive margin in all skill groups;

- upwards distorted at the extensive margin in each skill group $j$ such that $\alpha_{j}>1$.

By Proposition 3, optimal labor supply is undistorted at the intensive margin in all skill groups for any social weights in the set $\mathcal{A}^{N}$. The optimal distortions at the extensive margin in each skill group depend on whether the social weight associated to this group is below or above the average weight of 1 . Hence, the labor supply distortions in the solutions to the relaxed problem and the full problem of optimal taxation are identical (see Lemma 3).

Note that this does not imply identical tax levels for all agents 38 The optimal allocation can be decentralized by a piecewise horizontal tax schedule, however. This finding is already in stark contrast to the before-mentioned "central result of optimal tax theory". If and only if $\alpha_{1}>1$, the least-productive workers benefit from a negative participation tax.

Proposition 4. For any $\alpha \in \mathcal{A}^{U}$, optimal output $y^{\alpha}$ is

- upwards distorted at the intensive margin in skill group 2;

- upwards distorted at the extensive margin in skill groups 1 and 2.

By Proposition 4, the optimal allocation involves upward distortions at both margins for any social weights in the set $\mathcal{A}^{U}$. In particular, labor supply in the two lowest skill groups is upwards distorted at the extensive margin, and labor supply by all workers with skill type $\omega_{2}$ is upwards distorted at the intensive margin 39 For all social weight in $\mathcal{A}^{U}$, the optimal tax is hence given by an Earned Income Tax Credit with negative marginal taxes and negative participation taxes at low income levels.

Summing up, the previous three propositions have clarified that any sign of the optimal marginal tax as well as the optimal participation tax is compatible with strictly decreasing social weights. More precisely, I have shown that the properties

\footnotetext{
${ }^{38}$ In contrast, the tax schedule will always be increasing over some income range. Additionally, it may also be decreasing over some (low) income range.

${ }^{39}$ In Appendix B, I construct subsets of $\mathcal{A}^{U}$ for which upward distortions at the intensive margin are optimal in multiple skill groups.
} 
of the optimal income tax depend on the strength of society's concerns for, first, redistribution from the rich to the poor (from high-skilled workers to low-skill workers) and, second, redistribution among the poor (from the low-skilled workers to the very-low-skilled workers and the unemployed). Propositions 2 to 4 have expressed these conditions on the redistributive concerns in terms of the (average) welfare weights associated to the agents in the $n+1$ income groups.

It is important to recall, however, that these social weights are endogenous objects that depend both on the properties of the welfare function (9) and on the allocation that maximizes this welfare function given the joint type distribution $K$. Hence, the previous result is only relevant if there exist well-behaved welfare functions for which the endogenous weight sequence $\bar{\alpha}$ indeed belongs to the set $\mathcal{A}^{U}$. In the following, I focus on welfare functions that involve a transformation $\Psi: \mathbb{R} \rightarrow \mathbb{R}$ and a type-dependent weighting function $\gamma: \Omega \times \Delta \rightarrow \mathbb{R}_{+}$with standard properties. More precisely, I say that a combination of $\Psi$ and $\gamma$ is regular if (a) $\Psi$ is strictly increasing and weakly concave and (b) $\gamma$ is weakly decreasing in $\omega$ and weakly increasing in $\delta$.

Proposition 5. There are two numbers $a^{U}>1, m \geq 3$ and two vectors $\left(\phi_{j}\right)_{j=1}^{n}$, $\left(\delta_{j}^{\prime}\right)_{j=1}^{n}$ with $\phi_{j+1} \geq \phi_{j}$ for all $j \in J_{-n}, \phi_{j} \gtrless 1$ for $j \gtrless m$ and $\delta_{j}^{\prime} \in(\underline{\delta}, \bar{\delta})$ for all $j \in J$ such that, if

(a) $\frac{\omega_{2}}{\omega_{1}}<a^{U}$

(b) $n \geq m$ and

(c) $\sum_{j=1}^{n} f_{j} G_{j}\left(\delta_{j}^{\prime}\right) \phi_{j}>1$,

there exist regular combinations of $\Psi$ and $\gamma$ for which $\bar{\alpha} \in \mathcal{A}^{U}$.

Proposition 5 provides three conditions that jointly ensure the existence of wellbehaved welfare functions for which an Earned Income Tax Credit is optimal ${ }^{40}$ Although these conditions appear complicated, they can easily be interpreted. Condition (a) requires the relative distance between the two lowest skill types to be sufficiently small. The remaining two conditions ensure that there is a sufficiently large share of high-skilled workers in the economy. This requirement can be decomposed into two elements.

By Condition (b), the cardinality $n$ of the skill set $\Omega$ has to be equal to (or above) some finite threshold $m \geq 3$. Recall that the ratio $\omega_{j+1} / \omega_{j}$ is assumed to exceed

\footnotetext{
${ }^{40}$ Correspondingly, Propositions 8 and 9 in Appendix B.6 provide sufficient conditions for the existence of well-behaved welfare functions such that the welfare weights $\bar{\alpha}$ belong to the sets $\mathcal{A}^{D}$ and $\mathcal{A}^{N}$, respectively.
} 
$1+\varepsilon$ for some $\varepsilon>0$. Hence, Condition (b) requires the relative difference between the highest skill type $\omega_{n}$ and the lowest skill type $\omega_{1}$ to be large enough.

By Condition (c), the population share of the agents with high skill types $\left(\omega_{m}\right.$ or higher) and low fixed cost types has to be sufficiently large. To see this, note that the condition compares a weighted average over the increasing sequence $\phi=$ $\left(\phi_{1}, \phi_{2}, \ldots, \phi_{n}\right)$ with 1 . Each element $\phi_{j}$ is weighted by the population share of the agents with skill type $\omega_{j}$ and fixed cost types below some threshold $\delta_{j}^{\prime}$ (i.e., the agents in skill group $j$ with the largest preference for participating in the labor market). By construction, only the elements with index $m$ or higher in $\phi$ are larger than 1 . Hence, condition (c) is ensured to be satisfied if the population share $\sum_{j=m}^{n} f_{j} G_{j}\left(\tilde{\delta}_{j}\right)$ of highly productive agents is close to 1 , and to be violated if the same population share is close to zero.

While condition (a) can be perceived as a regularity condition, the two latter parts are economically more restrictive. A crucial aspect is given by the level of the threshold skill type $\omega_{m}$ (relative to the lowest skill type $\omega_{1}$ ). In general, the required ratio of $\omega_{m} / \omega_{1}$ depends in a non-trivial way on all primitives of the model, including the effort cost function $h$, the type set $\Omega \times \Delta$ and the type distribution $K$. To assess the restrictiveness of condition (b), I consider a numerical example with commonly used functional forms in Appendix C. For this example, the condition turns out to be reasonably mild: It is satisfied whenever the skill set is sufficiently "dense" and the ratio $\omega_{n} / \omega_{1}$ of the highest to the lowest skill type exceeds 2 .

\section{The underlying mechanism}

In the following section, I explain the economic mechanism behind the main results of this paper. The optimal pattern of labor supply distortions are driven by, first, the standard trade-off between equity and efficiency and, second, a previously neglected trade-off between labor supply distortions at both margins. I start by studying an auxiliary problem that helps to isolate the latter trade-off and clarify its implications for the optimal allocation. Then, I show how the properties of the optimal allocation are affected by the introduction of redistributive concerns. On the way, I also comment on crucial steps in the proofs of my results.

\subsection{The tradeoff between intensive and extensive efficiency}

In the following, I study the auxiliary problem to maximize efficiency subject to a reduced set of incentive compatibility constraints and to the constraint that some 
fixed amount of resources is redistributed from the high-skill workers to the unemployed agents and the low-skill workers. More formally, I analyze the problem to minimize the deadweight loss from labor supply distortions (6) over the set of feasible allocations, subject to the constraint that the exogenous amount $R>0$ of resources is transferred from the set of workers with skill type $\omega_{3}$ and higher to the set of unemployed agents and workers with skill types $\omega_{1}$ and $\omega_{2}$,

$$
\sum_{j=1}^{n} f_{j}\left[1-G_{j}\left(\delta_{j}\right)\right] c_{0}+\sum_{j=1}^{2} f_{j} G_{j}\left(\delta_{j}\right)\left(c_{j}-y_{j}\right)=\sum_{j=3}^{n} f_{j} G_{j}\left(\delta_{j}\right)\left(y_{j}-c_{j}\right)=R,
$$

and to the incentive compatibility constraints between all agents with identical skills (14), between all unemployed agents (15), and between the workers in the lowest two skill groups,

$$
\begin{aligned}
& c_{2}-h\left(y_{2}, \omega_{2}\right) \geq c_{1}-h\left(y_{1}, \omega_{2}\right), \\
& c_{1}-h\left(y_{1}, \omega_{1}\right) \geq c_{2}-h\left(y_{2}, \omega_{1}\right) .
\end{aligned}
$$

I henceforth refer to this program as the problem of efficient redistribution. I denote its solution by $\left(c^{E}, y^{E}\right)$ and the implied vector of participation thresholds by $\delta^{E}$. The following Lemma identifies the labor supply distortions in this solution.

Lemma 6. Consider a redistribution amount $R>0$ such that the solution $\left(c^{E}, y^{E}\right)$ to the efficient distribution problem exists and is interior.

(i) Output $y^{E}$ is upwards distorted at the extensive margin in skill groups 1 and 2.

(ii) There is a number $a^{E}>1$ such that, if $\frac{\omega_{2}}{\omega_{1}} \in\left(1, a^{E}\right)$, output $y^{E}$ is upwards distorted at the intensive margin in skill group 2.

By Lemma 6, redistributing resources in the most efficient way requires to distort labor supply of low-skill workers upwards at both margins. This result holds whenever, first, the distance between skill groups 1 and 2 is sufficiently small and, second, the problem has an interior solution. The first qualification is related to the assumption of a discrete skill set and will become clear below. The second qualification has to be made because the problem may fail to have a well-behaved solution for high levels of $R$. In particular, it may be impossible to collect the required amount of resources from the high-skilled workers due to Laffer curve effects. ${ }^{41}$ Besides, the solution for high levels of $R$ may involve labor market participation by all low-skill

\footnotetext{
${ }^{41}$ The more resources are transferred from high-skill workers to unemployed agents, the more high-skill workers become unemployed. Hence, the level of transfers is bounded from above.
} 
agents, i.e., a boundary solution with extreme upward distortions. As both problems have no relevance for the optimal income tax problem and the trade-off between intensive efficiency and extensive efficiency, I henceforth restrict my attention to cases with a well-behaved solution.

Upward distortions at the extensive margin. I start by explaining why efficient redistribution $y^{E}$ gives rise to upward distortions at the extensive margin in both low-skill groups, i.e., why the participation threshold $\delta_{j}^{E}$ exceeds its first-best level $\delta^{*}\left(\omega_{j}\right)$ for $j \in\{1,2\}$ (see first part of Lemma 6). Assume for a moment that the local IC constraints between the workers in both low-skill groups are not binding and can hence be ignored. In this case, the efficiency-maximizing allocation $\left(c^{E}, y^{E}\right)$ does not involve distortions at the intensive margin. Hence, the social planner only faces the problem to minimize the deadweight loss from distortions at the extensive margin.

For each $j \in\{1,2\}$, the optimal level of the participation threshold is implicitly defined by the first-order condition with respect to $c_{j}$,

$$
\delta_{j}^{E}-\delta^{*}\left(\omega_{j}\right)=c_{j}^{E}-y_{j}^{E}-c_{0}^{E}=\frac{\lambda_{E}}{1-\lambda_{E}} \frac{1}{\eta_{j}\left(c^{E}, y^{E}\right)}>0
$$

where $\lambda_{E}$ is the Lagrange multiplier associated with the constraint that $R$ resources have to be transferred to the unemployed and the working poor ${ }^{42}$ Equation (23) has two crucial implications.

First, low-skill labor supply is upwards distorted at the extensive margin whenever the redistribution constraint is binding, i.e., the amount $R$ is strictly positive. Put differently, efficient redistribution always involves larger transfers to the lowskilled workers than to the unemployed. To provide the economic intuition behind this result, consider an initial allocation in which labor supply in both low-skill groups is undistorted at both margins. This requires that, first, the output levels $y_{1}$ and $y_{2}$ satisfy $h_{y}\left(y_{1}, \omega_{1}\right)=h_{y}\left(y_{2}, \omega_{2}\right)=1$ and, second, identical transfers are provided to the low-skill workers and the unemployed agents, $c_{1}-y_{1}=c_{2}-y_{2}=c_{0}>0$. Feasibility requires that these transfers are paid by the high-skill workers, i.e., $y_{j}-c_{j}>0$ for all $j \geq 3$. Hence, labor supply in the high-skill groups must be downwards distorted at the extensive margin.

Assume now that the planner reduces the consumption level $c_{0}$ of the unemployed and increases the consumption levels $c_{1}$ and $c_{2}$ of the working poor in a budgetbalancing way, holding $R$ constant. This has two effects on labor supply. First, some

\footnotetext{
${ }^{42}$ Note that $\lambda_{E}$ takes a value in the interval $(0,1)$ for any $R>0$ such that $\left(c^{E}, y^{E}\right)$ is interior. Below, I show how the levels of $\delta_{1}^{E}$ and $\delta_{2}^{E}$ are affected by the presence of the IC constraints.
} 
previously unemployed agents in both low-skill groups start working, creating an upwards distortion at the extensive margin. Initially, this only leads to a negligible (second-order) increase in the deadweight loss, because labor supply in these groups was undistorted before. Second, some previously unemployed agents in all high-skill groups start working due to the reduction in $c_{0}$. This response leads to a first-order reduction in the deadweight loss, because labor supply was downwards distorted at the extensive margin before and is less so now. Hence, providing larger transfers to the low-skilled workers than to the unemployed increases extensive efficiency, although it leads to upward distortions at the extensive margin.

Second, the first-order conditions with respect to $c_{1}$ and $c_{2}$ imply that the workers in both low-skill groups receive different transfers. It is worth noting that, if the local IC constraints between both low-skill groups are ignored, the problem of efficient redistribution is structurally identical to the Ramsey problem of optimal commodity taxation (or optimal linear pricing). Accordingly, equation (23) represents an inverse elasticity rule: The transfer to skill group $j \in\{1,2\}$ has to be inversely related to the semi-elasticity $\eta_{j}$ of participation in this skill group ${ }^{43}$ Recall that the relative sizes of participation responses are pinned down by Condition 1: $\eta_{1}$ exceeds $\eta_{2}$ in every implementable allocation (see Lemma 2). For any $R>0$, the efficient-maximizing allocation must hence involve strictly higher transfers to the higher-skilled workers than to the lowest-skilled workers, $c_{2}^{E}-y_{2}^{E}>c_{1}^{E}-y_{1}^{E}$.

Upward distortions at the intensive margin. By the previous paragraph, the solution to the problem of efficient redistribution involves higher transfers to the workers in skill group 2 than to the less skilled workers in group 1. This gives rise to the question whether the allocation defined by equation (23) and the redistribution constraint $(20)$ violates the upward IC constraint ${ }^{44}$ As Lemma 6 indicates, the answer to this question is positive whenever the skill set is sufficiently "dense", i.e., the distance between skill levels $\omega_{1}$ and $\omega_{2}$ is sufficiently small.

Note that the formal derivation of this crucial result involves a non-standard complication. In particular, as I have not imposed any functional form assumptions on the effort cost function $h$ and the joint type distribution $K$, the violation of the upward IC constraint cannot be verified directly for specific skill distances. The formal proof resolves this problem by studying how the participation threshold $\delta_{2}^{E}$ is affected by variations in the skill level $\omega_{2}$, given some fixed level of $R$. In particular, I first use equation $(23)$ to investigate the optimal relation between $\delta_{1}^{E}$ and $\delta_{2}^{E}$ for the

\footnotetext{
${ }^{43}$ Note that the classical inverse elasticity rule is expressed in terms of standard elasticities instead of semi-elasticities.

${ }^{44} \mathrm{By}$ the previous arguments, the downward IC constraint is trivially satisfied for any $R \geq 0$.
} 
limit case where $\omega_{2}$ equals $\omega_{1}$. Second, I show that allocation $\left(c^{E}, y^{E}\right)$ unambiguously violates the upward IC constraint after a marginal increase in $\omega_{2}$ as long as Condition 1 is satisfied.

The previous arguments have clarified that the upward IC constraint is binding in $\left(c^{E}, y^{E}\right)$ if the distance between $\omega_{1}$ and $\omega_{2}$ is small enough. Assume that this condition is met. In this case, the social planner cannot at the same time set the transfers to both groups of low-skill workers according to the inverse elasticity rule 23) and avoid distortions at the intensive margin. Specifically, to satisfy the inverse elasticity rule, he has to relax the upward IC constraint by distorting labor supply $y_{2}$ upwards at the intensive margin. Put differently, the planner can only increase extensive efficiency if he reduces intensive efficiency and vice versa. This trade-off constitutes a crucial difference between the problem of efficient redistribution and the standard Ramsey problem.

To minimize the overall deadweight loss (6), the planner has to implement the allocation that equates the marginal deadweight losses from distortions at both margins, representing the optimal compromise between intensive efficiency and extensive efficiency. Formally, the efficiency-maximizing allocation has to satisfy

$$
\begin{aligned}
\frac{f_{2} G_{2}\left(\delta_{2}^{E}\right)\left[h_{y}\left(y_{2}^{E}, \omega_{2}\right)-1\right]}{h_{y}\left(y_{2}^{E}, \omega_{1}\right)-h_{y}\left(y_{2}^{E}, \omega_{2}\right)} & =\Lambda\left\{\eta_{1}\left[\delta_{1}^{E}-\delta^{*}\left(\omega_{1}\right)\right]-\eta_{2}\left[\delta_{2}^{E}-y_{2}^{E}+h\left(y_{2}^{E}, \omega_{2}\right)\right]\right\} \\
& >0
\end{aligned}
$$

where $\Lambda:=\left[f_{1} f_{2} G_{1}\left(\delta_{1}^{E}\right) G_{2}\left(\delta_{2}^{E}\right)\right] /\left[f_{1} G_{1}\left(\delta_{1}^{E}\right)+f_{2} G_{2}\left(\delta_{2}^{E}\right)\right]$.

For the interpretation of this condition, consider a marginal increase in $y_{2}$, which relaxes the upward IC constraint and hence allows to raise the difference between the transfers to the workers in skill groups 1 and 2. The left-hand side of equation 24. captures the induced increase in the intensive deadweight loss. In particular, the term in the numerator states the difference between the marginal rate of substitution $h_{y}\left(y_{2}, \omega_{2}\right)$ and the marginal rate of transformation 1 , while the term in the denominator quantifies the extent to which the upward IC is relaxed.

The right-hand side (24) captures the reduction in the extensive deadweight loss that results from raising the difference between both transfers. In particular, the term in brackets evaluates how much the allocation $\left(c^{E}, y^{E}\right)$ deviates from the inverse elasticity rule (23). The larger this term is, the more beneficial it is to distort $y_{2}$ upwards in order to increase the difference between both transfers 45

Summing up, the solution to the auxiliary problem of efficient redistribution

\footnotetext{
${ }^{45}$ Note that equation (24) is also satisfied if the distance between both skill levels $\omega_{1}$ and $\omega_{2}$ is above the threshold $a_{E}$. In this case, allocation $\left(c^{E}, y^{E}\right)$ satisfies the inverse elasticity rule and involves no distortion at the intensive margin. Thus, both sides of the equation equal zero.
} 
involves upward distortions in labor supply at both margins. Note that these insights extend to a more general version of the efficient redistribution problem in which the planner wants to redistribute resources to the unemployed and the workers with the lowest $k \in(2, n)$ skill types (from all higher-skilled workers), and takes into account the local IC constraints between all workers with skill types $\omega_{1}$ to $\omega_{k}$. In this case, the efficiency-maximizing allocation involves upward distortions at the extensive margin in the skill groups 1 to $k$, and upward distortions at the intensive margin in the skill groups 2 to $k$. As shown in section 3.3, this allocation can be implemented by means of an EITC-type income tax schedule with negative participation taxes and negative marginal taxes for low-skill workers.

\subsection{The effects of redistributive concerns}

The following section sketches how the previous insights are affected by the introduction of redistributive concerns. For this purpose, I turn to the problem of maximizing social welfare (9) subject to the feasibility constraint (4) and the subset of incentive compatibility constraints (14), (15), (21) and (22), which were also included in the problem of efficient redistribution. ${ }^{46}$ The following paragraphs explain how changes in the social weights $\alpha_{0}, \alpha_{1}$ and $\alpha_{2}$ affect the properties of the optimal allocation. To simplify the exposition, I concentrate on social weight sequences that put zero weight on the workers in all higher-skill groups, $\alpha_{j}=0$ for all $j \in\{3, \ldots, n\}$.

As a benchmark, consider the case with identical weights for the unemployed and both types of low-skill workers, i.e., $\alpha_{0}=\alpha_{1}=\alpha_{2}>1$. In this case, the social planner has no concerns for redistribution between the agents in these three groups. Hence, the solution to this welfare maximization problem is identical to the solution of the efficient redistribution problem, given some strictly positive amount $R^{\prime}>0$ of redistribution. As shown above, this allocation involves upward distortions among the low-skilled workers at both margins.

\section{Concerns for redistribution from low-skill workers to unemployed agents.}

Assume that the planner associates a higher weight to the unemployed than to the low-skill workers, i.e., $\alpha_{0}>\alpha_{1}=\alpha_{2}=\alpha^{\prime}$. In this case, he has a concern for local redistribution from the low-skill workers to the unemployed, but no concern for redistribution between the low-skill workers. It turns out that the level of $\alpha^{\prime}$ affects the optimal distortions at both margins.

\footnotetext{
${ }^{46}$ In contrast to the relaxed problem in section 5 , the problem studied here also takes into account the local IC constraints (21) and (22) between the workers in skill groups 1 and 2 .
} 
First, these redistributive concerns call for giving higher transfers to the unemployed than to the working poor, inducing downward distortions at the extensive margin. As shown above, however, maximizing efficiency requires to provide higher transfers to the low-skilled workers, inducing upward distortions at the extensive margin. The strength of the redistributive concerns as measured by the difference $\alpha_{0}-\alpha^{\prime}$ determines which of these countervailing forces dominates. In particular, the optimal allocation involves upward distortions at the extensive margin in both skill groups if $\alpha^{\prime}$ is above the average weight 1 , but downward distortions if $\alpha^{\prime}$ is below 1 (see Lemma 3).

Second and less obviously, the relative size of $\alpha^{\prime}$ also affects the optimal distortions at the intensive margin. Because the planner cares equally for all low-skill workers, the optimal transfers for both low-skill groups are defined by a slightly adjusted version of the inverse elasticity rule (23) ${ }^{47}$ Hence, as long as low-skill labor supply is upwards distorted at the extensive margin (i.e., for $\alpha^{\prime}>1$ ), the optimal transfer to the workers in group 2 is larger than the one to the workers in group 1. If low-skill labor is downwards distorted at the extensive margin (i.e., for $\alpha^{\prime}<1$ ), in contrast, the inverse elasticity rule implies that the optimal transfer to the workers in group 2 is smaller (or more negative) than the one to the workers in group 1. Consequently, the optimal allocation can only involve a binding upward IC constraint between skill groups in which labor supply is upwards distorted at the extensive margin.

More precisely, Lemma 5 states that the upward IC is binding if and only if $\alpha^{\prime}$ exceeds a unique threshold $\bar{\beta}_{1}>1$ (see illustration in Figure 1). Note that this uniqueness result is only ensured if the difference between the optimal transfers to both skill groups is monotonically increasing in the social weight $\alpha^{\prime}$. Intuitively, this property seems reasonable: With higher social weight $\alpha^{\prime}$, the social planner provides larger transfers to both groups of workers. To limit the deadweight loss, the larger share of these additional transfers should go to the higher-skilled workers in group 2, who respond less elastically at the extensive margin.

This basic intuition does not take into account, however, that variations in the transfers to both low-skill groups may affect the relative sizes of the elasticities $\eta_{1}$ and $\eta_{2}$. In the proof of Lemma 5 , I show that the difference between both optimal transfers is nevertheless monotonic if the joint type distribution satisfies

$$
\left(2-\alpha^{\prime}\right)\left[A_{1}\left(\delta_{1}\right)-A_{2}\left(\delta_{2}\right)\right]>\left(1-\alpha^{\prime}\right)\left[a_{1}\left(\delta_{1}\right)-a_{2}\left(\delta_{2}\right)\right]
$$

\footnotetext{
${ }^{47}$ If the local IC constraints between both groups of low-skill workers can be ignored, the welfaremaximizing transfer to workers in skill group $j$ is defined by $\delta_{j}-\delta^{*}\left(\omega_{j}\right)=\left(\alpha^{\prime}-1\right) \frac{1}{\eta_{j}(c, y)}$.
} 
for all relevant combinations of $\delta_{1}$ and $\delta_{2} \in \Delta$, where $A_{k}\left(\delta_{k}\right)$ and $a_{k}\left(\delta_{k}\right)$ are the hazard rates of the $c d f$ and the $p d f$ of the fixed-cost distribution $G_{k}$ in skill group $k$. Condition 2 ensures that this inequality holds for all levels of $\alpha^{\prime} \in(0, \chi)$. Intuitively, it hence serves as a regularity condition that rules out drastic changes in the relative sizes of the semi-elasticities $\eta_{1}$ and $\eta_{2}$.

Concerns for redistribution among low-skill workers. Finally, assume that the social planner cares more for the lowest-skilled workers than for the slightly higher-skilled workers, $\alpha_{1}>\alpha_{2}$. These redistributive concerns call for providing higher transfers to the lower-skilled workers in group 1. As shown above, however, maximizing efficiency requires to provide higher transfers to the higher-skilled workers in group 2 as long as both social weights exceed 1. To balance both aspects, the transfers to the higher-skilled workers in group 2 have to be smaller than in the efficiency-maximizing allocation $\left(c^{E}, y^{E}\right)$. Consequently, there is less need (or no need at all) to relax the upward IC constraint through upward distortions at the intensive margin: the optimal output $y_{2}$ by workers with skill type $\omega_{2}$ is strictly smaller than $y_{2}^{E}$.

More precisely, labor supply in the higher-skilled group 2 continues to be upwards distorted at the intensive margin if the local redistributive concerns as measured by the difference $\alpha_{1}-\alpha_{2}$ are weak and both social weights are sufficiently high (see Lemma 5 and Figure 1). If the redistributive concerns are stronger, in contrast, the optimal allocation involves larger transfers to the lower-skilled group. In this case, the downward IC constraint is binding, and labor supply in the lower-skilled group 1 is downwards distorted at the intensive margin.

\subsection{Full incentive compatibility}

The previous sections have clarified why and when optimal labor supply may be upwards distorted at both margins. For this purpose, I have studied two auxiliary problems, in which I have ignored the IC constraints between the workers in all but the two lowest skill groups. The following section explains how these insights extend to the unrelaxed problem of optimal income taxation, where the complete set of incentive compatibility constraints is taken into account.

In the solution to this unrelaxed problem, the direction and magnitude of labor supply distortions in skill group $j$ do not only depend on the social weights associated to the workers in this group and in the two adjacent groups $j-1, j+1$, but on the complete sequence of social weights $\alpha=\left(\alpha_{0}, \alpha_{1}, \ldots, \alpha_{n}\right)$. Put differently, the IC constraints along the entire skill set $\Omega$ are interconnected and the set of binding IC 
constraints has to be determined jointly.

The implications of this interconnection can be clarified transparently for the problem of efficient redistribution, where a fixed amount of resources has to be transferred from the workers with skill type $\omega_{3}$ and higher to the workers with skill types $\omega_{1}$ and $\omega_{2}$ and the unemployed (see Section 6.1). If only the local IC constraints between the workers in the two lowest skill groups are taken into account, the solution to this problem involves a binding upward IC constraint and upward distortions in $y_{2}$. But this solution typically violates the downward IC constraint between the workers in skill groups 2 and the workers in skill group 3, because the social planner transfers resources from the latter to the former ${ }^{48}$ If the social planner has to take into account this constraint as well, he can only provide lower transfers to the workers in skill group 2 than in his preferred allocation $\left(c^{E}, y^{E}\right)$. Unless more specific assumptions are imposed, it is impossible to determine whether the solution to the unrelaxed problem involves a binding upward IC constraint or, in contrast, a binding downward IC constraint between skill groups 1 and 2 .

Propositions 2 to 4 identify social weight sequences for which the optimal direction of labor supply distortions can be uniquely determined despite these complications. In the following, I concentrate on social weight sequences for which the optimal allocation involves upward distortions at both margins. First, I explain why this pattern results for all weight sequences in the set $\mathcal{A}^{U}$ (Proposition 4). Second, I sketch why the existence of a sufficiently large share of high-skilled workers ensures that some well-behaved welfare functions indeed give rise to (endogenous) social weights in the set $\mathcal{A}^{U}$ (Proposition 5).

By Proposition 4, the optimal allocation involves upward distortions at both margins for any $\alpha \in \mathcal{A}^{U}$. The intuition behind this result can most easily be provided by an example with three skill groups only. Fix some social weight sequence $\alpha \in \mathcal{A}^{U}$. By the definition of set $\mathcal{A}^{U}$, the relaxed problem's solution violates the upward IC constraint between the workers in skill groups 1 and 2, and satisfies the downward IC between the workers in skill groups 2 and 3. Put differently, the social planner would prefer to provide higher transfers towards the workers in the middle group 2 than compatible with the upward IC constraint at the bottom. In the optimal allocation, this upward IC constraint is necessarily binding. However, the downward IC constraint between the skill groups 2 and 3 can be binding as well. Why is an upward distortion in output $y_{2}^{\alpha}$ ensured to be optimal in this case?

Assume that both the upward IC constraint at the bottom and the downward

\footnotetext{
${ }^{48}$ Additionally, it can be shown that allocation $\left(c^{E}, y^{E}\right)$ violates the downward IC constraints between the workers with all pairs of higher skill types if the ratio $\omega_{j+1} / \omega_{j}$ is sufficiently small.
} 
IC constraint at the top are binding in the optimal allocation, and that $y_{2}^{\alpha}$ is downwards distorted 49 Both binding constraints imply that the social planner has to make the workers in group 2 worse off than in his preferred allocation $\left(c^{\alpha R}, y^{\alpha R}\right)$. Hence, the bundle $\left(c_{2}^{\alpha}, y_{2}^{\alpha}\right)$ is less attractive to all other workers as well. Due to the single-crossing condition, the downward distortion in $y_{2}^{\alpha}$ makes this bundle even less attractive to high-skill workers. Hence, the high-skill workers strictly prefer their own bundle in the planner's preferred allocation $\left(c^{\alpha R}, y^{\alpha R}\right)$ to the bundle $\left(c_{2}^{\alpha}, y_{2}^{\alpha}\right)$, which gives a contradiction. Hence, either the downward IC constraint at the top cannot be binding, or $y_{2}^{\alpha}$ cannot be downwards distorted. Figure 7 in Appendix B illustrates these arguments graphically. Additionally, it clarifies that the downward IC constraint at the top can actually be binding in the optimal allocation, but can never overturn the upward distortion in $y_{2}^{\alpha}$.

Proposition 5 provides three jointly sufficient conditions for the existence of regular combinations of $\Psi$ and $\gamma$ such that the optimal allocation involves upward distortions at both margins. As explained in Section 5, the first condition requires the relative distance between the lowest skill types $\omega_{2}$ and $\omega_{1}$ to be sufficiently small. The second and third conditions jointly require the share of high-skill workers, i.e., agents with high skill types and low fixed cost types, to be sufficiently large.

The first condition (a) requires the relative distance between the skill types $\omega_{1}$ and $\omega_{2}$ to be sufficiently small. Its relevance is explained for the problem of efficient redistribution in Section 6.1 above. Whenever the social planner transfer more resources to the low-skilled workers than to the unemployed, efficiency considerations call for providing larger transfers to the more skilled workers in group 2 than to the less skilled workers in group 1 . If the distance between $\omega_{1}$ and $\omega_{2}$ is large, however, the upward IC constraint may remain slack despite these differential transfers. In this case, upward distortions at the intensive margin cannot provide any benefits. This insight directly extends to the solution of the optimal tax problem, where the social planner has redistributive concerns.

The remaining conditions (b) and (c) require the existence of a sufficiently large share of high-skill workers. Intuitively, their role is to ensure that a potentially optimal allocation with upward distortions at both margins is both feasible and incentive-compatible. Recall that, for any $\alpha \in \mathcal{A}^{U}$, the transfers to the low-skilled workers are larger than the strictly positive transfers to the unemployed agents. First, feasibility requires that these transfers are paid by the remaining agents, i.e., the workers with higher skill types. Hence, the group of agents with high

\footnotetext{
${ }^{49}$ Alternatively, either both upward IC constraints or only the upward IC constraint at the bottom could be binding. In both cases, $y_{2}^{\alpha}$ is upwards distorted by standard arguments.
} 
skill types and low fixed cost types must be sufficiently large. Second, incentive compatibility requires that the high-skill workers prefer to provide high output levels despite paying transfers instead of providing little output and receiving transfers, i.e., mimicking low-skill workers. This condition can only be satisfied if the highskill agents can at the same time pay substantial transfers and keep much higher consumption than the low-skill workers. Hence, the difference between the highest and the lowest skill types must be large enough 50 Moreover, the skill difference must also induce a large difference in the output levels $y_{1}$ and $y_{n}$, which is ensured by Condition 3 on the effort cost function $h$. If these conditions are met, the social planner can design a tax schedule $T$ that involves (a) large differences between the tax levels $T\left(y_{n}\right)$ and $T\left(y_{1}\right)$ and at the same time (b) small marginal taxes that induce only negligible labor supply distortions at the intensive margin.

From an applied perspective, Proposition 5 has a more general implication for the optimal design of income taxes. When the jointly sufficient conditions (a) to (c) are met, the optimal labor supply can also be upwards distorted at both margins in more than just one skill group, e.g., in the subset of all skill groups 2 to $k$. By the arguments provided above, however, this subset can only comprise a minority of the population, and only agents with skill types that are considerably below the top skill $\omega_{n}$. Hence, an Earned Income Tax Credit always has to be restricted to a limited set of agents with income levels that are well below the average income. Otherwise, it would not be possible to transfer resources from the high-skilled tax-payers to the low-skilled EITC recipients in a feasible and incentive-compatible way.

\section{Conclusion}

The paper has studied optimal income taxation in an empirically plausible model with labor supply responses at the intensive margin and at the extensive margin. Using a novel modeling strategy, it is the first paper to provide sufficient conditions for the optimality of an Earned Income Tax Credit with negative marginal taxes and negative participation taxes at low income levels. In particular, the optimal income tax is given by an EITC if society has strong concerns for redistribution from the rich to the poor, but only weak concerns for redistribution from the poor to the very poor. In contrast, the optimal income tax is given by a Negative Income Tax with positive marginal taxes and positive participation taxes at all income levels if society has strong concerns for redistribution from the rich to the poor as well as strong

\footnotetext{
${ }^{50}$ Given a discrete skill set in which the relative distance $\omega_{j+1} / \omega_{j}$ is bounded away from zero, any skill difference can be ensured by requiring the number of skill groups $n$ to be large enough.
} 
concerns for redistribution from the poor to the poorest. As shown above, these results are driven by a trade-off between labor supply distortions at the intensive margin and at the extensive margin, which has not been discussed in the previous literature.

Importantly, the paper has shown that an EITC can be optimal although society considers the unemployed strictly more deserving than the working poor, and the working poor more deserving than high-income earners. It has repeatedly been argued that society might in contrast consider the working poor more deserving than the unemployed (for example, see the arguments in Beaudry et al. 2009 and Saez \& Stantcheva 2016). As a result of the trade-off between distortions at both margins, redistributive concerns of this kind would not only make a stronger case for negative participation taxes, but also for negative marginal taxes. Hence, the results of this paper would even be reinforced.

This paper is the first to analytically confirm Saez (2002)'s conjecture that an EITC can be optimal when the standard model is extended to take into account labor supply responses at both margins. Still, the paper abstracts from some aspects that could affect the desirability of in-work benefit schemes such as the EITC. For example, an EITC might have additional benefits when there is learning on the job, i.e., when larger output provision today increases an agent's labor productivity tomorrow (see Best \& Kleven 2013). An EITC might also be more desirable if an agent's non-participation in the labor market has negative external effects, e.g., on the educational outcomes of his children (see Ho \& Pavoni 2016, Nichols \& Rothstein 2015. 37-40). Future research should incorporate these non-standard aspects into empirically plausible two-margin models in order to investigate their interaction with the mechanism studied in this paper. 


\section{References}

Beaudry, P., Blackorby, C. \& Szalay, D. (2009), 'Taxes and employment subsidies in optimal redistribution programs', American Economic Review 99(1), 216-42.

Best, M. \& Kleven, H. (2013), Optimal income taxation with career effects of work effort, SSRN Working Paper. URL: http://papers.ssrn.com/sol3/papers.cfm? abstract_id=2051214.

Blau, F. D. \& Kahn, L. M. (2007), 'Changes in the labor supply behavior of married women: 1980-2000', Journal of Labor Economics 25, 393-438.

Brett, C. \& Weymark, J. (2017), 'Voting over selfishly optimal nonlinear income tax schedules', Games and Economic Behavior 101, 172-188. Special Issue in Honor of John O. Ledyard.

Brewer, M., Saez, E. \& Shephard, A. (2010), Means testing and tax rates on earnings, in J. Mirrlees, S. Adam, T. Besley, R. Blundell, S. Bond, R. Chote, M. Gammie, P. Johnson, G. Myles \& J. Poterba, eds, 'Dimensions of Tax Design: The Mirrlees Review', Oxford University Press, chapter 2, pp. 90-201.

Choné, P. \& Laroque, G. (2005), 'Optimal incentives for labor force participation', Journal of Public Economics 89(2-3), 395-425.

Choné, P. \& Laroque, G. (2010), 'Negative marginal tax rates and heterogeneity', American Economic Review 100(5), 2532-47.

Choné, P. \& Laroque, G. (2011), 'Optimal taxation in the extensive model', Journal of Economic Theory 146(2), 425-453.

Christiansen, V. (2015), 'Optimal participation taxes', Economica 82, 595-612.

Diamond, P. (1980), 'Income taxation with fixed hours of work', Journal of Public Economics 13(1), 101-110.

Eissa, N. \& Hoynes, H. W. (2004), 'Taxes and the labor market participation of married couples: the earned income tax credit', Journal of Public Economics 88, 1931-1958.

Eissa, N. \& Hoynes, H. W. (2006), Behavioral responses to taxes: Lessons from the eitc and labor supply, in J. M. Poterba, ed., 'Tax Policy and the Economy', Vol. 20, MIT Press, chapter 3, pp. 73-110. 
Executive Office of the President and U.S. Treasury Department (2014), The President's Proposal to Expand the Earned Income Tax Credit, March 3, 2014. URL: https://obamawhitehouse.archives.gov/sites/default/files/docs/ eitc_report_o.pdf.

Hellwig, M. F. (2007), 'A contribution to the theory of optimal utilitarian income taxation', Journal of Public Economics 91(7-8), 1449-1477.

Ho, C. \& Pavoni, N. (2016), Efficient child care subsidies, IGIER Working Paper No. 572, Bocconi University.

Hotz, V. J. \& Scholz, J. K. (2003), The earned income tax credit, in R. A. Moffitt, ed., 'Means-Tested Transfer Programs in the United States', University of Chicago Press, chapter 3, pp. 141-198.

House Budget Committee Majority Staff, Chairman Paul Ryan (2014), Expanding Opportunity in America. A Discussion Draft from the House Budget Committee, July 24, 2014. URL: http://budget.house.gov/opportunity/.

Immervoll, H., Kleven, H. J., Kreiner, C. T. \& Saez, E. (2007), 'Welfare reform in european countries: A microsimulation analysis', The Economic Journal $\mathbf{1 1 7}(516), 1-44$.

Jacquet, L., Lehmann, E. \& Van der Linden, B. (2013), 'Optimal redistributive taxation with both extensive and intensive responses', Journal of Economic Theory 148(5), 1770-1805.

Juhn, C., Murphy, K. M. \& Topel, R. H. (1991), 'Why has the natural rate of unemployment increased over time?', Brookings Papers on Economic Activity 1991(2), pp. 75-142.

Juhn, C., Murphy, K. M. \& Topel, R. H. (2002), 'Current unemployment, historically contemplated', Brookings Papers on Economic Activity 2002(1), pp. 79-136.

Laroque, G. (2005), 'Income maintenance and labor force participation', Econometrica $73(2), 341-376$.

Lehmann, E., Simula, L. \& Trannoy, A. (2014), 'Tax me if you can! optimal nonlinear income tax between competing governments', Quarterly Journal of Economics 129(4), 1995-2030. 
Lorenz, N. \& Sachs, D. (2012), Optimal participation taxes and efficient transfer phase-out, Working Paper Series of the Department of Economics, University of Konstanz 2012-37. URL: http://ideas.repec.org/p/knz/dpteco/1237.html.

McClelland, R. \& Mok, S. (2012), A review of recent research on labor supply elasticities, Congressional Budget Office Working Paper 2012-12, Congressional Budget Office. URL: https://www.cbo.gov/publication/43675.

Meghir, C. \& Phillips, D. (2010), Labour supply and taxes, in J. Mirrlees, S. Adam, T. Besley, R. Blundell, S. Bond, R. Chote, M. Gammie, P. Johnson, G. Myles \& J. Poterba, eds, 'Dimensions of Tax Design: The Mirrlees Review', Oxford University Press, chapter 3, pp. 202-274.

Meyer, B. D. \& Rosenbaum, D. T. (2001), 'Welfare, the Earned Income Tax Credit and the labor supply of single mothers', Quarterly Journal of Economics 116(3), 1063-1114.

Mirrlees, J. A. (1971), 'An exploration in the theory of optimum income taxation', Review of Economic Studies 38(114), 175-208.

Nichols, A. \& Rothstein, J. (2015), The Earned Income Tax Credit (EITC), NBER Working Paper Series No. 21211. URL: http://www.nber.org/papers/w21211.

Piketty, T. \& Saez, E. (2013), Optimal labor income taxation, in A. J. Auerbach, R. Chetty, M. Feldstein \& E. Saez, eds, 'Handbook of Public Economics', Vol. 5, Elsevier, chapter 7, pp. 391-474.

Rochet, J.-C. \& Stole, L. A. (2002), 'Nonlinear pricing with random participation', Review of Economic Studies 69(1), 277-311.

Saez, E. (2002), 'Optimal income transfer programs: Intensive versus extensive labor supply responses', The Quarterly Journal of Economics 117(3), 1039-1073.

Saez, E. \& Stantcheva, S. (2016), 'Generalized social welfare weights for optimal tax theory', American Economic Review 106(1), 24-45.

Seade, J. (1982), 'On the sign of the optimum marginal income tax', Review of Economic Studies 49(4), 637-43.

Seade, J. K. (1977), 'On the shape of optimal tax schedules', Journal of Public Economics 7(2), 203-235.

Stiglitz, J. E. (1982), 'Self-selection and pareto efficient taxation', Journal of Public Economics 17(2), 213-240. 


\section{Appendix}

\section{A Proofs}

\section{Proof of Lemma 1}

Proof. Using equation (1), incentive compatibility requires that, for all pairs of $(\omega, \delta)$ and $\left(\omega^{\prime}, \delta^{\prime}\right)$ in $\Omega \times \Delta$,

$$
c(\omega, \delta)-h[y(\omega, \delta), \omega]-1_{y(\omega, \delta)>0} \delta \geq c\left(\omega^{\prime}, \delta^{\prime}\right)-h\left[y\left(\omega^{\prime}, \delta^{\prime}\right), \omega\right]-1_{y\left(\omega^{\prime}, \delta^{\prime}\right)>0} \delta .
$$

To satisfy these constraints, first, all pairs of agents with types $\left(\omega_{j}, \delta\right)$ and $\left(\omega_{j}, \delta^{\prime}\right)$ who provide strictly positive output must receive the same gross (of fixed costs) utility $c\left(\omega_{j}, \delta\right)-$ $h\left[y\left(\omega_{j}, \delta\right), \omega_{j}\right]=z_{j}$. Second, all agents with types $(\omega, \delta)$ and $\left(\omega^{\prime}, \delta^{\prime}\right)$ who provide zero output must receive the same consumption $c(\omega, \delta)=c\left(\omega^{\prime}, \delta^{\prime}\right)=c_{0}$. Third, an allocation can only be incentive-compatible if each agent with skill type $\omega_{j}$ and fixed cost type below (above) $\delta_{j}=z_{j}-c_{0}$ provides positive output (zero output).

Second-best Pareto efficiency requires, moreover, that all agents with skill type $\omega_{j}$ and fixed cost type below $\delta_{j}$ receive the same bundle $\left(c_{j}, y_{j}\right)$. By the strict convexity of effort cost function $h$, there is a unique bundle $\left(c_{j}, y_{j}\right)$ that minimizes the net transfer $c-y$, subject to $c-h\left(y, \omega_{j}\right)=z_{j}$ and to the IC constraints along the skill dimension, $z_{k} \geq c-h\left(y, \omega_{k}\right)$, for all $k \neq j$. Assume there is an initial allocation in which some agent with type $\left(\omega_{j}, \delta\right)$ receives bundle $\left(c^{\prime}, y^{\prime}\right) \neq\left(c_{j}, y_{j}\right)$ with $y^{\prime}>0$. Changing his allocation to $\left(c_{j}, y_{j}\right)$ allows to save resources without changing his utility level, and to redistribute these resources lump-sum to all agents in the economy. Hence, the initial allocation with $\left(c^{\prime}, y^{\prime}\right) \neq\left(c_{j}, y_{j}\right)$ was not Pareto efficient.

\section{Proof of Lemma 2}

Proof. In every implementable allocation, the downward IC constraint between the workers with skill types $\omega_{j}$ and $\omega_{j+1}$ is satisfied, i.e.,

$$
\begin{array}{rlrl} 
& & c_{j+1}-h\left(y_{j+1}, \omega_{j+1}\right) & \geq c_{j}-h\left(y_{j}, \omega_{j+1}\right) \\
\Leftrightarrow & \delta_{j+1}-\delta_{j} & \geq h\left(y_{j}, \omega_{j}\right)-h\left(y_{j}, \omega_{j+1}\right) .
\end{array}
$$

By $h_{\omega}(y, \omega)<0$, this implies that $\delta_{j+1}>\delta_{j}$. Parts (i) and (ii) of Condition 1 ensure that $\eta_{j}(c, y)=\frac{g_{j}\left(\delta_{j}\right)}{G_{j}\left(\delta_{j}\right)}>\frac{g_{j}\left(\delta_{j+1}\right)}{G_{j}\left(\delta_{j+1}\right)} \geq \frac{g_{j+1}\left(\delta_{j+1}\right)}{G_{j+1}\left(\delta_{j+1}\right)}=\eta_{j+1}(c, y)$.

\section{Proof of Lemma 3}

The proof of Lemma 3 consists of three steps. I start with two preliminary Lemmas. 
Lemma 7. Consider the relaxed problem formally defined in section 5.1. For any $\alpha \in \mathcal{A}$ such that an interior solution $\left(c^{\alpha R}, y^{\alpha R}\right)$ exists, it satisfies for each $j \in J$ the conditions

$$
\begin{aligned}
h_{y}\left(y_{j}^{\alpha R}, \omega_{j}\right) & =1, \\
\delta_{j}^{\alpha R}-\delta^{*}\left(\omega_{j}\right) & =\frac{\alpha_{j}-1}{A_{j}\left(\delta_{j}^{\alpha R}\right)}, \text { and } \\
c_{0}^{\alpha R} & =\sum_{j=1}^{2} f_{j} G_{j}\left(\delta_{j}^{\alpha R}\right)\left(\delta^{*}\left(\omega_{j}\right)-\delta_{j}^{\alpha R}\right) .
\end{aligned}
$$

Proof. Assume that the relaxed problem has a solution for social weights $\alpha$. First, $\left(c^{\alpha R}, y^{\alpha R}\right)$ must be Pareto-efficient in the set of allocations that are feasible and satisfy the IC constraints (14) and (15). Second, the statements in 1 must be true for $\left(c^{\alpha R}, y^{\alpha R}\right)$ because its proof only uses the IC constraints (14) and (15). Consequently, the Lagrangian of the relaxed problem can be written as

$$
\begin{aligned}
\mathcal{L}= & \sum_{j=1}^{n} f_{j}\left(\int_{\underline{\delta}}^{\delta_{j}} g_{j}(\delta) \gamma(\omega, \delta) \Psi\left[c_{j}-h\left(y_{j}, \omega_{j}\right)-\delta\right] d \delta+\int_{\delta_{j}}^{\bar{\delta}} g_{j}(\delta) \gamma(\omega, \delta) \Psi\left(c_{0}\right) d \delta\right) \\
& +\lambda\left[\sum_{j=1}^{n} f_{j} G_{j}\left(\delta_{j}\right)\left(y_{j}-c_{j}+c_{0}\right)-c_{0}\right],
\end{aligned}
$$

where $\delta_{j}=c_{j}-h\left(y_{j}, \omega_{j}\right)-c_{0}$ and $\lambda$ is the Lagrange parameter associated with the feasibility condition. Replacing the average weights $\bar{\alpha}_{j}$ and $\bar{\alpha}_{0}$ as defined in 10) and (11) by the exogenous numbers $\alpha_{j}$ and $\alpha_{0}$, the first-order conditions of this problem are given by

$$
\begin{array}{ll}
\mathcal{L}_{c_{j}}=f_{j}\left[G_{j}\left(\delta_{j}^{\alpha R}\right)\left(\alpha_{j}-\lambda\right)+\lambda g_{j}\left(\delta_{j}^{\alpha R}\right)\left(y_{j}^{\alpha R}-c_{j}^{\alpha R}+c_{0}^{\alpha R}\right)\right] & \stackrel{!}{=} 0 \\
\mathcal{L}_{y_{j}}=f_{j}\left[-h_{y}\left(y_{j}^{\alpha R}, \omega_{j}\right)\left(G_{j}\left(\delta_{j}^{\alpha R}\right) \alpha_{j}+\lambda g_{j}\left(\delta_{j}^{\alpha R}\right)\left(y_{j}^{\alpha R}-c_{j}^{\alpha R}+c_{0}^{\alpha R}\right)\right)+\lambda G_{j}\left(\delta_{j}^{\alpha R}\right)\right] & \stackrel{!}{=} 0 \\
\mathcal{L}_{c_{0}}=\sum_{j=1}^{n} f_{j}\left[\left(1-G_{j}\left(\delta_{j}^{\alpha R}\right)\right) \alpha_{0}+\lambda G_{j}\left(\delta_{j}^{\alpha R}\right)-\lambda g_{j}\left(\delta_{j}^{\alpha R}\right)\left(y_{j}^{\alpha R}-c_{j}^{\alpha R}+c_{0}^{\alpha R}\right)-1\right] & \stackrel{!}{=} 0
\end{array}
$$

Equation 27) follows from combining the first-order conditions with respect to $c_{j}$ and $y_{j}$. Combining the FOCs with respect to $c_{j}$ for all $j \in J$ and $c_{0}$ gives

$$
\lambda=\sum_{j=1}^{n} f_{j}\left[G_{j}\left(\delta_{j}^{\alpha R}\right) \alpha_{j}+\left(1-G_{j}\left(\delta_{j}^{\alpha R}\right)\right) \alpha_{0}\right]=\alpha_{M}=1,
$$

where the average weight $\alpha_{M}$ is normalized to 1 for all $\alpha \in \mathcal{A}$. Using $\lambda=1$ and $c_{j}=$ $\delta_{j}+h\left(y_{j}, \omega_{j}\right)+c_{0}$, the FOC with respect to $c_{j}$ can be rewritten as

$$
\delta_{j}^{\alpha R}-y_{j}^{\alpha R}+h\left(y_{j}^{\alpha R}, \omega_{j}\right)=\frac{G_{j}\left(\delta_{j}^{\alpha R}\right)}{g_{j}\left(\delta_{j}^{\alpha R}\right)}\left(\alpha_{j}-1\right) .
$$


As $A_{j}(\delta)=\frac{g_{j}(\delta)}{G_{j}(\delta)}$ and $\delta^{*}\left(\omega_{j}\right)=\max _{y>0}\left\{y-h\left(y, \omega_{j}\right)\right\}=y_{j}^{\alpha R}-h\left(y_{j}^{\alpha R}, \omega_{j}\right)$, this is identical to equation (28). Finally, equation 29) follows from substituting $-\delta_{j}^{\alpha R}-h\left(y_{j}^{\alpha R}, \omega_{j}\right)=$ $-c_{j}^{\alpha R}+c_{0}^{\alpha R}$ into the feasibility condition.

Lemma 8. There is a number $\chi \in(1,2]$ such that the relaxed optimal tax problem has a unique interior solution for $\alpha$ if $\alpha_{j}<\chi$ for all $j \in J$.

Proof. As shown above, the FOC with respect to $c_{j}$ is identical to

$$
k_{j}\left(\delta_{j}^{\alpha R}, \alpha_{j}\right)=G_{j}\left(\delta_{j}^{\alpha R}\right)\left(\alpha_{j}-1\right)+g_{j}\left(\delta_{j}^{\alpha R}\right)\left(\delta^{*}\left(\omega_{j}\right)-\delta_{j}^{\alpha R}\right)=0
$$

for every $j \in J$. Hence, the relaxed problem has a unique interior extremum for $\alpha$ if and only if $k_{j}\left(\delta, \alpha_{j}\right)$ has a unique root in $\delta$ with $\delta \in(\underline{\delta}, \bar{\delta})$. First, $k_{j}$ is continuous in $\alpha_{j}$ and $\delta$ for all $\delta \in(\underline{\delta}, \bar{\delta})$. Second, $k_{j}\left(\underline{\delta}, \alpha_{j}\right)>0$ for any $\alpha_{j} \geq 0$ because $\delta^{*}\left(\omega_{j}\right) \geq \delta^{*}\left(\omega_{1}\right)>\underline{\delta}$ for all $j \in J$ by (2) and the properties of $h$. Third, the derivative of $k_{j}$ with respect to $c_{j}$ (or its first argument) is given by

$$
k_{j \delta}\left(\delta, \alpha_{j}\right)=g_{j}(\delta)\left(\alpha_{j}-2\right)+g_{j}^{\prime}(\delta)\left(\delta^{*}\left(\omega_{j}\right)-\delta\right) .
$$

If $k$ has a root at $\delta^{\prime}$, this derivative has to be equal to

$$
k_{j \delta}\left(\delta^{\prime}, \alpha_{j}\right)=G_{j}\left(\delta^{\prime}\right)\left[A_{j}\left(\delta^{\prime}\right)\left(\alpha_{j}-2\right)-a_{j}\left(\delta^{\prime}\right)\left(\alpha_{j}-1\right)\right],
$$

where $A_{j}(\delta)=\frac{g_{j}(\delta)}{G_{j}(\delta)}$ and $a_{j}(\delta)=\frac{g_{j}^{\prime}(\delta)}{g_{j}(\delta)}$. Part (1) of Condition 1 ensures that $A_{j}(\delta)>a_{j}(\delta)$ for all $\delta \in \Delta$ and all $j \in J$. This implies that $k_{j \delta}\left(\alpha_{j}, \delta^{\prime}\right)$ is negative if

$$
\alpha_{j}<\underline{\chi}_{j}\left(\delta^{\prime}\right):=1+\frac{A_{j}\left(\delta^{\prime}\right)}{A_{j}\left(\delta^{\prime}\right)-a_{j}\left(\delta^{\prime}\right)}
$$

and that $\underline{\chi}_{j}\left(\delta^{\prime}\right)>1$ for all $\delta^{\prime} \in \Delta$ and all $j \in J$. Define $\chi_{j}$ as the minimum of $\underline{\chi}_{j}\left(\delta^{\prime}\right)$ over $\delta^{\prime} \in(\underline{\delta}, \bar{\delta}]$. By assumption, $g_{j}(\delta)$ is larger than some number $g>0$ for all $\delta \in \Delta$ and $g_{j}^{\prime}(\delta) \leq 0$ for some $\delta \in \Delta$. By the first property, $\chi_{j}$ is bounded away from 1 for all $j \in J$. By the second property, $\chi_{j} \leq 2$ for all $j \in J$. Consequently, $k_{j}\left(\delta, \alpha_{j}\right)$ has at most one root in $\delta$ if $\alpha_{j}<\chi_{j}$. If this root exists, it constitutes a maximum because $k_{j \delta}\left(\delta, \alpha_{j}\right)<0$.

The existence of a root is ensured if, additionally, $k_{j}\left(\bar{\delta}, \alpha_{j}\right)<0$, which is ensured for $\alpha_{j}<\chi_{j}^{\prime}:=1+g_{j}(\bar{\delta})\left(\bar{\delta}-\delta^{*}\left(\omega_{j}\right)\right)$. Note that $\chi_{j}^{\prime}>1$ for all $j \in J$ by (3). Let $\chi$ be given by the minimum of $\chi_{j}$ and $\chi_{j}^{\prime}$ over all $j \in J$. By the construction of $\chi$, the relaxed problem has a unique interior extremum for $\alpha$ if $\alpha_{j}<\chi$ for all $j \in J$.

Lemma 3 follows as a corollary from the Lemmas 7 and 8 , using the definitions of labor supply distortions at both margins provided in Section 3.2. In particular, equation 27) implies that labor supply is undistorted at the intensive margin in all skill groups. Equation (28) implies that labor supply is downwards (upwards) distorted at the extensive margin in skill group $j \in J$ if and only if $\alpha_{j}$ is below (above) 1 . 


\section{Proof of Lemma 4}

For the following analysis, it proves useful to rewrite the downward IC constraint between the workers in skill groups $j$ and $j+1$ as (26). Inserting equations (27) and (28) into (26), the downward IC constraint is violated if

$$
\begin{aligned}
\delta^{*}\left(\omega_{j+1}\right)+\frac{\alpha_{j+1}-1}{A_{j+1}\left(\delta_{j+1}^{\alpha R}\right)}-\delta^{*}\left(\omega_{j}\right)-\frac{\alpha_{j}-1}{A_{j}\left(\delta_{j}^{\alpha R}\right)}<h\left(y_{j}^{\alpha R}, \omega_{j}\right)-h\left(y_{j}^{\alpha R}, \omega_{j+1}\right) \\
\Leftrightarrow \frac{\alpha_{j+1}-1}{A_{j+1}\left(\delta_{j+1}^{\alpha R}\right)}-\frac{\alpha_{j}-1}{A_{j}\left(\delta_{j}^{\alpha R}\right)}<\left[y_{j}^{\alpha R}-h\left(y_{j}^{\alpha R}, \omega_{j+1}\right)\right]-\delta^{*}\left(\omega_{j+1}\right) .
\end{aligned}
$$

Replacing $\eta_{j}\left(c^{\alpha R}, y^{\alpha R}\right)=A_{j}\left(\delta_{j}^{\alpha R}\right)$ and $\delta^{*}\left(\omega_{j+1}\right)=y_{j+1}^{\alpha R}-h\left(y_{j+1}^{\alpha R}, \omega_{j+1}\right)$, the last line is identical to (18).

Correspondingly, the upward IC constraint can be rewritten as

$$
\delta_{j+1}-\delta_{j} \leq h\left(y_{j+1}, \omega_{j}\right)-h\left(y_{j+1}, \omega_{j+1}\right)
$$

Inserting (27) and (28) into this constraint and replacing $\delta^{*}\left(\omega_{j}\right)$ gives equation (19).

\section{Proof of Lemma 5}

Lemma 5 is proven through Lemmas 9 to 14.

Lemma 9. For every $j \in J$ and every $\alpha \in \mathcal{A}^{\chi}, \delta_{j}^{\alpha R}$ is strictly increasing in $\alpha_{j}$.

Proof. Threshold $\delta_{j}^{\alpha R}$ is implicitly defined by condition 28 in Lemma 7. Using the implicit function theorem, its derivative with respect to $\alpha_{j}$ is given by

$$
\frac{d \delta_{j}^{\alpha R}}{d \alpha_{j}}=\frac{A_{j}\left(\delta_{j}^{\alpha R}\right)^{-1}}{1+\left(1-\alpha_{j}\right)\left(1-\frac{a_{j}\left(\delta_{j}^{\alpha R}\right)}{A_{j}\left(\delta_{j}^{\alpha R}\right)}\right)}=\frac{1}{\left(2-\alpha_{j}\right) A_{j}\left(\delta_{j}^{\alpha R}\right)-\left(1-\alpha_{j}\right) a_{j}\left(\delta_{j}^{\alpha R}\right)}
$$

where the numerator equals $-k_{\delta}\left(\alpha_{j}, \delta_{j}^{\alpha R}\right) / G_{j}\left(\hat{\delta}_{j}^{\alpha R}\right)>0$ (see proof of Lemma 8). Hence, the derivative is strictly positive for all $\alpha_{j}<\chi$.

Lemma 10. For any skill group $j \in J_{-n}$, if $\alpha_{j}=\alpha_{j+1}=\alpha^{\prime}$,

(i) the difference $\delta_{j+1}^{\alpha R}-\delta_{j}^{\alpha R}$ is strictly increasing in $\alpha^{\prime}$ for all $\alpha^{\prime} \in(0, \chi)$ such that the downward IC constraint (26) between skill groups $j$ and $j+1$ is satisfied;

(ii) $\left(c^{\alpha R}, y^{\alpha R}\right)$ satisfies the downward IC constraint (26) between skill groups $j$ and $j+1$ for all $\alpha^{\prime} \in[1, \chi)$;

(iii) $\left(c^{\alpha R}, y^{\alpha R}\right)$ satisfies the upward IC constraint (32) between skill groups $j$ and $j+1$ for all $\alpha^{\prime} \in[0,1]$. 
Proof. (i) Using (33), the derivative of $\delta_{j+1}^{\alpha R}\left(\alpha^{\prime}\right)-\delta_{j}^{\alpha R}\left(\alpha^{\prime}\right)$ with respect to $\alpha^{\prime}$ is strictly positive if

$$
\left(2-\alpha^{\prime}\right)\left[A_{j}\left(\delta_{j}^{\alpha R}\right)-A_{j+1}\left(\delta_{j+1}^{\alpha R}\right)\right]>\left(1-\alpha^{\prime}\right)\left[a_{j}\left(\delta_{j}^{\alpha R}\right)-a_{j+1}\left(\delta_{j+1}^{\alpha R}\right)\right] .
$$

In all allocations that satisfy the downward IC constraint, $\delta_{j+1}^{\alpha R}>\delta_{j}^{\alpha R}$. By Conditions 1 and 2 , we hence have $A_{j}\left(\delta_{j}^{\alpha R}\right)-A_{j+1}\left(\delta_{j+1}^{\alpha R}\right)>0$ and $a_{j}\left(\delta_{j}^{\alpha R}\right)-a_{j+1}\left(\delta_{j+1}^{\alpha R}\right) \geq 0$. Because $\chi \in(1,2]$, this directly implies that inequality (34) is satisfied for any $\alpha^{\prime} \in[1, \chi)$. Moreover, Condition 2 ensures that $a_{j}\left(\delta_{j}^{\alpha R}\right)-a_{j+1}\left(\delta_{j+1}^{\alpha R}\right) \leq 2\left[A_{j}\left(\delta_{j}^{\alpha R}\right)-\right.$ $\left.A_{j+1}\left(\delta_{j+1}^{\alpha R}\right)\right]$. Hence, inequality (34) is also satisfied for the alternative case $\alpha^{\prime} \in$ $(0,1)$, where $\left(2-\alpha^{\prime}\right) /\left(1-\alpha^{\prime}\right)>2$.

(ii) For $\alpha_{j}=1, \delta_{k}^{\alpha R}=\delta^{*}\left(\omega_{k}\right)$ for $k \in\{j, j+1\}$. Inserting these into the downward IC constrait (26) and rearranging terms gives

$$
\delta^{*}\left(\omega_{j+1}\right)=y_{j+1}^{\alpha R}-h\left(y_{j+1}^{\alpha R}, \omega_{j+1}\right) \geq y_{j}^{\alpha R}-h\left(y_{j}^{\alpha R}, \omega_{j+1}\right),
$$

which holds with strict inequality by the single-crossing property. The left-hand side of (26) is given by $\delta_{j+1}^{\alpha R}-\delta_{j}^{\alpha R}$, which is strictly increasing in $\alpha^{\prime}$ by part (i), while the right-hand side is constant. Hence, the downward IC constraint is satisfied for all $\alpha^{\prime} \geq 1$.

(iii) For $\alpha_{j}=1$, inserting $\delta_{k}^{\alpha R}=\delta^{*}\left(\omega_{k}\right)$ for $k \in\{j, j+1\}$ into the upward IC constraint (32) gives $y_{j+1}^{\alpha R}-h\left(y_{j+1}^{\alpha R}, \omega_{j}\right) \leq \delta^{*}\left(\omega_{j}\right)=\max _{y>0}\left\{y-h\left(y, \omega_{j}\right)\right\}$, which is again satisfied with strict inequality. As the left-hand side of $(32)$ is strictly increasing in $\alpha^{\prime}$, the upward IC is satisfied for all $\alpha^{\prime}<1$ such that the downward IC is satisfied. As the downward and upward IC constraints cannot be violated at the same time, the upward IC constrained is also satisfied for all other $\alpha^{\prime}<1$.

Lemma 11. Consider the downward IC constraint (26) between the workers with skill types $\omega_{j}$ and $\omega_{j+1}=a_{j} \omega_{j}$ for some $j \in J_{-n}$. Define $a_{j}^{0}$ as the supremum of the set of real numbers $a^{\prime}>1$ such that, given $\alpha_{j}=\alpha_{j+1}=0,\left(c^{\alpha R}, y^{\alpha R}\right)$ violates the downward $I C$ constraint for all $a_{j} \in\left(1, a^{\prime}\right)$.

(i) If Condition 1 is satisfied, $a_{j}^{0}>1$ exists.

(ii) Let $\alpha_{j}=\alpha_{j+1}=\alpha^{\prime}$. For each $a_{j} \in\left(1, a_{j}^{0}\right)$, there exists a unique number $\underline{\beta}_{j} \in(0,1)$ such that $\left(c_{\alpha}, y_{\alpha}\right)$ violates the downward IC constraint (16) if and only if $\alpha^{\prime}<\underline{\beta}_{j}$.

Proof. (i) In the limit case $a_{j}=1$, the downward IC constraint 26) simplifies to $\delta_{j+1}^{\alpha R}-$ $\delta_{j}^{\alpha R} \geq 0$. By 28$) \delta_{k}^{\alpha R}$ is implicitly defined by $\frac{1}{A_{k}\left(\delta_{k}^{\alpha R}\right)}+\delta_{k}^{\alpha R}=\delta^{*}\left(\omega_{k}\right)$ for $\alpha^{\prime}=0$ and any $k \in J$. For $a_{j}=1$, we have $\delta^{*}\left(\omega_{j+1}\right)=\delta^{*}\left(\omega_{j}\right)$. By Condition 11 (ii), 
$A_{j}(\delta) \geq A_{j+1}(\delta)$. Hence, there are two possible cases. First, if $A_{j}\left(\delta_{j}^{\alpha R}\right)>A_{j+1}\left(\delta_{j}^{\alpha R}\right)$, we have $\delta_{j+1}^{\alpha R}<\delta_{j}^{\alpha R}$. Hence, the downward IC constraint is violated for $\alpha^{\prime}=0$ and $a=1$. Second, if $A_{j}\left(\delta_{j}^{\alpha R}\right)=A_{j+1}\left(\delta_{j}^{\alpha R}\right)$, we have $\delta_{j+1}^{\alpha R}=\delta_{j}^{\alpha R}$. Hence, the downward IC constraint is satisfied with equality for $\alpha^{\prime}=0$ and $a=1$.

To consider the general case $a_{j}>1$, I compare the derivatives of both sides of 26 with respect to $a_{j}$ at $a_{j}=1$. The derivative of the left-hand side is given by

$$
\frac{\frac{d \delta^{*}\left(\omega_{j+1}\right)}{d a_{j}}}{2-\frac{a_{j+1}\left(\delta_{j+1}^{\alpha R}\right)}{A_{j+1}\left(\delta_{j+1}^{\alpha R}\right)}}=\frac{\frac{d\left[y_{j+1}^{\alpha R}-h\left(y_{j+1}^{\alpha R}, \omega_{j} a_{j}\right)\right]}{d a_{j}}}{2-\frac{a_{j+1}\left(\delta_{j+1}^{\alpha R}\right)}{A_{j+1}\left(\delta_{j+1}^{\alpha R}\right)}}=-\frac{\omega_{j} h_{\omega}\left(y_{j+1}^{\alpha R}, \omega_{j} a\right)}{2-\frac{a_{j+1}\left(\delta_{j+1}^{\alpha R}\right)}{A_{j+1}\left(\delta_{j+1}^{\alpha R}\right)}}>0
$$

where the term in the denominator is strictly larger than 1 because $a_{j+1}(\delta)<A_{j+1}(\delta)$ for all $\delta \in \Delta$ by Condition 1 (ii). The derivative of the right-hand side is given by $-\omega_{j} h_{\omega}\left(y_{j}^{\alpha R}, \omega_{j} a\right)>0$, which is strictly larger than the derivative of the left-hand side. Hence, the downward IC constraint is unambiguously violated for $\alpha_{j}=\alpha_{j+1}=$ 0 and all $a_{j}$ between 1 and some number $a^{\prime}>1$. The supremum $a_{j}^{0}$ is hence welldefined, and may either be given by a finite number or by $\infty$.

(ii) For any $a_{j} \in\left(1, a_{j}^{0}\right)$, the right-hand side of 26) is independent of $\alpha^{\prime}$. The left-hand side $\delta_{j+1}^{\alpha R}-\delta_{j}^{\alpha R}$ is small enough to violate the downward IC constraint for $\alpha^{\prime}=0$ by part (i), and large enough to satisfy this constraint with strict inequality for $\alpha^{\prime}=1$ by Lemma 10 (ii). By Lemma 10 (i), $\delta_{j+1}^{\alpha R}-\delta_{j}^{\alpha R}$ is strictly increasing in $\alpha^{\prime}$ for all levels of $\alpha^{\prime}$ such that (26) is satisfied. Consequently, there exists a unique threshold $\underline{\beta}_{j} \in(0,1)$ such that the downward IC constraint is violated for all $\alpha^{\prime} \in\left[0, \underline{\beta}_{j}\right)$ and satisfied for all $\alpha^{\prime} \in\left[\underline{\beta}_{j}, \chi\right)$.

Lemma 12. Consider the upward IC constraint (17) between the workers with skill types $\omega_{j}$ and $\omega_{j+1}=a_{j} \omega_{j}$ for some $j \in J_{-n}$. Define $a_{j}^{u}(\gamma)$ as the supremum of the set of real numbers $a^{\prime}>1$ such that, given $\alpha_{j}=\alpha_{j+1}=\gamma>1,\left(c^{\alpha R}, y^{\alpha R}\right)$ violates the upward IC constraint for all $a_{j} \in\left(1, a^{\prime}\right)$.

(i) If Condition 1 is satisfied, $a_{j}^{u}(\gamma)$ exists for all $\gamma \in(1, \chi)$.

(ii) Let $\alpha_{j}=\alpha_{j+1}=\alpha^{\prime}$. For each $\gamma \in(1, \chi)$ and each $a \in\left(1, a_{j}^{u}(\gamma)\right)$, there exists a unique number $\bar{\beta}_{j} \in(1, \gamma)$ such that $\left(c_{\alpha}, y_{\alpha}\right)$ violates the upward $I C$ constraint if and only if $\alpha^{\prime} \in\left(\bar{\beta}_{j}, \chi\right)$.

Proof. (i) Fix a number $\gamma \in(1, \chi)$. In the limit case $a_{j}=1$, the upward IC constraint (32) simplifies to $\delta_{j+1}^{\alpha R}-\delta_{j}^{\alpha R} \leq 0$. For $\alpha_{j}=\alpha_{j+1}=\gamma>1, \delta_{k}^{\alpha R}$ is implicitly defined by $\frac{1-\gamma}{A_{k}\left(\delta_{k}^{\alpha R}\right)}+\delta_{k}^{\alpha R}=\delta^{*}\left(\omega_{k}\right)$ for $k \in\{j, j+1\}$. For $a=1, \delta^{*}\left(\omega_{j}\right)=\delta^{*}\left(\omega_{j+1}\right)$. By Condition 1 (ii), $A_{j}(\delta) \geq A_{j+1}(\delta)$ for all $\delta \in \Delta$. Again, there are two possible cases. First, if $A_{j}\left(\delta_{j}^{\alpha R}\right)>A_{j+1}\left(\delta_{j}^{\alpha R}\right)$, we have $\delta_{j+1}^{\alpha R}>\delta_{j}^{\alpha R}$ so that (32) is violated. Second, 
if $A_{j}\left(\delta_{j}^{\alpha R}\right)=A_{j+1}\left(\delta_{j}^{\alpha R}\right)$, we have $\delta_{j+1}^{\alpha R}=\delta_{j}^{\alpha R}$ so that 32 is satisfied with strict equality.

To consider the general case $a_{j}>1$, I again compare the derivatives of both sides of (32) in $a_{j}$, given a fixed $\gamma$. The derivative of the left-hand side is given by

$$
\frac{-\omega_{j} h_{\omega}\left(y_{j+1}^{\alpha R}, a_{j} \omega_{j}\right)}{1-(\gamma-1)\left(1-\frac{a_{j+1}\left(\delta_{j+1}^{\alpha R}\right)}{A_{j+1}\left(\delta_{j+1}^{\alpha R}\right)}\right)}>0,
$$

where the term in the denominator is strictly positive for any $\gamma<\chi$ and strictly smaller than 1 by Condition 1 (ii) for all $\gamma>1$. The derivative of the right-hand side is given by

$$
-\omega_{j} h_{\omega}\left(y_{j+1}^{\alpha R}, a_{j} \omega_{j}\right)+\left[h\left(y_{j+1}^{\alpha R}, \omega_{j}\right)-h\left(y_{j+1}^{\alpha R}, a_{j} \omega_{j}\right)\right] \frac{d y_{j+1}^{\alpha R}}{d a_{j}}>0 .
$$

At $a_{j}=1$, the term in squared brackets equals zero. Hence, the derivative of the left-hand side is strictly larger than the derivative of the right-hand side of 32 . Thus, the upward CI constraint is violated for $\alpha_{j}=\alpha_{j+1}=\gamma>1$ and all $a_{j}$ between 1 and some number $a^{\prime}>1$. The supremum $a_{j}^{u}(\gamma)$ is hence well-defined, and may either be given by some finite number above 1 or by $\infty$.

(ii) Fix any $\gamma \in(1, \chi)$. If $a_{j} \in\left(1, a_{j}^{u}(\gamma)\right)$, the difference $\delta_{j+1}^{\alpha R}-\delta_{j}^{\alpha R}$ is large enough to violate (32) for $\alpha^{\prime}=\gamma$ and small enough to satisfy (32) for $\alpha^{\prime}=1$. Moreover, $\delta_{j+1}^{\alpha R}-\delta_{j}^{\alpha R}$ is strictly increasing in $\alpha^{\prime}$ for all $\alpha^{\prime}>1$ by Lemma 10 (iii) and (i). Consequently, there exists a unique threshold $\bar{\beta}_{j} \in(1, \gamma)$ such that the downward IC constraint is violated for all $\alpha^{\prime} \in\left(\bar{\beta}_{j}, \chi\right)$, and satisfied for all $\alpha^{\prime} \in\left[0, \bar{\beta}_{j}\right]$.

Lemma 13. Consider any $j \in J_{-n}$. If $a_{j}<a_{j}^{0}$, there is a continuously differentiable and strictly increasing function $\beta_{j}^{D}:[0, \chi] \rightarrow(0, \chi)$ such that $\left(c^{\alpha R}, y^{\alpha R}\right)$ satisfies the downward IC constraint if and only if $\alpha_{j+1} \geq \beta_{j}^{D}\left(\alpha_{j}\right)$. Function $\beta_{j}^{D}$ has a unique fixed point at $\underline{\beta}_{j} \in(0,1)$, where $\frac{d \beta_{j}^{D}\left(\underline{\beta}_{j}\right)}{d \alpha_{j}}<1$.

Proof. Consider some $j \in J_{-n}$ and some $a_{j} \in\left(1, a_{j}^{0}\right)$, where $a_{j}^{0}$ is defined in Lemma 11 (i). Hence, there is a number $\underline{\beta}_{j} \in(0,1)$ such that $\left(c^{\alpha R}, y^{\alpha R}\right)$ satisfies the downward $\overline{\mathrm{IC}}$ constraint 26) if $\alpha_{j}=\alpha_{j+1} \geq \underline{\beta}_{j}$, and violates (26) if $\alpha_{j}=\alpha_{j+1}<\underline{\beta}_{j}$. Recall that $\delta_{k}^{\alpha R}$ is strictly increasing in $\alpha_{k}$ for each $k \in J$ and each $\alpha_{k} \in[0, \chi)$ by Lemma 9 and that changes in $\alpha_{j}$ and $\alpha_{j+1}$ only affect the left-hand side of the downward IC 26).

First, fix some $\alpha_{j}=x \in\left(\underline{\beta}_{j}, \chi\right)$. For $\alpha_{j+1}=\underline{\beta}_{j}$, the difference $\delta_{j+1}^{\alpha R}-\delta_{j}^{\alpha R}$ is smaller than for $\alpha_{j}=\alpha_{j+1}=\underline{\beta}_{j}$, and (26) is violated. For $\alpha_{j+1}=\alpha_{j}=x$, in contrast, 26] is satisfied by Lemma 11. As $\delta_{j+1}^{\alpha R}$ is increasing in $\alpha_{j+1}$, there is a unique number $\beta_{1}^{D}(x) \in$ $(\underline{\beta}, x)$ such that 26$)$ is satisfied for all $\alpha_{j+1} \geq \beta_{j}^{D}(x)$, and violated for all $\alpha_{j+1}<\beta_{j}^{D}(x)$. 
Second, fix some $\alpha_{j}=x \in\left[0, \underline{\beta}_{j}\right)$. For $\alpha_{j+1}=\underline{\beta}$, the difference $\delta_{j+1}^{\alpha R}-\delta_{j}^{\alpha R}$ is larger than for $\alpha_{j}=\alpha_{j+1}=\underline{\beta}_{j}$ so that 26) is satisfied. For $\alpha_{j+1}=x$, in contrast, 26) is violated by Lemma 11 . By the monotonicity of $\beta_{j+1}^{\alpha R}$ in $\alpha_{j+1}$, there is a unique number $\beta_{j}^{D}(x) \in\left(x, \underline{\beta}_{j}\right)$ such that 26 is satisfied if $\alpha_{j+1} \geq \beta_{j}^{D}(x)$, and violated if $\alpha_{j+1}<\beta_{j}^{D}(x)$. This also implies that Hence, $\beta_{1}^{D}(x) \in(0, \chi)$ for all $x \in[0, \chi)$.

To prove that $\beta_{j}^{D}$ is continuously differentiable and strictly increasing, note that $\beta_{j}^{D}\left(\alpha_{j}\right)$ is implicitly defined by

$$
\delta_{j+1}^{\alpha R}\left(\beta_{j}^{D}\left(\alpha_{j}\right)\right)-\delta_{j}^{\alpha R}\left(\alpha_{j}\right)=h\left(y_{j}^{\alpha R}, \omega_{j}\right)-h\left(y_{j}^{\alpha R}, \omega_{j+1}\right) .
$$

The right-hand side of this equation is constant in $\alpha_{j}$ and $\alpha_{j+1}$. The left-hand side is continuously differentiable and monotonic in both weights as long as these are below $\chi$. Hence, $\beta_{j}^{D}$ is continuously differentiable in $\alpha_{j}$. Using the implicit function theorem, the derivative is given by

$$
\frac{d \beta_{j}^{D}\left(\alpha_{j}\right)}{d \alpha_{j}}=\frac{\frac{d \delta_{j}^{\alpha R}\left(\alpha_{j}\right)}{d \alpha_{j}}}{\frac{d \delta_{j+1}^{\alpha R}\left(\beta_{j}^{D}\left(\alpha_{j}\right)\right)}{d \alpha_{j+1}}}>0,
$$

where the numerator and the denominator are strictly positive by Lemma 9 .

Finally, we know from Lemma 11 that $\beta_{j}^{D}$ has a unique fixed point at $\underline{\beta}_{j}<1$ whenever $a_{j} \in\left(1, a_{j}^{0}\right)$. As the downward IC constraint is satisfied at this fixed point, Lemma 10 (ii) ensures that $\frac{d \delta_{j}^{\alpha R}\left(\underline{\beta}_{j}\right)}{d \alpha_{j}}<\frac{d \delta_{j+1}^{\alpha R}(\underline{\beta})}{d \alpha_{j+1}}$. Hence, $\frac{d \beta_{j}^{D}\left(\underline{\beta}_{j}\right)}{d \alpha_{j}}<1$.

Lemma 14. Consider any $j \in J_{-n}$. If $a_{j}<a_{j}^{u}(\gamma)$ for some $\gamma \in(1, \chi)$, there is a continuously differentiable and strictly increasing function $\beta_{j}^{U}:[0, \chi) \rightarrow\left(0, \chi_{n}\right)$ such that $\left(c^{\alpha R}, y^{\alpha R}\right)$ violates the upward IC constraint if and only if $\alpha_{j+1}>\beta_{j}^{U}\left(\alpha_{j}\right)$. Function $\beta_{j}^{U}$ has a unique fixed point at $\bar{\beta}_{j} \in(1, \chi)$, where $\frac{d \beta_{j}^{U}\left(\underline{\beta}_{j}\right)}{d \alpha_{j}}<1$.

Proof. The proof of Lemma 14 follows the same steps as the proof of Lemma 13 . Consider some $j \in J_{-n}$ and some $a_{j}$ between 1 and the treshold $a_{j}^{u}(\gamma)$ for some $\gamma \in(1, \chi)$, as defined in Lemma 12 (ii). Hence, there is a number $\bar{\beta}_{j} \in(1, \gamma)$ such that $\left(c^{\alpha R}, y^{\alpha R}\right)$ satisfies the upward IC constraint (32) if $\alpha_{j}=\alpha_{j+1} \geq \bar{\beta}_{j}$, and violates (32) if $\alpha_{j}=\alpha_{j+1}<\bar{\beta}_{j}$.

First, fix some $\alpha_{j}=x \in\left(\bar{\beta}_{j}, \chi\right)$. The upward IC constraint is satisfied for $\alpha_{j+1}=\bar{\beta}_{j}$, and violated $\alpha_{j+1}=x$ by Lemma 12 . By the monotonicity of $\delta_{j+1}^{\alpha R}$ in $\alpha_{j+1}$, there is a unique number $\beta_{1}^{U}(x) \in\left(\bar{\beta}_{j}, x\right)$ such that $(32)$ is satisfied if $\alpha_{j+1} \leq \beta_{j}^{U}(x)$, and violated if $\alpha_{j+1}>\beta_{j}^{U}(x)$.

Second, fix some $\alpha_{j}=x \in\left[0, \bar{\beta}_{j}\right)$. The upward IC constraint is violated for $\alpha_{j+1}=\bar{\beta}_{j}$, and satisfied for $\alpha_{j+1}=x$ by Lemma 12 . Hence, there is a unique number $\beta_{j}^{U}(x) \in\left(x, \bar{\beta}_{j}\right)$ such that 32 is satisfied if $\alpha_{j+1} \leq \beta_{j}^{U}(x)$ and violated if $\alpha_{j+1}>\beta_{j}^{U}(x)$. Hence, we also have $\beta_{j}^{U}(x) \in(0, \chi)$ for all $x \in[0, \chi)$. 
For each $\alpha_{j} \in[0, \chi), \beta_{j}^{U}\left(\alpha_{j}\right)$ is implicitly defined by

$$
\delta_{j+1}^{\alpha R}\left(\beta_{j}^{U}\left(\alpha_{j}\right)\right)-\delta_{j}^{\alpha R}\left(\alpha_{j}\right)=h\left(y_{j+1}^{\alpha R}, \omega_{j}\right)-h\left(y_{j+1}^{\alpha R}, \omega_{j+1}\right),
$$

where the left-hand side is continuously differentiable and monotonic in $\alpha_{j}$ and $\alpha_{j+1}$, and the right-hand side is constant in both social weights. Using the implicit function theorem, the derivative of $\beta_{j}^{U}$ with respect to $\alpha_{j}$ is given by

$$
\frac{d \beta_{j}^{U}\left(\alpha_{j}\right)}{d \alpha_{j}}=\frac{\frac{d \delta_{j}^{\alpha R}\left(\alpha_{j}\right)}{d \alpha_{j}}}{\frac{d \delta_{j+1}^{\alpha R}\left(\beta_{j}^{U}\left(\alpha_{j}\right)\right)}{d \alpha_{j+1}}}>0,
$$

where the numerator and the denominator are strictly positive by Lemma 9 . This derivative is continuous and strictly positive for all $\alpha_{1} \in[0, \chi)$.

Finally, Lemma 12 implies that $\beta_{j}^{U}$ has a unique fixed point at $\bar{\beta}_{j} \in(1, \chi)$ whenever $a_{j} \in\left(1, a_{j}^{U}(\gamma)\right)$ for some $\gamma \in(1, \chi)$. At this fixed point, the downward IC constraint is satisfied and $\frac{d \delta_{j}^{\alpha R}\left(\bar{\beta}_{j}\right)}{d \alpha_{j}}<\frac{d \delta_{j+1}^{\alpha R}\left(\bar{\beta}_{j}\right)}{d \alpha_{j+1}}$ by Lemma 10 (i). Consequently, $\frac{d \beta_{j}^{U}\left(\bar{\beta}_{j}\right)}{d \alpha_{j}}<1$.

Finally, this allows us to prove Lemma 5.

Proof. Define $a_{j}^{U s}:=\sup \left\{a_{j}^{U}(\gamma) \mid \gamma \in(1, \chi)\right\}$. For all $a_{j}<\min \left\{a_{j}^{0}, a_{j}^{U s}\right\}$, Lemmas 13 and 14 directly ensure the existence of two functions $\beta_{j}^{D}$ and $\beta_{j}^{U}$ with the properties stated in Lemma 5 ,

It only remains to prove that $\beta_{j}^{U}\left(\alpha_{j}\right)>\beta_{j}^{D}\left(\alpha_{j}\right)$ for all $\alpha_{j} \in[0, \chi)$. Note that $h\left(y_{j+1}^{\alpha R}, \omega_{j}\right)-h\left(y_{j+1}^{\alpha R}, \omega_{j+1}\right)>h\left(y_{j}^{\alpha R}, \omega_{j}\right)-h\left(y_{j}^{\alpha R}, \omega_{j+1}\right)$ by the properties of the effort cost function $h$. Hence, equations (35) and (37) imply that $\delta_{j+1}^{\alpha R}\left(\beta_{j}^{U}(x)\right)>\delta_{j+1}^{\alpha R}\left(\beta_{j}^{D}(x)\right)$. Because $\delta_{j+1}^{\alpha R}$ is strictly increasing in $\alpha_{j+1}$ by Lemma 9, we have $\delta_{j}^{U}(x)>\delta_{j}^{D}(x)$ for all $x \in[0, \chi)$.

\section{Proof of Proposition 1}

I prove Proposition 1 through Lemmas 15 to 20 , which identify properties of the optimal allocation $\left(c^{\alpha}, y^{\alpha}\right)$ for different sets of binding IC constraints along the skill dimension. To refer to these different constellations, I will henceforth say that two skill groups $j$ and $k$ are downwards-linked (upwards-linked) if all downward (upward) IC constraints between the pairs $(j, j+1), \ldots,(k-1, k)$ are binding.

Lemma 15. For each $j \in J$, output $y_{j}^{\alpha}$ and participation threshold $\delta_{j}^{\alpha}$ satisfy the conditions

$$
\begin{aligned}
\delta_{j}^{\alpha} & =y_{j}^{\alpha}-h\left(y_{j}^{\alpha}, \omega_{j}\right)+\frac{\alpha_{j}-1}{A_{j}\left(\delta_{j}^{\alpha}\right)}+\frac{\nu_{j-1}^{D}-\nu_{j}^{D}-\nu_{j-1}^{U}+\nu_{j}^{U}}{f_{j} g_{j}\left(\delta_{j}^{\alpha}\right)}, \\
1-h_{y}\left(y_{j}, \omega_{j}\right) & =\frac{h_{y}\left(y_{j}, \omega_{j}\right)-h_{y}\left(y_{j}, \omega_{j+1}\right)}{f_{j} G_{j}\left(\delta_{j}^{\alpha}\right)} \nu_{j}^{D}-\frac{h_{y}\left(y_{j}, \omega_{j-1}\right)-h_{y}\left(y_{j}, \omega_{j}\right)}{f_{j} G_{j}\left(\delta_{j}^{\alpha}\right)} \nu_{j-1}^{U},
\end{aligned}
$$


where $\nu_{k}^{D}$ and $\nu_{k}^{U}$ denote the Lagrange multipliers associated with the downward IC 16) and the upward IC (17), respectively, between the workers with skill types $\omega_{k}$ and $\omega_{k+1}$.

Proof. The Lagrangian of the optimal tax problem is given by

$$
\begin{aligned}
\mathcal{L}= & \sum_{j=1}^{n} f_{j}\left(\int_{\underline{\delta}}^{\delta_{j}} g_{j}(\delta) \gamma(\omega, \delta) \Psi\left[c_{j}-h\left(y_{j}, \omega_{j}\right)-\delta\right] d \delta+\int_{\delta_{j}}^{\bar{\delta}} g_{j}(\delta) \gamma(\omega, \delta) \Psi\left(c_{0}\right) d \delta\right) \\
& +\lambda\left[\sum_{j=1}^{n} f_{j} G_{j}\left(\delta_{j}\right)\left(y_{j}-c_{j}+c_{0}\right)-c_{0}\right] \\
& +\sum_{j=1}^{n-1} \nu_{j}^{D}\left[c_{j+1}-h\left(y_{j+1}, \omega_{j+1}\right)-c_{j}+h\left(y_{j}, \omega_{j+1}\right)\right] \\
& +\sum_{j=1}^{n-1} \nu_{j}^{U}\left[c_{j}-h\left(y_{j}, \omega_{j}\right)-c_{j+1}+h\left(y_{j+1}, \omega_{j}\right)\right] .
\end{aligned}
$$

For any $j \in\{2, \ldots, n-1\}$, the FOCs with respect to $c_{j}$ and $y_{j}$ are given by:

$$
\begin{array}{rlr}
\mathcal{L}_{c_{j}}= & f_{j}\left[G_{j}\left(\delta_{j}^{\alpha R}\right)\left(\alpha_{j}-\lambda\right)+\lambda g_{j}\left(\delta_{j}^{\alpha R}\right)\left(y_{j}^{\alpha R}-c_{j}^{\alpha R}+c_{0}^{\alpha R}\right)\right]+\nu_{j-1}^{D}-\nu_{j}^{D}-\nu_{j-1}^{U}+\nu_{j}^{U} & \stackrel{!}{=} 0 \\
\mathcal{L}_{y_{j}}= & f_{j}\left[-h_{y}\left(y_{j}^{\alpha R}, \omega_{j}\right)\left(G_{j}\left(\delta_{j}^{\alpha R}\right) \alpha_{j}+\lambda g_{j}\left(\delta_{j}^{\alpha R}\right)\left(y_{j}^{\alpha R}-c_{j}^{\alpha R}+c_{0}^{\alpha R}\right)\right)+\lambda G_{j}\left(\delta_{j}^{\alpha R}\right)\right] & \\
& -\left(\nu_{j-1}^{D}+\nu_{j}^{U}\right) h_{y}\left(y_{j}^{\alpha}, \omega_{j}\right)+\nu_{j}^{D} h_{y}\left(y_{j}^{\alpha}, \omega_{j+1}\right)+\nu_{j-1}^{U} h_{y}\left(y_{j}^{\alpha}, \omega_{j-1}\right) & \stackrel{!}{=} 0
\end{array}
$$

The FOC with respect to $c_{0}$ is identical to the one for the relaxed problem (see Lemma 7). Combining the FOCs with respect to $c_{j}$ for all $j \in\{0,1, \ldots, n\}$ gives $\lambda=1$. Equation 39 then follows from rearranging the FOC with respect to $c_{j}$. Combining the FOCs with respect $c_{j}$ and $y_{j}$ gives equation 40 .

Below, I will exploit that, setting $\nu_{j}^{U}=\nu_{j}^{D}=0$, Lemma 15 also provides the conditions for the solution to a relaxed problem that ignores the local IC constraints between the workers with skill types $j$ and $j+1$.

Lemma 16. For any $\alpha \in \mathcal{A}^{\chi}$, if $k$ is the lowest skill group that is upwards-linked with $l>k$, then

(i) $\alpha_{k}>1$,

(ii) $\delta_{j}^{\alpha}>y_{j}^{\alpha}-h\left(y_{j}^{\alpha}, \omega_{j}\right)$ for all $j \in\{k, \ldots, l\}$, and

(iii) $\alpha_{j}>1$ for all $j \in\{1, \ldots, l\}$.

Proof. (i) First, note that $k$ and $l>k$ cannot be upwards-linked unless either $\alpha_{j+1}>$ $\alpha_{j}\left(\beta_{j}^{U}\right)$ for at least one $j \in\{k, \ldots, l-1\}$ or the downward IC between $l$ and $l+1$ is binding, $\nu_{l}^{D}>0$. Assume both conditions would be violated, and consider a relaxed problem where all local ICs between $k$ and $l$ are ignored. In the solution to this problem, $\delta_{k} \geq \delta_{k}^{\alpha R}, \delta_{j}=\delta_{j}^{\alpha R}$ for all $j \in\{k+1, \ldots, l\}$ and $y_{j}=y_{j}^{\alpha R}$ for all 
$j \in\{k, \ldots, l\}$. Hence, the solution to this relaxed problem satisfies all upward IC constraints between $k$ and $l$. Consequently, these upward IC constraints cannot be binding in $\left(c^{\alpha}, y^{\alpha}\right)$.

Second, assume that there is a skill group $j \in\{k, \ldots, l-1\}$ such that $\alpha_{j+1}>\alpha_{j}\left(\beta_{j}^{U}\right)$. By Lemma 5, $\alpha_{j}>\bar{\beta}_{j}>1$. For the monotonicity of $\alpha \in \mathcal{A}$, this ensures that $\alpha_{k}>1$. Third, assume that the upward IC between $l-1$ and $l$ as well as the downward IC between $l$ and $l+1$ are binding and that $\alpha_{j+1} \leq \alpha_{j}\left(\beta_{j}^{U}\right)$ for all $j \in\{k, \ldots, l-1\}$. Consider a relaxed problem where only the local ICs between skill groups $l-1$ and $l$ are ignored. In the solution to this relaxed problem, the downward IC between $l$ and $l+1$ has to be binding as well. From Lemma 15, the solution of this problem satisfies $y_{l-1}=y_{l-1}^{\alpha R}, \delta_{l-1} \geq \delta^{*}\left(\omega_{l-1}\right)+\left(\alpha_{l-1}-1\right) / A_{l-1}\left(\delta_{l-1}\right)$ and $\delta_{l}<y_{l}-h\left(y_{l}, \omega_{l}\right)+$ $\left(\alpha_{l}-1\right) / A_{l}\left(\delta_{l}\right)$. By $\alpha_{l}<\alpha_{l-1}$, this allocation has to satisfy

$$
\delta_{l}-\delta_{l-1}<\left(\frac{1}{A_{l}\left(\delta_{l}\right)}-\frac{1}{A_{l-1}\left(\delta_{l-1}\right)}\right)\left(\alpha_{l-1}-1\right)+y_{l}-h\left(y_{l}, \omega_{l}\right)-\delta^{*}\left(\omega_{l-1}\right) .
$$

The allocation only violates the upward IC constraint (32) if $\delta_{l}-\delta_{l-1}>h\left(y_{l}, \omega_{l-1}\right)-$ $h\left(y_{l}, \omega_{l}\right)$. Hence, this can only result if

$$
\left(\frac{1}{A_{l}\left(\delta_{l}^{\alpha P}\right)}-\frac{1}{A_{l-1}\left(\delta_{l-1}^{\alpha P}\right)}\right)\left(\alpha_{l-1}-1\right)>\delta^{*}\left(\omega_{l-1}\right)-\left[y_{l}^{\alpha P}-h\left(y_{l}^{\alpha P}, \omega_{l-1}\right)\right] \geq 0,
$$

where the last inequality follows by the definition of $\delta^{*}\left(\omega_{l-1}\right)$. Recall that $A_{l}\left(\delta_{l}^{\alpha P}\right)<$ $A_{l-1}\left(\delta_{l-1}^{\alpha P}\right)$ by Lemma 2 . Hence, the condition above can only be satisfied if $\alpha_{l-1}$ is strictly larger than 1. But this implies that $\alpha_{k}>1$ for all $\alpha \in \mathcal{A}^{\chi}$.

(ii) By construction, $k$ is the lowest skill group that is upwards-linked to $l$, i.e., the upward IC between $k-1$ and $k$ is not binding. Hence, $\delta_{k}^{\alpha}>y_{k}^{\alpha}-h\left(y_{k}^{\alpha}, \omega_{k}\right)+$ $\frac{\alpha_{k}-1}{A_{k}\left(\delta_{k}^{\alpha}\right)}>y_{k}^{\alpha}-h\left(y_{k}^{\alpha}, \omega_{k}\right)$ because $\alpha_{k}>1$ as shown in part (i). By Lemma 15 , we have $y_{j}^{\alpha}>y_{j}^{\alpha R}$ for all $j \in\{k, \ldots, l-1\}$ because the corresponding upward IC constraints are binding, i.e., $\nu_{j}^{U}>0$. As usual, incentive compatibility also requires $y_{j+1}^{\alpha}>y_{j}^{\alpha}$. The single-crossing condition hence ensures that, for all $j \in\{k, \ldots, l-1\}$, $y_{j}^{\alpha}-h\left(y_{j}^{\alpha}, \omega_{j}\right)>y_{j+1}^{\alpha}-h\left(y_{j+1}^{\alpha}, \omega_{j}\right)$ and

$$
\begin{aligned}
\delta_{j+1}^{\alpha} & =\delta_{j}^{\alpha}+h\left(y_{j+1}^{\alpha}, \omega_{j}\right)-h\left(y_{j+1}^{\alpha}, \omega_{j+1}\right) \\
& >y_{j}^{\alpha}-h\left(y_{j}^{\alpha}, \omega_{j}\right)+h\left(y_{j+1}^{\alpha}, \omega_{j}\right)-h\left(y_{j+1}^{\alpha}, \omega_{j+1}\right) \geq y_{j+1}^{\alpha}-h\left(y_{j+1}^{\alpha}, \omega_{j+1}\right) .
\end{aligned}
$$

(iii) Assume that $m$ is the highest skill group that is upwards-linked with $k$. By the previous arguments, $\delta_{m}^{\alpha}>y_{m}^{\alpha}-h\left(y_{m}^{\alpha}, \omega_{m}\right)$. At the same time, $\delta_{m}^{\alpha}<y_{m}^{\alpha}-h\left(y_{m}^{\alpha}, \omega_{m}\right)+$ $\left(\alpha_{m}-1\right) / A_{m}\left(\delta_{m}^{\alpha}\right)$ by equation $(39)$. Both statements can only be consistent if $\alpha_{m}>1$. For all $\alpha \in \mathcal{A}^{\chi}$, we hence have $\alpha_{j}>1$ for all $j \in\{1, \ldots, m\}$. 
Lemma 17. For any $\alpha \in \mathcal{A}^{\chi}$, if the skill groups $j$ and $j+1$ are downwards-linked and $\delta_{j}^{\alpha} \leq y_{j}^{\alpha}-h\left(y_{j}^{\alpha}, \omega_{j}\right)$, then $\left(c^{\alpha}, y^{\alpha}\right)$ also involves $\delta_{j+1}^{\alpha} \leq y_{j+1}^{\alpha}-h\left(y_{j+1}^{\alpha}, \omega_{j}\right)$.

Proof. If the downward IC constraint (26) is binding, $\delta_{j+1}=\delta_{j}+h\left(y_{j}, \omega_{j}\right)-h\left(y_{j}, \omega_{j+1}\right)$. Hence, we have $\delta_{j+1} \leq y_{j}^{\alpha}-h\left(y_{j}^{\alpha}, \omega_{j+1}\right) \leq y_{j+1}^{\alpha}-h\left(y_{j+1}^{\alpha}, \omega_{j+1}\right)$. The second inequality results because $y_{j} \leq y_{j+1}$ in every incentive-compatible allocation and $y_{j+1}^{\alpha} \leq \arg \max _{y>0} y-$ $h\left(y, \omega_{j+1}\right)$ if the skill groups $j$ and $j+1$ are downwards-linked.

Lemma 18. For any $\alpha \in \mathcal{A}^{\chi}$, there exists a number $k^{\alpha} \in(0, n]$ such that labor supply in skill group $j$ is upwards distorted at the extensive margin if and only if $j \in\left\{l \in J: l \leq k^{\alpha}\right\}$.

Proof. Fix some $\alpha \in \mathcal{A}^{\chi}$. Consider a skill group $j$ for which labor supply in $\left(c^{\alpha}, y^{\alpha}\right)$ is not upwards distorted at the extensive margin, $\delta_{j}^{\alpha} \leq y_{j}^{\alpha}-h\left(y_{j}^{\alpha}, \omega_{j}\right)$. As I will show, this ensures that optimal labor supply is not upwards distorted at the extensive margin in any skill group $h>j$ as well.

By Lemma 16, $j$ cannot be upwards-linked to other skill groups. Let $l$ be the highest skill group to which $j$ is downwards-linked. (Note that $l$ equals $j$ if the downward IC between skill groups $j$ and $j+1$ is slack.) By Lemma 17, $\delta_{k}^{\alpha} \leq y_{k}^{\alpha}-h\left(y_{k}^{\alpha}, \omega_{k}\right)$ for all $k \in\{j, \ldots, l\}$. By Lemma 15, we must at the same time have $\delta_{l}^{\alpha} \geq y_{l}^{\alpha}-h\left(y_{l}^{\alpha}, \omega_{l}\right)+$ $\left(\alpha_{l}-1\right) / A_{l}\left(\delta_{l}^{\alpha}\right)$. Hence, we must have $\alpha_{l} \leq 1$.

Consequently, there cannot be upwards-linked skill groups above $l$ by Lemma 15. For any unlinked skill group $k>l, \alpha_{k}<\alpha_{l} \leq 1$ ensures that labor supply is not upwards distorted at the extensive margin. For any downwards-linked skill groups $k>l$ and $m$, $\alpha_{k}<1$ and $\nu_{k}^{D}>0$ jointly ensure that $\delta_{k}<y_{k}^{\alpha}-h\left(y_{k}^{\alpha}, \omega_{k}\right)$. By Lemma 17, the same conditions holds for the skill groups $k+1$ to $m$. Hence, labor supply is not upwards distorted at the extensive margin in any skill group $h>j$.

Lemma 19. Consider some weight $\alpha \in \mathcal{A}^{\chi}$ such that the optimal tax problem has an interior solution $\left(c^{\alpha}, y^{\alpha}\right)$. Then,

(i) the consumption level $c_{0}^{\alpha}$ of the unemployed is strictly positive;

(ii) there is a number $k^{\alpha} \in(0, n)$ such that optimal output is

(a) upwards distorted at the extensive margin in skill group $j$ if and only if $j \leq k^{\alpha}$, and

(b) downwards distorted or undistorted at the intensive margin in skill group $j$ for all $j \geq k^{\alpha}$;

(iii) optimal output in the highest skill group $n$ is undistorted at the intensive margin and downwards distorted at the extensive margin.

Proof. (i) Consider some implementable and Pareto-efficient allocation $(c, y)$ with $c_{0} \leq$ 0 . In this allocation, the feasibility condition must hold with equality, so that $c_{0}=$ 
$\sum_{j=1}^{n} f_{j} G_{j}\left(\delta_{j}\right)\left(y_{j}-c_{j}+c_{0}\right) \leq 0$. Moreover, an increase in $c_{0}$ must not self-financing. I will show that there exists a marginal variation that is feasible, incentive-compatible and welfare-increasing given any weight sequence $\alpha \in \mathcal{A}^{\chi}$.

Consider an allocation $(\tilde{c}, y)$ with $\tilde{c}_{j}=c_{j}-\varepsilon$ for all $j \in J$ and $\tilde{c}_{0}$ chosen to balance the feasibility condition. In particular, consider a marginal increase in $\varepsilon$ from $\varepsilon=0$. This leaves the IC constraints between all workers satisfied, but induces some workers in all skill groups to leave the labor force. Taking these responses into account, we have

$$
\left.\frac{d \tilde{c}_{0}}{d \varepsilon}\right|_{\varepsilon=0}=\frac{\sum_{j=1}^{n} f_{j} G_{j}\left(\delta_{j}\right)-\sum_{j=1}^{n} f_{j} G_{j}\left(\delta_{j}\right) Z_{j}}{1-\sum_{j=1}^{n} f_{j} G_{j}\left(\delta_{j}\right)+\sum_{j=1}^{n} f_{j} G_{j}\left(\delta_{j}\right) Z_{j}}
$$

where the denominator is strictly positive (otherwise, an increase in $c_{0}$ would be selffinancing) and $Z_{j}=\frac{g_{j}\left(\delta_{j}\right)}{G_{j}\left(\delta_{j}\right)}\left(y_{j}-c_{j}+c_{0}\right)=\frac{g_{j}\left(\delta_{j}\right)}{G_{j}\left(\delta_{j}\right)}\left(y_{j}-h\left(y_{j}, \omega_{j}\right)-\delta_{j}\right)$. If allocation $(c, y)$ is welfare-optimal, there is a unique number $k \in(0, n]$ such that $Z_{j}<0$ for all $j<k$ and $Z_{j} \geq 0$ for all $j \geq k$ by Lemma 17. Moreover, $\frac{g_{j}\left(\delta_{j}\right)}{G_{j}\left(\delta_{j}\right)}=A_{j}\left(\delta_{j}\right)>A_{k}\left(\delta_{k}\right)$ for all $j<k$ and $\frac{g_{j}\left(\delta_{j}\right)}{G_{j}\left(\delta_{j}\right)}<A_{k}\left(\delta_{k}\right)$ for all $j>k$ by Lemma 2. Hence, we have

$$
\sum_{j=1}^{n} f_{j} G_{j}\left(\delta_{j}\right) Z_{j}<A_{k}\left(\delta_{k}\right) \sum_{j=1}^{n} f_{j} G_{j}\left(\delta_{j}\right)\left(y_{j}-c_{j}+c_{0}\right)=A_{k}\left(\delta_{k}\right) c_{0} \leq 0,
$$

which implies that $\left.\frac{d \tilde{c}_{0}}{d \varepsilon}\right|_{\varepsilon=0}>\frac{\sum_{j=1}^{n} f_{j} G_{j}\left(\delta_{j}\right)}{1-\sum_{j=1}^{n} f_{j} G_{j}\left(\delta_{j}\right)}$. The marginal welfare effect of increasing $\varepsilon$ follows as

$$
\begin{aligned}
\left.\frac{d W(c, y ; \alpha)}{d \varepsilon}\right|_{\varepsilon=0} & =-\sum_{j=1}^{n} f_{j} G_{j}\left(\delta_{j}\right) \alpha_{j}+\left.\left[1-\sum_{j=1}^{n} f_{j} G_{j}\left(\delta_{j}\right)\right] \alpha_{0} \frac{d \tilde{c}_{0}}{d \varepsilon}\right|_{\varepsilon=0} \\
& >-\sum_{j=1}^{n} f_{j} G_{j}\left(\delta_{j}\right) \alpha_{j}+\sum_{j=1}^{n} f_{j} G_{j}\left(\delta_{j}\right) \alpha_{0}=\alpha_{0}-\alpha_{M},
\end{aligned}
$$

where $\alpha_{M}=1$ is the average social weight in the population. For all $\alpha \in \mathcal{A}^{\chi}$, $\alpha_{0}>\alpha_{M}$. Hence, an increase in $\varepsilon$ is strictly welfare-increasing, and the initial allocation with $c_{0} \leq 0$ is not welfare-maximizing.

(ii) For part (a), there exists a number $k^{\alpha} \in(0, n]$ with the required properties by Lemma 18. Assume that $k^{\alpha}=n$, i.e., that labor supply in all skill groups is upwards distorted at the extensive margin. The feasibility condition then requires that $c_{0}=$ $\sum_{j=1}^{n} f_{j} G_{j}\left(\delta_{j}^{\alpha}\right)\left(y_{j}^{\alpha}-c_{j}^{\alpha}+c_{0}^{\alpha}\right)=\sum_{j=1}^{n} f_{j} G_{j}\left(\delta_{j}^{\alpha}\right)\left(y_{j}^{\alpha}-h\left(y_{j}^{\alpha}, \omega_{j}\right)-\delta_{j}^{\alpha}\right)<0$. This is inconsistent with part (i) of this Lemma. Hence, $k^{\alpha}<n$ for all $\alpha \in \mathcal{A}^{\chi}$.

For the statement in part (b), note that labor supply in skill group $j$ can only be upwards distorted at the intensive margin if $j-1$ and $j$ are upwards-linked, i.e., $\nu_{j-1}^{U}>0$ (see Lemma 15). By Lemma 16 (ii), this can only be true if labor supply 
in skill group $j$ is upwards distorted at the extensive margin.

(iii) For all $\alpha \in \mathcal{A}^{\chi}, \delta_{n}^{\alpha}<y_{n}^{\alpha}-h\left(y_{n}^{\alpha}, \omega_{j}\right)$ as argued in the proof to part (iii). Hence, $n-1$ and $n$ cannot be upwards-linked by Lemma 16. If $n-1$ and $n$ are downwards-linked or unlinked, $y_{n}^{\alpha}=y_{n}^{\alpha R}$ : Labor supply in skill group $n$ is undistorted at the intensive margin. This moreover implies that $y_{n}^{\alpha}-h\left(y_{n}^{\alpha}, \omega_{n}\right)=\delta^{*}(n)$. Hence, $\delta_{n}^{\alpha}<\delta^{*}\left(\omega_{n}\right)$ : labor supply in skill group is downwards distorted at the extensive margin.

To prove Proposition 1, it only remains to show that the optimal tax problem has a solution with $\delta_{j} \in[\underline{\delta}, \bar{\delta})$ for any $\alpha \in \mathcal{A}^{\chi}$.

Lemma 20. For all $\alpha \in \mathcal{A}^{\chi}$, the optimal tax problem has a maximum $\left(c^{\alpha}, y^{\alpha}\right)$ with $\delta_{j} \in[\underline{\delta}, \bar{\delta})$.

Proof. Fix some $\alpha \in \mathcal{A}^{\chi}$. By Lemma 3, the FOCs with respect to $y_{j}$ and $\delta_{j}$ are satisfied by a unique tuple $\left(y_{j}^{\alpha}, \delta_{j}^{\alpha}\right)$ if the local IC constraints between skill groups $j, j-1$ and $j+1$ are non-binding. In the following, I only consider the cases where skill group $j$ is either downwards-linked or upwards-linked to skill group $j+1$ only. Similar proofs are available on request for cases where some skill groups $k$ and $l>k+1$ are downwards-linked or upwards-linked.

First, assume that the downward IC (26) between skill groups $j$ and $j+1$ is binding. For simplicity, I henceforth write $H_{j}\left(y_{j}\right):=h\left(y_{j}, \omega_{j}\right)-h\left(y_{j}, \omega_{j+1}\right)$. The FOCs with respect to $c_{j}, c_{j+1}$ and $y_{j}$ (see Lemma 15) can be combined to get the optimality conditions

$$
\begin{aligned}
& Z_{1}\left(y_{j}, \delta_{j}\right)=B_{j}\left(\delta_{j}, y_{j}\right)+B_{j+1}\left(\delta_{j+1}, y_{j+1}\right) \quad=0 \\
& Z_{2}\left(y_{j}, \delta_{j}\right)=B_{j+1}\left(\delta_{j+1}, y_{j+1}\right)+f_{j} G_{j}\left(\delta_{j}\right) C_{j}\left(y_{j}\right)=0 \text {, }
\end{aligned}
$$

where $B_{k}\left(\delta_{k}, y_{k}\right):=f_{k}\left[G_{k}\left(\delta_{k}\right)\left(\alpha_{k}-1\right)+g_{k}\left(\delta_{k}\right)\left(y_{k}-h\left(y_{k}, \omega_{k}\right)-\delta_{k}\right)\right]$ and $C_{j}\left(y_{j}\right):=[1-$ $\left.h_{y}\left(y_{j}, \omega_{j}\right)\right] / H_{j}\left(y_{j}\right)$. By the binding downward IC constraint, we have $1-h_{y}\left(y_{j}, \omega_{j}\right)>0$, $C_{j}>0, B_{j+1}<0$ and $B_{j}>0$ in allocation $\left(c^{\alpha}, y^{\alpha}\right) . Z_{1}$ and $Z_{2}$ are continuous in $y_{j}, \delta_{j}$ and $\delta_{j+1}$. Moreover, I will show that $Z_{1}$ has a root in $\delta_{j}$ at which $\partial Z_{1} / \partial \delta_{j}<0$, and that $Z_{2}$ has a root in $y_{j}$ at which $\partial Z_{2} / \partial y_{j}<0$.

To start, consider function $Z_{1}$ for some fixed level $y_{j}<y_{j}^{\alpha R}$. Function $B_{j}\left(\delta_{j}, y_{j}^{\alpha R}\right)$ has a unique root $\delta_{j 1} \in(\underline{\delta}, \bar{\delta})$ for any $\alpha_{k} \in[0, \chi)$ by Lemma 3 . Function $\tilde{B}_{j+1}\left(\delta_{j}\right):=$ $B_{j+1}\left(\delta_{j}+H_{j}\left(y_{j}^{\alpha R}\right), y_{j+1}^{\alpha R}\right)$ either has a unique root $\delta_{j 2}$ in $(\underline{\delta}, \bar{\delta})$ as well, or it is negative for all $\delta_{j} \in \Delta$. In the first case, $\underline{\delta}<\delta_{j 2}<\delta_{j 1}<\bar{\delta}$. By Lemma 3 , $B_{k}$ is strictly decreasing in $\delta_{k}$ at its roots for any $k \in J$. For any $y_{j}<y_{j}^{\alpha R}$, the root $\delta_{j 2}$ is increased, while the root $\delta_{j 1}$ is decreased. As $B_{j}$ and $B_{j+1}$ are continuous, $Z_{1}$ must have a root in the interval $\left(\delta_{j 2}, \delta_{j 1}\right)$. In the second case, if $\delta_{j}^{\alpha}=\underline{\delta}$ results if and only if $B_{j}\left(\underline{\delta}, y_{j}^{\alpha R}\right)+B_{j+1}\left(\underline{\delta}+H_{j}\left(y_{j}^{\alpha R}\right), y_{j+1}^{\alpha R}\right)<0$.

Next, consider $Z_{2}$ for some fixed $\delta_{j} \in\left(\underline{\delta}, \delta_{j}^{\alpha R}\right)$. At any root of $Z_{2}$ in $y_{j}$, we have $B_{j+1}<0$ and $C_{j}\left(y_{j}\right)>0$. Note that $C_{j}^{\prime}\left(y_{j}\right)=-\frac{h_{y}\left(y_{j}, \omega_{j}\right) H_{j}^{\prime}\left(y_{j}\right)+\left(1-h_{y}\left(y_{j}, \omega_{j}\right)\right) H_{j}^{\prime \prime}\left(y_{j}\right)}{H_{j}^{\prime}\left(y_{j}\right)^{2}}$. By 
$h_{y \omega}<0$ and $h_{y y \omega} \leq 0, C_{j}^{\prime}\left(y_{j}\right)<0$ for all $y_{j} \leq y_{j}^{\alpha R}$. The root $y_{j 1}$ of $B_{j+1}$ must again be located left of $y_{j}^{\alpha R}$, the root of $C_{j}$. Hence, function $Z_{2}$ has a root $y_{j}$ in the interval $\left(y_{j 1}, y_{j}^{\alpha R}\right)$.

Second, assume that the upward IC constraint (32) is binding. Recall that this only results for $\alpha_{j} 1$ and $\alpha_{j+1}>1$. Then, the optimal tuple $\left(\delta_{j}, y_{j+1}\right)$ is implicitly defined by

$$
\begin{aligned}
Z_{3}\left(y_{j+1}, \delta_{j}\right)=B_{j}\left(\delta_{j}, y_{j}\right)+B_{j+1}\left(\delta_{j+1}, y_{j+1}\right) & =0 \\
Z_{4}\left(y_{j+1}, \delta_{j}\right)=B_{j+1}\left(\delta_{j+1}, y_{j+1}\right)+f_{j+1} G_{j+1}\left(\delta_{j+1}\right) C_{j+1}\left(y_{j+1}\right) & =0,
\end{aligned}
$$

where $C_{j+1}\left(y_{j+1}\right):=\left[1-h_{y}\left(y_{j+1}, \omega_{j+1}\right)\right] / H_{j}\left(y_{j+1}\right)$. By the binding upward IC constraint, we have $1-h_{y}\left(y_{j+1}, \omega_{j+1}\right)<0, C_{j+1}\left(y_{j+1}\right)<0, B_{j+1}>0$ and $B_{j}<0$ in this optimum.

Again, I start by showing that $Z_{3}$ has a root in $\delta_{j}$ for some fixed $y_{j+1}>y_{j+1}^{\alpha R}$. Function $B_{j}$ has a unique root $\delta_{j 3} \in\left(\delta^{*}\left(\omega_{j}\right), \lim _{\alpha_{j} \rightarrow \chi} \delta_{j}^{\alpha R}\right)$. For any $y_{j+1}<y_{j+1}^{\alpha R}$, function $\tilde{B}_{j+1}\left(\delta_{j}\right)=B_{j+1}\left(\delta_{j}+H_{j}\left(y_{j+1}\right), y_{j+1}\right)$ has a unique root $\delta_{j 4}$ such that $\delta_{j 4}+H_{j}\left(y_{j+1}\right)$ is below the optimal $\delta_{j+1}^{\alpha R}$ for $\alpha_{j+1}<\chi$. Both functions are strictly positive left of these roots, and strictly negative right of these roots. As $B_{j+1}>0$ and $B_{j}<0$ must be satisfied, $\delta_{j 3}<\delta_{j 4}$. Hence, $Z_{3}$ must have a root in $\delta_{j}$ in the interval $\left(\delta_{j 3}, \delta_{j 4}\right)$.

Finally, consider function $Z_{4}$ for some fixed $\delta_{j}>\delta_{j}^{\alpha}$. $B_{j+1}$ has a unique root in $y_{j+1}$ above $y_{j+1}^{\alpha R}$, while $C_{j+1}\left(y_{j+1}\right)$ has a unique root at $y_{j+1}^{\alpha R}$. Both $B_{j+1}$ and $C_{j+1}$ are strictly decreasing at their roots. Hence, $Z_{4}$ has a root in $y_{j+1}$ with $y_{j+1}>y_{j+1}^{\alpha R}$.

\section{Proof of Proposition 2}

Proof. Fix some $\alpha \in \mathcal{A}^{D}$. By Lemma 13 , the relaxed problem's solution $\left(c^{\alpha R}, y^{\alpha R}\right)$ violates the downward IC for at least one pair of skill groups $(k, k+1)$, where $\alpha_{k+1}<\beta_{k}^{D}\left(\alpha_{k}\right)$.

For the first step, consider the intermediate problem $A$ that takes into account the local IC constraints between skill groups $k$ and $k+1$, but ignores the ICs between all other skill pairs. I denote the solution to this problem by $\left(c^{A}, y^{A}\right)$. By $\alpha_{k+1}<\beta_{k}^{D}\left(\alpha_{k}\right)$, the downward IC is binding with $\nu_{k}^{D}>0$. From Lemma 15, we know that $y_{k}^{A}<y_{k}^{\alpha R}, \delta_{k}^{A}<\delta_{k}^{\alpha R}$ and $\delta_{k+1}^{A}>\delta_{k+1}^{\alpha R}$. For all other skill groups $j$, we have $y_{j}^{A}=y_{j}^{\alpha R}$ and $\delta_{j}^{A}=\delta_{j}^{\alpha R}$. In particular, this is true for skill group $k-1$. Hence, $\left(c^{A}, y^{A}\right)$ violates the downward IC constraint between skill groups $k-1$ and $k$, because $\delta_{k}^{\alpha R}-\delta_{k-1}^{\alpha R} \leq h\left(y_{k-1}^{\alpha R}, \omega_{k-1}\right)-h\left(y_{k-1}^{\alpha R}, \omega_{k-1}\right)$ by the construction for any $\alpha \in \mathcal{A}^{D}$.

For the second step, consider the intermediate problem $A 2$ that takes into account the local IC constraints between the skill groups $k-1, k$ and $k+1$. In the solution $\left(c^{A 2}, y^{A 2}\right)$ to problem $A 2$, both downward IC constraints are binding with $\nu_{k-1}^{D}>0$ and $\nu_{k}^{D}>0$. Consequently, we have $y_{k-1}^{A 2}<y_{k-1}^{\alpha R}, y_{k}^{A 2}<y_{k}^{\alpha R}, \delta_{k-1}^{A 2}<\delta_{k-1}^{\alpha R}$ and $\delta_{k+1}^{A}>\delta_{k+1}^{\alpha R}$, while $y_{k-2}^{A 2}=y_{k-2}^{\alpha R}$ and $\delta_{k-2}^{A 2}=\delta_{k-2}^{\alpha R}$. These inequalities imply that $\left(c^{A 2}, y^{A 2}\right)$ violates the downward IC between skill groups $k-2$ and $k-1$ for any $\alpha \in \mathcal{A}^{D}$. The same arguments can be repeated to show that, in the solution to problem $B$ that takes into account the 
local ICs between skill groups $\{1, \ldots, k+1\}$ only, all downward IC constraints are binding and labor supply in each skill group $j \in\{1, \ldots, k\}$ is downwards distorted at the intensive margin.

For the third step, note that the solution to problem $B$ involves $y_{k+1}^{B}=y_{k+1}^{\alpha R}, \delta_{k+1}^{B}>$ $\delta_{k+1}^{\alpha R}$ and $\delta_{k+2}^{B}=\delta_{k+2}^{\alpha R}$. Hence, this allocation violates the upward IC constraint between skill groups $k+1$ and $k+2$ for any $\alpha \in \mathcal{A}^{D}$. In problem $B 2$ that takes into account the local ICs between skill groups $\{1, \ldots, k+2\}$ only, all downwards IC constraints are binding again. Hence, we have $y_{k+1}^{B 2}<y_{k+1}^{\alpha R}, y_{k+2}^{B 2}=y_{k+2}^{\alpha R}, \delta_{k+2}^{B 2}>\delta_{k+2}^{\alpha R}$ and $\delta_{k+3}^{B 3}=\delta_{k+3}^{\alpha R}$, which ensures that the downward IC between skill groups $k+2$ and $k+3$ is violated. The same arguments can be repeated to show that, in allocation $\left(c^{\alpha}, y^{\alpha}\right)$, all downward IC constraints along the skill dimension are binding and that labor supply is downwards distorted at the intensive margin in each skill group $j \in J_{-n}$.

With respect to the distortions at the extensive margin, the previous arguments imply that $\delta_{1}^{\alpha}=y_{1}^{\alpha}-h\left(y_{1}^{\alpha}, \omega_{1}\right)+\left(\alpha_{1}-1\right) / A_{1}\left(\delta_{1}^{\alpha}\right)-\nu_{1}^{D} /\left[f_{1} g_{1}\left(\delta_{1}^{\alpha}\right)\right]$ with $\nu_{1}^{D}>0$. Hence, labor supply in skill group 1 is downwards distorted at the extensive margin if and only if $\alpha_{1}$ is smaller than

$$
\gamma^{D}:=1+\frac{\nu_{1}^{D}}{f_{1} G_{1}\left(\delta_{1}^{\alpha}\right)}+A_{1}\left(\delta_{1}^{\alpha}\right)\left[\delta^{*}\left(\omega_{1}\right)-y_{1}^{\alpha}+h\left(y_{1}^{\alpha}, \omega_{1}\right)\right]
$$

where $\gamma^{D}>1$ because $\nu_{1}^{D}>0$ and because $y_{1}^{\alpha}<y_{1}^{\alpha R}$ implies that $y_{1}^{\alpha}-h\left(y_{1} \alpha, \omega_{1}\right)<\delta^{*}\left(\omega_{1}\right)$. For each higher skill group $j>1$, labor supply is downwards distorted at the extensive margin as well because $\delta_{j}^{\alpha}<y_{j}^{\alpha}-h\left(y_{j}^{\alpha}, \omega_{j}\right) \leq \delta^{*}\left(\omega_{j}\right)$, where the first inequality follows from Lemma 17.

\section{Proof of Proposition 3}

Proof. For $\alpha \in \mathcal{A}^{N},\left(c^{\alpha R}, y^{\alpha R}\right)$ satisfies all local IC constraints along the skill dimension. Hence, the same allocation also solves the non-relaxed problem of optimal taxation, i.e., $\left(c^{\alpha}, y^{\alpha}\right)=\left(c^{\alpha R}, y^{\alpha R}\right)$. By Lemma 3 , labor supply in all skill groups is undistorted at the intensive margin, and upwards (downwards) distorted at the extensive margin if and only if $\alpha_{j}$ is strictly above (strictly below) 1 .

\section{Proof of Proposition 4}

Proof. Fix some $\alpha \in \mathcal{A}^{\chi}$ such that $\alpha_{j+1} \leq \beta_{j}^{U}\left(\alpha_{j}\right)$ for all $j \in\{2, \ldots, n-1\}$, i.e., that the solution to the relaxed problem violates no upward IC except the one between skill groups 1 and 2. This also implies that $\alpha_{1}>\alpha_{2}>1$. The following proof focuses on this case. The results derived below hold for the alternative case that $\left(c^{\alpha R}, y^{\alpha R}\right)$ violates multiple upward ICs a forteriori (a formal proof is available on request). To economize on notation, I write $H_{j}\left(y_{k}\right):=h\left(y_{j}, \omega_{k}\right)-h\left(y_{j}, \omega_{k+1}\right)$ in the following. 
Consider problem $A$ that takes into account the local IC constraints between all pairs of skill groups except $(2,3)$. By Lemma 14 , the upward IC between 1 and 2 is binding with $\nu_{1}^{U}>0$, while all other local ICs are non-binding with $\nu_{j}^{U}=\nu_{j}^{D}=0$ for all $j \in\{3, \ldots, n\}$. By Lemma 15, the solution $\left(c^{A}, y^{A}\right)$ involves $y_{2}^{A}>y_{2}^{\alpha R}, y_{j}^{A}=y_{j}^{\alpha R}$ for all $j \neq 2, \delta_{2}^{A}<\delta_{2}^{\alpha R}$ and $\delta_{j}^{A}=\delta_{j}^{\alpha R}$ for all $j \neq\{1,2\}$. Recall that the relaxed problem's solutions satisfies both neglected IC constraints, i.e.,

$$
H_{2}\left(y_{2}^{\alpha R}\right) \leq \delta_{3}^{\alpha R}-\delta_{2}^{\alpha R} \leq H_{2}\left(y_{3}^{\alpha R}\right)
$$

Note that $y_{2}^{A}>y_{2}^{\alpha R}$ implies that $H_{2}\left(y_{2}^{A}\right)>H_{2}\left(y_{2}^{\alpha R}\right)$ by $h_{y w}<0$. Hence, $\left(c^{A}, y^{A}\right)$ may violate the downward IC between 2 and 3, the corresponding upward IC or none of them. If $\left(c^{A}, y^{A}\right)$ satisfies both IC constraints, we have $\left(c^{\alpha}, y^{\alpha}\right)=\left(c^{A}, y^{A}\right)$. In the following, I consider the remaining two cases.

First, assume that $\left(c^{A}, y^{A}\right)$ violates the downward IC constraint between skill groups 2 and 3 . Consider problem $B$ that takes into account all local IC constraints except those between 3 and 4 . In its solution $\left(c^{B}, y^{B}\right)$, the upward IC between 1 and 2 and the downward IC between 2 and 3 are binding with $\nu_{1}^{U}>0$ and $\nu_{2}^{D}>0$. Hence, we have $\delta_{2}^{B}<\delta_{2}^{\alpha R}$ and $\delta_{3}^{B}>\delta_{3}^{\alpha R}$. By the binding downward IC, we have $H_{2}\left(y_{2}^{B}\right)=\delta_{3}^{B}-\delta_{2}^{B}>$ $\delta_{3}^{\alpha R}-\delta_{2}^{\alpha R} \geq H_{2}\left(y_{2}^{\alpha R}\right)$. This ensures that $y_{2}^{B}>y_{2}^{\alpha R}$ by $h_{y \omega}<0$. Note further that (i) $\delta_{2}^{B}<\delta_{2}^{A}$ and (ii) $y_{j}^{B}=y_{j}^{\alpha R}$ for all $j \in J /\{2\}$ and $\delta_{j}^{B}=\delta_{j}^{\alpha R}$ for all $j \in J /\{1,2,3\}$. By (i), the upward IC between 1 and 2 continues to be binding. By (ii), allocation $\left(c^{B}, y^{B}\right)$ satisfies the (neglected) upward IC between skill groups 3 and 4, and may satisfy or violate the corresponding downward IC. Adding the IC constraints for all skill groups above 3 stepwise, similar arguments as in the proof to Proposition 3 can be applied to show that the optimal allocation $\left(c^{\alpha}, y^{\alpha}\right)$ satisfies the following conditions:

(a) the upward IC between skills 1 and 2 is binding and $y_{2}^{\alpha}>y_{2}^{\alpha R}$,

(b) $y^{\alpha}$ is upwards distorted at the extensive margin in skill groups 1 and 2 by Lemma 16 .

(c) there is a unique skill group $l \in\{3, \ldots, n\}$ such that the skill groups 2 and $l$ are downwards-linked, while all skill groups between $l$ and $n$ are unlinked.

Second, assume that $\left(c^{A}, y^{A}\right)$ violates the upward IC constraint between skill groups 2 and 3. Then, allocation $\left(c^{B}, y^{B}\right)$ involves binding upward ICs between 1 and 2 as well as between 2 and 3 . Hence, we have $\delta_{3}^{B}<\delta_{3}^{\alpha R}$ and $y_{j}^{B}>y_{j}^{\alpha R}$ for $j \in\{2,3\}$. As with allocation $\left(c^{A}, y^{A}\right)$ before, allocation $\left(c^{B}, y^{B}\right)$ may violate the downward IC between 3 and 4 , the corresponding upward IC or none of both. The arguments in the previous paragraph can be applied again to show that both upward ICs continue to be binding in $\left(c^{\alpha}, y^{\alpha}\right)$ and that $y_{j}^{\alpha}>y_{j}^{\alpha R}$ for $j \in\{2,3\}$. Besides, labor supply in skill groups $\{1,2,3\}$ is upwards distorted in $\left(c^{\alpha}, y^{\alpha}\right)$. The same arguments can be repeated to show that there always exists a skill group $k \in\{2, \ldots, n-1\}$ such that the skill groups 1 and $k$ are upwardslinked and (a) either all skill groups between $k$ and $n$ are unlinked or (b) there is a skill 
group $l \in\{k+1, \ldots, n\}$ such that the skill groups $k$ and $l$ are downwards-linked, while all skill groups between $l$ and $n$ are unlinked. Finally, note that skill groups 1 and $n$ cannot be upwards-linked, i.e., $k \neq n$, by Proposition 1 .

\section{Proof of Proposition 5}

Proposition 5 provides conditions that ensure the existence of regular combinations of $\Psi$ and $\gamma$ such that the endogenous (average) welfare weights $\bar{\alpha}$ are elements of the set $\mathcal{A}^{U}$. It is proven by Lemmas 21 and 22 below by example, for $\Psi$ being equal to the identity function, i.e., $\Psi(x)=x$ for all $x \in \mathbb{R}$.

Lemma 21. Consider a sequence $\alpha^{\prime}(\tau)=\left(\alpha_{j}^{\prime}(\tau)\right)_{j=2}^{n}$ such that $\alpha_{2}^{\prime}(\tau)=\tau$ and $\alpha_{j+1}^{\prime}(\tau)=$ $\beta_{j}^{D}\left(\alpha_{j}^{\prime}(\tau)\right)$ for all $j \in\{2, \ldots, n-1\}$. For any $\tau \in(1, \chi)$, there is a unique natural number $m \geq 3$ such that $\alpha_{j}^{\prime}(\tau)<1$ for all $j \in\{m, \ldots, n\}$.

Proof. By Lemma 13, $\beta_{j}^{D}\left(\alpha_{j}\right)<\alpha_{j}$ for all $\alpha_{j} \geq 1$ and $\beta_{j}^{D}\left(\alpha_{j}\right)<1$ for all $\alpha_{j}<1$ (for any $\left.j \in J_{-n}\right)$. It remains to show that there is a number $\mu^{\prime}>0$ such that $\alpha_{j}-\beta_{j}^{D}\left(\alpha_{j}\right)>\mu^{\prime}$ for all $j \in J$ and all $\alpha_{j} \in[1, \chi)$. If this is true, $\alpha^{\prime}(\tau)<\max \left\{\tau-(j-2) \mu^{\prime}, 1\right\}$ for all $j \geq 2$. Hence, $\alpha_{j}^{\prime}(\tau)<1$ if and only if $j>2+\frac{\tau-1}{\mu^{\prime}}$, which directly implies that $\alpha_{n}<1$ whenever $n$ is large enough. The following proof shows that this is true under Condition 3 .

With some abuse of notation, I henceforth denote by $\delta_{k}^{\alpha R}\left(\alpha_{k}\right)$ the level of $\delta_{k}$ in $\left(c^{\alpha R}, y^{\alpha R}\right)$ given social weight $\alpha_{k}$. By construction, function $\beta_{j}^{D}$ satisfies

$$
\delta_{j+1}^{\alpha R}\left(\beta_{j}^{D}(x)\right)-\delta_{j}^{\alpha R}(x)=h\left(y_{j}^{\alpha R}, \omega_{j}\right)-h\left(y_{j}^{\alpha R}, \omega_{j+1}\right),
$$

while, for all $x \geq 1, \delta_{j+1}^{\alpha R}(x)-\delta_{j}^{\alpha R}(x) \geq \delta^{*}\left(\omega_{j+1}\right)-\delta^{*}\left(\omega_{j}\right)$ by Lemma 10 . Consequently, we have

$$
\begin{aligned}
\delta_{j+1}^{\alpha R}(x)-\delta_{j+1}^{\alpha R}\left(\beta_{j}^{D}(x)\right) & \geq \delta^{*}\left(\omega_{j+1}\right)-\delta^{*}\left(\omega_{j}\right)-\left[h\left(y_{j}^{\alpha R}, \omega_{j}\right)-h\left(y_{j}^{\alpha R}, \omega_{j+1}\right)\right] \\
& =y_{j+1}^{\alpha R}-h\left(y_{j+1}^{\alpha R}, \omega_{j+1}\right)-\left[y_{j}^{\alpha R}-h\left(y_{j}^{\alpha R}, \omega_{j+1}\right)\right] \\
& =\int_{y_{j}^{\alpha R}}^{y_{j+1}^{\alpha R}}\left[1-h_{y}\left(y, \omega_{j+1}\right)\right] d y \\
& =\int_{y_{j}^{\alpha R}}^{y_{j+1}^{\alpha R}}\left[1-\frac{1}{1+\frac{h_{y}\left(y_{j+1}^{\alpha R}, \omega_{j+1}\right)-h_{y}\left(y, \omega_{j+1}\right)}{h_{y}\left(y, \omega_{j+1}\right)}}\right] d y
\end{aligned}
$$

where I exploit that $h_{y}\left(y_{j+1}^{\alpha R}, \omega_{j+1}\right)=1$. Let $\hat{y}(\omega):=\arg \max _{y \in \mathbb{R}} y-h(y, \omega)$, and $\hat{w}(y):=$ $(\hat{y})^{-1}(y)$ the corresponding inverse function. Then, the following inequality holds

$$
\begin{aligned}
\frac{h_{y}\left(y_{j+1}^{\alpha R}, \omega_{j+1}\right)-h_{y}\left(y, \omega_{j+1}\right)}{h_{y}\left(y, \omega_{j+1}\right)} & =\int_{\hat{w}(y)}^{\omega_{j+1}} \frac{h_{y y}\left(\hat{y}(\omega), \omega_{j+1}\right)}{h_{y}\left(y, \omega_{j+1}\right)} \frac{d \hat{y}(\omega)}{d \omega} d \omega \\
& =-\int_{\hat{w}(y)}^{\omega_{j+1}} \frac{h_{y y}\left(\hat{y}(\omega), \omega_{j+1}\right)}{h_{y}\left(y, \omega_{j+1}\right)} \frac{h_{y \omega}(\hat{y}(\omega), \omega)}{h_{y y}(\hat{y}(\omega), \omega)} d \omega
\end{aligned}
$$




$$
\begin{aligned}
& >-\int_{\hat{w}(y)}^{\omega_{j+1}} \frac{\hat{y}(\omega) h_{y y}\left(\hat{y}(\omega), \omega_{j+1}\right)}{h_{y}\left(\hat{y}(\omega), \omega_{j+1}\right)} \frac{\omega h_{y \omega}(\hat{y}(\omega), \omega)}{\hat{y}(\omega) h_{y y}(\hat{y}(\omega), \omega)} \frac{1}{\omega} d \omega \\
& \geq \int_{\hat{w}(y)}^{\omega_{j+1}} \frac{\mu_{2}}{\mu_{1}} \frac{1}{\omega} d \omega=\frac{\mu_{2}}{\mu_{1}} \ln \left(\frac{\omega_{j+1}}{\hat{w}(y)}\right),
\end{aligned}
$$

where the last line inequality follows from Condition 3 . Hence, we can derive the following lower bound

$$
\begin{aligned}
\delta_{j+1}^{\alpha R}(x)-\delta_{j+1}^{\alpha R}\left(\beta_{j}^{D}(x)\right) & >\int_{y_{j}^{\alpha R}}^{y_{j+1}^{\alpha R}}\left[1-\frac{1}{1+\frac{\mu_{2}}{\mu_{1}} \ln \left(\frac{\omega_{j+1}}{\hat{w}(y)}\right)}\right] d y=\int_{y_{j}^{\alpha R}}^{y_{j+1}^{\alpha R}} \frac{\ln \left(\frac{\omega_{j+1}}{\hat{w}(y)}\right)}{\frac{\mu_{1}}{\mu_{2}}+\ln \left(\frac{\omega_{j+1}}{\hat{w}(y)}\right)} d y \\
& >\int_{y_{j}^{\alpha R}}^{\hat{y}\left(\omega^{\prime}\right)} \frac{\ln \left(\frac{\omega_{j+1}}{\omega^{\prime}}\right)}{\frac{\mu_{1}}{\mu_{2}}+\ln \left(\frac{\omega_{j+1}}{\omega^{\prime}}\right)} d y=\frac{\ln \left(\frac{\omega_{j+1}}{\omega^{\prime}}\right)}{\frac{\mu_{1}}{\mu_{2}}+\ln \left(\frac{\omega_{j+1}}{\omega^{\prime}}\right)}\left[\hat{y}\left(\omega^{\prime}\right)-y_{j}^{\alpha R}\right] \\
& \geq \frac{\ln \left(\frac{\omega_{j+1}}{\omega^{\prime}}\right)}{\frac{\mu_{1}}{\mu_{2}}+\ln \left(\frac{\omega_{j+1}}{\omega^{\prime}}\right)} \mu_{2} \ln \left(\frac{\omega^{\prime}}{\omega_{j}}\right) y_{j}^{\alpha R}
\end{aligned}
$$

for any $\omega^{\prime} \in\left(\omega_{j}, \omega_{j+1}\right)$. In particular, let $\omega^{\prime}=\sqrt{\omega_{j} \omega_{j+1}}$ and recall that, by assumption, $\omega_{j+1} / \omega_{j} \geq 1+\varepsilon$ for all $j \in J_{-n}$ and some number $\varepsilon>0$. Denoting $\tilde{\varepsilon}=\ln (1+\varepsilon)$, we get

$$
\delta_{j+1}^{\alpha R}(x)-\delta_{j+1}^{\alpha R}\left(\beta_{j}^{D}(x)\right)>\underbrace{\frac{\left(\mu_{2} \tilde{\varepsilon}\right)^{2}}{\mu_{1}+\mu_{2} \tilde{\varepsilon}}}_{:=\mu_{3}} y_{j}^{\alpha R},
$$

where $\mu_{3}$ is bound away from zero for any $j \in J_{-n}$.

From equation (33), the left-hand side of the last inequality can also be written as

$$
\int_{\beta_{j}^{D}(x)}^{x} \frac{d \delta_{j+1}^{\alpha R}\left(x^{\prime}\right)}{d \alpha} d x^{\prime}=\int_{\beta_{j}^{D}(x)}^{x} \frac{\left[A_{j+1}\left(\delta_{j}^{\alpha R}\left(x^{\prime}\right)\right)\right]^{-1}}{1+\left(x^{\prime}-1\right)\left[1-\frac{a_{j}\left(\delta_{j}^{\alpha R}\left(x^{\prime}\right)\right)}{A_{j}\left(\delta_{j}^{\alpha R}\left(x^{\prime}\right)\right)}\right]} d x^{\prime}
$$

By Lemma 2, $A_{j}\left(\delta_{j}^{\alpha R}\right)>A_{n}\left(\delta_{n}^{\alpha R}\right)$ in any allocation that satisfies all downward IC constraints. Moreover, $\alpha_{j}^{\prime}(\tau)<\alpha_{2}=\tau$ for all $j>2$, and $1-a_{j}\left(\delta_{j}^{\alpha R}\right) / A_{j}\left(\delta_{j}^{\alpha R}\right)<1 /(\chi-1)$ for any $\alpha \in \mathcal{A}^{\chi}$ by the construction of $\chi$ (see Lemma 3). Hence, $d \delta_{j}^{\alpha R}\left(x^{\prime}\right) / d \alpha$ is strictly smaller than $\mu_{4}:=A_{n}\left[\delta_{n}^{\alpha R}(\tau)\right]^{-1}(\chi-1) /(\chi-\tau)$ for all $j \in J$ and $x^{\prime} \in\left[1, \alpha_{2}\right]$. For all $j \in J \backslash\{1\}$ and $\alpha \in[1, \tau]$, we consequently have

$$
\begin{aligned}
& \int_{\beta_{j}^{D}(x)}^{x} \mu_{4} d x^{\prime}=\mu_{4}\left[x-\beta_{j}^{D}(x)\right]>\mu_{3} y_{j}^{\alpha R} \\
\Leftrightarrow & x-\beta_{j}^{D}(x)>\frac{\mu_{3}}{\mu_{4}} y_{2}^{\alpha R}=: \mu^{\prime} .
\end{aligned}
$$

As argued above, this ensures that $\tau-\alpha_{j}^{\prime}(\tau)=\sum_{k=2}^{j-1}\left[\alpha_{k}^{\prime}(\tau)-\beta_{k}^{D}\left(\alpha_{k}^{\prime}(\tau)\right)\right]>(j-2) \mu^{\prime}$ if $\alpha^{\prime}(\tau) \geq 1$, and $\alpha_{j}^{\prime}(\tau)<1$ for all $j>2+\frac{\tau-1}{\mu^{\prime}}$. Hence, there exists a level $m \in$ $\left[3,2+(\tau-1) / \mu^{\prime}\right)$ such that $\alpha_{j}<1$ if and only if $j \geq m$. 
Lemma 22. Let Condition 3 and $\frac{\omega_{2}}{\omega_{1}}<a_{1}^{u}(\zeta)$ be satisfied for some $\zeta \in(1, \chi)$. Then, there exist a number $m \geq 3$ and two vectors $\left(\phi_{j}\right)_{j=1}^{n},\left(\tilde{\delta}_{j}\right)_{j=1}^{n}$ with $\phi_{j+1} \geq \phi_{j}, \phi_{m}>1>\phi_{1}=0$ and $\delta_{j}^{\prime} \in(\underline{\delta}, \bar{\delta})$ for all $j \in J$ such that, if

(i) $n \geq m$ and

(ii) $\sum_{j=1}^{n} f_{j} G_{j}\left(\delta_{j}^{\prime}\right) \phi_{j}>1$,

there exist regular combinations of $\Psi$ and $\gamma$ for which $\alpha \in \mathcal{A}^{U}$.

Proof. Assume that $\Psi$ is the identity function and fix some number $\hat{\delta} \in\left(\delta^{*}\left(\omega_{n}\right), \bar{\delta}\right)$. Consider the weighting function $\gamma^{\prime}$ such that

$$
\gamma^{\prime}\left(\omega_{j}, \delta\right)=\left\{\begin{array}{ccc}
\gamma_{j}^{\prime}=\bar{\beta}_{1} & \text { for } & j \in\{1,2\}, \delta<\hat{\delta}, \\
\left.\gamma_{j}^{\prime}=\max \left\{\beta_{j-1}^{D}\left(\gamma_{j-1}\right), \underline{\beta}_{j}, \ldots, \underline{\beta}_{n-1}\right\}\right\} & \text { for } & j \in\{3, \ldots, n\}, \delta<\hat{\delta}, \\
\gamma_{0}^{\prime}=\frac{1-\sum_{j=1}^{n} f_{j} G_{j}(\hat{\delta}) \gamma_{j}}{1-\sum_{j=1}^{n} f_{j} G_{j}(\hat{\delta})} & \text { for } & j \in J, \delta \geq \hat{\delta},
\end{array}\right.
$$

where $\bar{\beta}_{1}$ is a fixed point of function $\beta_{1}^{U}$. By Lemma $12, \bar{\beta}_{1}$ exists, is unique and is located in $(1, \chi)$ if $\omega_{2} / \omega_{1}<a^{U}(\zeta)$ for some $\zeta \in(1, \chi)$ under Conditions 1 and 2 .

By Lemma 21, Condition 3 ensures that there is a unique number $m \geq 3$ such that $\gamma_{j}^{\prime}<1$ for all $j \in[m, n]$ if $n \geq m$. Assume that the latter condition is met (i.e., (ii) in the Lemma above). Let $\delta_{j}^{\prime}$ be equal to the level of $\delta_{j}$ in the relaxed problem's solution $\left(c^{\alpha R}, y^{\alpha R}\right)$ given $\alpha_{j}=\gamma_{j}^{\prime}$ for each $j \in J$. Then, the average weight $\bar{\alpha}_{n}$ (among workers in skill group $n$ ) equals $\gamma_{n}^{\prime}<1$, and $\delta_{n}^{\prime}<\delta^{*}\left(\omega_{n}\right)<\hat{\delta}$. Moreover, $\bar{\alpha}_{j}=\gamma_{j}^{\prime}$ and $\delta_{j}^{\prime}<\delta_{n}^{\prime}<\hat{\delta}$ for all $j \in J_{-n}$ (as all downward IC constraints are satisfied, see proof of Lemma 2p. Finally, the average social weight of the unemployed is given by $\bar{\alpha}_{0}=\left[1-\sum_{j=1}^{n} f_{j} G_{j}\left(\delta_{j}^{\prime}\right) \gamma_{j}^{\prime}\right] /\left[1-\sum_{j=1}^{n} f_{j} G_{j}\left(\delta_{j}^{\prime}\right)\right]$.

By construction, $\bar{\alpha}$ satisfies $\bar{\alpha}_{2} \geq \beta_{1}^{U}\left(\bar{\alpha}_{1}\right)$ and $\bar{\alpha}_{j+1} \geq \beta_{j}^{D}\left(\bar{\alpha}_{j}\right)$ for all $j \in\{2, \ldots, n-1\}$. Moreover, $\bar{\alpha}_{j} \geq \bar{\alpha}_{j+1}$ for all $j \in J$ and $\bar{\alpha}_{j}>\bar{\alpha}_{j+1}$ for all $j \in\{2, \ldots, m\}$. We also have $\bar{\alpha}_{0}>\bar{\alpha}_{1}=\bar{\beta}_{1}$ if and only if

$$
\sum_{j=1}^{n} f_{j} G_{j}\left(\delta_{j}^{\prime}\right)\left[\bar{\beta}_{1}-\gamma_{j}^{\prime}\right]>\bar{\beta}_{1}-1
$$

Let $\phi_{j}=\left(\bar{\beta}_{1}-\gamma_{j}^{\prime}\right) /\left(\bar{\beta}_{1}-1\right)$ for all $j \in J$. Then, $\bar{\alpha}_{0}>\bar{\beta}_{1}$ if conditions (ii) and (ii) in Lemma 22 are satisfied.

For the final step, note that the average welfare weights $\bar{\alpha}$ are not an element of $\mathcal{A}^{U}$ given $\gamma^{\prime}$ because $\bar{\alpha}_{1}=\bar{\alpha}_{2}=\bar{\beta}_{1}$. Consider the weighting function $\gamma^{\varepsilon}$ such that

$$
\gamma^{\varepsilon}\left(\omega_{j}, \delta\right)=\left\{\begin{array}{ccc}
\gamma_{1}^{\varepsilon}=\bar{\beta}_{1}+\varepsilon_{1}+\varepsilon_{2} & \text { for } & j=1, \delta<\hat{\delta}, \\
\gamma_{2}^{\varepsilon}=\bar{\beta}_{1}+\varepsilon_{2} & \text { for } & j=2, \delta<\hat{\delta}, \\
\gamma_{j}^{\varepsilon}=\max \left\{\beta_{j-1}^{D}\left(\gamma_{j-1}^{\varepsilon}\right), \underline{\beta}_{j}, \ldots, \underline{\beta}_{n-1}\right\} & \text { for } & j \in\{3, \ldots, n\}, \delta<\hat{\delta}, \\
\gamma_{0}^{\varepsilon}=\frac{1-\sum_{j=1}^{n} f_{j} G_{j}\left(\delta_{j}^{\varepsilon}\right) \gamma_{j}^{\varepsilon}}{1-\sum_{j=1}^{n} f_{j} G_{j}\left(\delta_{j}^{\varepsilon}\right)} & \text { for } & j \in J, \delta \geq \hat{\delta},
\end{array}\right.
$$


where $\delta_{j}^{\varepsilon}$ is the level of $\delta_{j}$ in the relaxed problem's solution given $\alpha_{j}=\gamma_{j}^{\varepsilon}$ for each $j \in J$. Fix $\varepsilon_{2}$ at some level in $\left(0, \chi-\bar{\beta}_{1}\right)$. By Lemma 12 there is a unique number $\bar{\varepsilon}>0$ such that $\gamma_{2}^{\varepsilon} \geq \beta_{1}^{U}\left(\gamma_{1}^{\varepsilon}\right)$ for each $\varepsilon_{1} \in(0, \bar{\varepsilon})$. If $\varepsilon_{1}>0$ and $\varepsilon_{2}>0$ are sufficiently small, the resulting average weight $\bar{\alpha}_{0}$ continues to be strictly larger than $\gamma_{1}^{\varepsilon}$. Hence, $\bar{\alpha}_{j}>\bar{\alpha}_{j+1}$ for all $j \in\{0, \ldots, m-1\}$. Consequently, $\bar{\alpha} \in \mathcal{A}^{U}$ results if $\gamma_{j+1}^{\varepsilon}<\gamma_{j}^{\varepsilon}$ for all $j \in\{m, \ldots, n-1\}$ as well. (If $\gamma^{\varepsilon}$ involves $\gamma_{j+1}^{\varepsilon}=\gamma_{j}^{\varepsilon}$ for some $j \geq m$, then one can construct a weighting function $\gamma$ that is strictly decreasing over the skill dimension and has otherwise identical properties, i.e., for which $\bar{\alpha} \in \mathcal{A}^{U}$.)

\section{Proof of Lemma 6}

Proof. First, note that minimizing the deadweight loss (6) is equivalent to maximizing the term $\sum_{j=1}^{n} f_{j} \int_{\underline{\delta}}^{\delta_{j}} g_{j}(\delta)\left[y_{j}-h\left(y_{j}, \omega_{j}\right)-\delta\right] d \delta$ over $c$ and $y$. The Lagrangian for the problem of efficient redistribution is hence given by

$$
\begin{aligned}
\mathcal{L}= & \sum_{j=1}^{n} f_{j} \int_{\underline{\delta}}^{\delta_{j}} g_{j}(\delta)\left[y_{j}-h\left(y_{j}, \omega_{j}\right)-\delta\right] d \delta+\lambda_{F}\left[\sum_{j=3}^{n} f_{j} G_{j}\left(\delta_{j}\right)\left(y_{j}-c_{j}\right)-R\right] \\
& +\lambda_{E}\left[\sum_{j=1}^{2} f_{j} G_{j}\left(\delta_{j}\right)\left(c_{j}-y_{j}\right)+\sum_{j=1}^{n} f_{j}\left[1-G_{j}\left(\delta_{j}\right)\right] c_{0}-R\right] \\
& +\mu\left[c_{1}-h\left(y_{1}, \omega_{1}\right)-c_{2}+h\left(y_{2}, \omega_{1}\right)\right],
\end{aligned}
$$

where I have included the constraints that resources $R$ are transferred away from the workers in skill groups 3 and higher (Lagrange parameter $\lambda_{F}$ ), the same resources are transferred towards the unemployed and the workers in skill groups 1 and $2\left(\lambda_{E}\right)$, and the upward IC constraint between the workers in skill groups 1 and $2(\mu)$. Note that the first two constraints are binding in the solution of the efficient redistribution problem for any $R>0$. Hence, $\lambda_{E}$ and $\lambda_{F}$ are strictly positive in any solution.

First, I solve the problem ignoring the upward IC constraint, and denote the solution by $\left(c^{E R}, y^{E R}\right)$. The FOCs with respect to $c_{j}$ and $y_{j}$ for $j \in\{1,2\}$ are given by

$$
\begin{aligned}
\mathcal{L}_{c_{j}}= & \left(1-\lambda_{E}\right) f_{j} g_{j}\left(\delta_{j}^{E R}\right)\left[y_{j}^{E R}-h\left(y_{j}^{E R}, \omega_{j}\right)-\delta_{j}^{E R}\right]+\lambda_{E} f_{j} G_{j}\left(\delta_{j}^{E R}\right) \stackrel{!}{=} 0, \text { and } \\
\mathcal{L}_{y_{j}}= & f_{j} G_{j}\left(\delta_{j}^{E R}\right)\left[1-h_{y}\left(y_{j}^{E R}, \omega_{j}\right)\right] \\
& -h_{y}\left(y_{j}^{E R}, \omega_{j}\right)\left(1-\lambda_{E}\right) f_{j} g_{j}\left(\delta_{j}^{E R}\right)\left[y_{j}^{E R}-h\left(y_{j}^{E R}, \omega_{j}\right)-\delta_{j}^{E R}\right]-\lambda_{E} f_{j} G_{j}\left(\delta_{j}^{E R}\right) \stackrel{!}{=} 0 .
\end{aligned}
$$

The combination of both FOCs yields that labor supply in both low-skill groups is undistorted at the intensive margin, $h_{y}\left(y_{j}^{E R}, \omega_{j}\right)=1$, which also implies $y_{j}^{E R}-h\left(y_{j}^{E R}, \omega_{j}\right)=$ $\delta^{*}\left(\omega_{j}\right)$. Rearranging the first condition, we additionally get the inverse elasticity rule 23],

$$
\delta_{j}^{E R}-\delta^{*}\left(\omega_{j}\right)=c_{j}^{E R}-y_{j}^{E R}-c_{0}^{E R}=\frac{\lambda^{E}}{1-\lambda^{E}} \frac{G_{j}\left(\delta_{j}^{E R}\right)}{g_{j}\left(\delta_{j}^{E R}\right)} .
$$


From the second-order condition, $\lambda_{E}$ has to attain a value below some bound $\hat{\lambda}<1$ at any solution (the exact level of $\hat{\lambda}$ depends on the type distribution $K)$. Hence, $\delta_{j}^{E R}>\delta^{*}\left(\omega_{j}\right)$ for $j \in\{1,2\}$ and any $R>0$ such that an interior solution exists. From Lemma 2 , we know that $\eta_{1}(c, y)=\frac{g_{1}\left(\delta_{1}\right)}{G_{1}\left(\delta_{1}\right)}>\eta_{2}(c, y)=\frac{g_{2}\left(\delta_{2}\right)}{G_{2}\left(\delta_{2}\right)}$ in any incentive-compatible allocation, which implies that $c_{2}^{E R}-y_{2}^{E R}>c_{1}^{E R}-y_{1}^{E R}$. Hence, allocation $\left(c^{E R}, y^{E R}\right)$ satisfies the downward IC (21), and might satisfy or violate the upward IC (22).

Fix a number $R>0$, the type distribution $K$ and all skill levels except $\omega_{2}$. Similar arguments as in the proof of Lemma 12 (ii) can be applied to show that there is some number $a^{E}>1$ such that the upward IC constraint is violated by the allocation $\left(c^{E R}, y^{E R}\right)$ whenever $\omega_{2} \in\left(\omega_{1}, a^{E} \omega_{1}\right)$. First, consider the limit case $\omega_{2}=\omega_{1}$. In this case, $\lambda_{E} \in(0,1)$ and $\delta_{2}^{E R} \geq \delta_{1}^{E R}$ by Condition 1. As shown in the proof of Lemma 12 (ii), allocation $\left(c^{E}, y^{E}\right)$ unambiguously violates the upward IC constraint after a marginal increase in $\omega_{2}$ for any fixed $\lambda_{E} \in(0,1)$. Because $\omega_{2}$ and all other variables enter the maximization program continuously, $\omega_{2}$ also affects the value of the Lagrange parameter $\lambda_{E}$ continuously. Hence, $\lambda_{E}$ is bounded away from 0 and 1 after a marginal increase in $\omega_{2}$. Thus, there is a number $a^{E}>1$ such that the upward IC constraint is violated for any $\omega_{2} \in\left(\omega_{1}, a^{E} \omega_{1}\right)$.

Next, consider a combination of $R>0$ and $\omega_{2} \in\left(\omega_{1}, \omega_{3}\right)$ such that $\left(c^{E R}, y^{E R}\right)$ violates the upward IC constraint. Taking into account this constraint for the Lagrangian, the adjusted FOCs with respect to $c_{1}, c_{2}$ and $y_{1}$ are given by

$$
\begin{aligned}
\mathcal{L}_{c_{1}}= & \left(1-\lambda_{E}\right) f_{1} g_{1}\left(\delta_{1}^{E}\right)\left[y_{1}^{E}-h\left(y_{1}^{E}, \omega_{1}\right)-\delta_{1}^{E}\right]+\lambda_{E} f_{1} G_{1}\left(\delta_{1}^{E}\right)+\mu \stackrel{!}{=} 0, \\
\mathcal{L}_{c_{2}}= & \left(1-\lambda_{E}\right) f_{2} g_{2}\left(\delta_{2}^{E}\right)\left[y_{2}^{E}-h\left(y_{2}^{E}, \omega_{2}\right)-\delta_{2}^{E}\right]+\lambda_{E} f_{2} G_{2}\left(\delta_{2}^{E}\right)-\mu \stackrel{!}{=} 0, \text { and } \\
\mathcal{L}_{y_{2}}= & f_{2} G_{2}\left(\delta_{2}^{E}\right)\left[1-h_{y}\left(y_{2}^{E}, \omega_{2}\right)\right]-h_{y}\left(y_{2}^{E}, \omega_{2}\right)\left(1-\lambda_{E}\right) f_{2} g_{2}\left(\delta_{2}^{E}\right)\left[y_{2}^{E}-h\left(y_{2}^{E}, \omega_{2}\right)-\delta_{2}^{E}\right] \\
& -\lambda_{E} f_{2} G_{2}\left(\delta_{2}^{E}\right)+\mu h_{y}\left(2, \omega_{1}\right) \stackrel{!}{=} 0 .
\end{aligned}
$$

Combining the two latter conditions shows that, as usual, $y_{2}^{E}$ is upwards distorted if and only if the upward IC constraint is binding with $\mu>0$,

$$
f_{2} G_{2}\left(\delta_{2}^{E}\right)\left[h_{y}\left(y_{2}^{E}, \omega_{2}\right)-1\right]\left(1-\lambda_{E}\right)=\mu\left[h_{y}\left(y_{2}, \omega_{1}\right)-h_{y}\left(y_{2}, \omega_{2}\right)\right]>0
$$

Using the FOC with respect to $c_{2}$, we can replace $\mu$ in the previous condition to get

$$
\frac{f_{2} G_{2}\left(\delta_{2}^{E}\right)\left[h_{y}\left(y_{2}^{E}, \omega_{2}\right)-1\right]}{h_{y}\left(y_{2}, \omega_{1}\right)-h_{y}\left(y_{2}, \omega_{2}\right)}=\frac{\lambda_{E}}{1-\lambda_{E}} f_{2} G_{2}\left(\delta_{2}^{E}\right)-f_{2} g_{2}\left(\delta_{2}^{E}\right)\left[\delta_{2}^{E}-y_{2}^{E}+h\left(y_{2}^{E}, \omega_{2}\right)\right] \text {. }
$$

Combining the FOCs with respect to $c_{1}$ and $c_{2}$ and inserting $y_{1}^{E}-h\left(y_{1}^{E}, \omega_{1}\right)=\delta^{*}\left(\omega_{1}\right)$ gives

$$
\frac{\lambda_{E}}{1-\lambda_{E}}=\frac{f_{1} g_{1}\left(\delta_{1}^{E}\right)\left[\delta_{1}^{E}-\delta^{*}\left(\omega_{1}\right)\right]+f_{2} g_{2}\left(\delta_{2}^{E}\right)\left[\delta_{2}^{E}-y_{2}^{E}+h\left(y_{2}^{E}, \omega_{2}\right)\right]}{f_{1} G_{1}\left(\delta_{1}^{E}\right)+f_{2} G_{2}\left(\delta_{2}^{E}\right)} .
$$

Inserting this term into the previous function to eliminate the Lagrangian parameter $\lambda^{E}$ and rearranging terms gives the optimality condition (24), which implies that $y_{2}^{E}$ has to be 
chosen so to equate the marginal deadweight losses from distortions at both margins.

\section{B Supplementary material}

\section{B.1 Illustration of labor supply distortions}

In Subsection 3.2. I formally define labor supply distortions at the intensive margin and at the extensive margin. The following Figures 2 and 3 illustrate these definitions graphically. In each figure, point $A$ marks the initial bundle $\left(c^{i}, y^{i}\right)$ allocated to agent $i$. The sets of hypothetical deviations are given by the solid lines through $A$ and $B$. The indifference curves of Agent $i$ are given by the union of the dashed line and point $Z$ (in figure 2 ) and the union of the dashed line and point $A$ (in figure 3 ), respectively, corresponding to the discontinuity in $i$ 's utility due to the fixed $\operatorname{cost} \delta^{i}$.

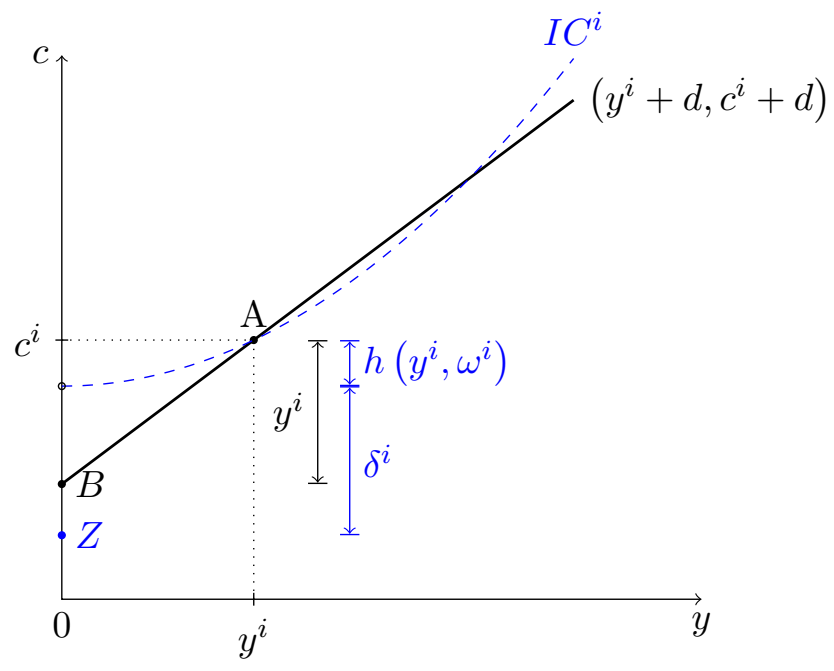

Figure 2: Labor supply distortions, Example 1.

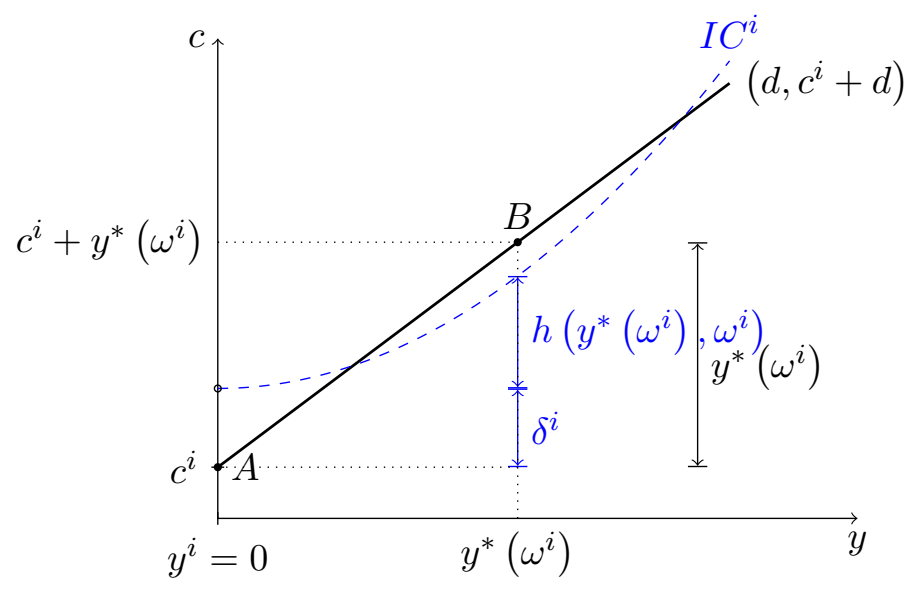

Figure 3: Labor supply distortions, Example 2. 
In Figure 2, $i$ 's output is strictly positive, $y^{i}>0$. In point $A$, the slope of the indifference curve is below 1 , the marginal rate of substitution. Hence, $i$ 's utility could be increased by moving slightly upwards the solid line. Alternatively, $i$ 's utility could also be increased by jumping downwards to point $B$, where output provision is zero. Hence, $i$ 's labor supply is both downwards distorted at the intensive margin and upwards distorted at the extensive margin 51

In Figure 3, $i$ does not provide output, $y^{i}=0$. Jumping upwards to point $B$ with positive output $y^{*}\left(\omega^{i}\right)=\arg \max _{y>0}\left\{y-h\left(y, \omega^{i}\right)\right\}$ would increase $i$ 's utility, as $B$ is located above the indifference curve. Hence, $i$ 's labor supply is downwards distorted at the extensive margin.

\section{B.2 Construction, decomposition and illustration of dead- weight loss}

In the model studied above, if labor supply by all agents is undistorted, the difference between the consumption possibilities that result from providing labor and the total costs of providing labor (measured in consumption units) is maximized. Following the literature, I define the deadweight loss (or excess burden) in any allocation $(c, y)$ as the loss in this difference relative to its maximum. Formally, the deadweight loss in an implementable allocation $(c, y)$ is hence given by

$$
\begin{aligned}
D W L(c, y)= & \sum_{j=1}^{n} f_{j} \int_{\underline{\delta}}^{\delta^{*}\left(\omega_{j}\right)} g_{j}(\delta)\left[\delta^{*}\left(\omega_{j}\right)-\delta\right] d \delta \\
& -\sum_{j=1}^{n} f_{j} \int_{\underline{\delta}}^{\delta_{j}} g_{j}(\delta)\left[y_{j}-h\left(y_{j}, \omega_{j}\right)-\delta\right] d \delta .
\end{aligned}
$$

As usual, the deadweight loss is minimized and equal to zero if labor supply in all skill groups is undistorted at both margins, i.e., if $h_{y}\left(y_{j}, \omega_{j}\right)=1$ and $\delta_{j}=\delta^{*}\left(\omega_{j}\right)=\max _{y>0} y-$ $h\left(y, \omega_{j}\right)$ for every $j \in J$.

Note also that the deadweight loss can be decomposed as follows,

$$
\begin{aligned}
D W L(c, y)= & \sum_{j=1}^{n} f_{j} G_{j}\left(\delta_{j}\right)\left[\delta^{*}\left(\omega_{j}\right)-y_{j}+h\left(y_{j}, \omega_{j}\right)\right] \\
& +\sum_{j=1}^{n} f_{j} \int_{\delta_{j}}^{\delta^{*}\left(\omega_{j}\right)} g_{j}(\delta)\left[\delta^{*}\left(\omega_{j}\right)-\delta\right] d \delta
\end{aligned}
$$

where the first term captures the deadweight loss from distortions at the intensive margin and the second term captures the deadweight loss from distortions at the extensive margin (across all skill groups).

\footnotetext{
${ }^{51}$ In a model without fixed costs as in, e.g., Mirrlees (1971), this would be impossible by construction.
} 
Figure 4 below illustrates the deadweight loss from distortions in skill group $j$ and its decomposition graphically. It depicts the quantity $G_{j}\left(\delta_{j}\right)$ of labor of skill type $\omega_{j}$, and the labor supply $S_{j}$ and labor demand $D_{j}$ (measured in mass of workers) for an allocation that involves bundle $\left(y_{j}, \delta_{j}\right) . D_{j}^{*}$ depicts the labor demand that would result without distortions at the intensive margin, i.e., if each worker would provide efficient output. In particular, the figure depicts a case where labor supply in skill group $j$ is downwards distorted at both margins, $h_{y}\left(y_{j}, \omega_{j}\right)<1$ and $\delta_{j}<y_{j}-h\left(y_{j}, \omega_{j}\right)<\delta^{*}\left(\omega_{j}\right)$. The red shaded area $\left(L_{i}\right)$ depicts the efficiency loss due to intensive-margin distortions, the blue-shaded area $\left(L_{e}\right)$ depicts the efficiency loss due to extensive-margin distortions in this skill group.

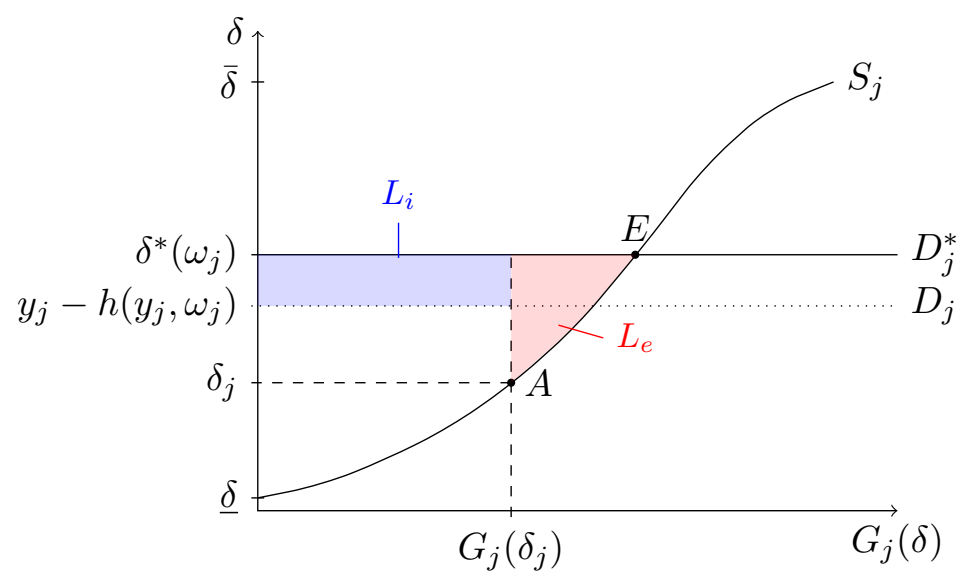

Figure 4: Illustration of deadweight loss in skill group $j$.

\section{B.3 Monotonicity of social weights}

In Subsection 3.4, the endogenous marginal social weights are defined in equations 10 . and (11). In the following, I assume that all type-specifid weight are equal, i.e., that $\gamma(\omega, \delta)=1$ for all $(\omega, \delta) \in \Omega \times \Delta$. Then, concavity of $\Psi$ ensures that $\bar{\alpha}_{0}>\bar{\alpha}_{j}$ for all $j \in J$. For $j \geq 1$, however, the endogenous weight sequence $\bar{\alpha}$ is only ensured to be decreasing if additional conditions on the joint type distribution $K$ are met.

Lemma 23. If $\Psi$ is strictly concave and $\gamma$ is constant over $\Omega \times \Delta, G_{j}$ dominates $G_{j+1}$ in the sense of first-order stochastic dominance for all $j \in J_{-n}$ and Condition 1 holds, $\bar{\alpha}_{j}>\bar{\alpha}_{j+1}$ for all $j \in J_{-n}$ in all implementable allocations.

Proof. Using Lemma 1 and $c_{j}-h\left(y_{j}, \omega_{j}\right)=\delta_{j}+c_{0}$, weight $\bar{\alpha}_{j}$ can be rewritten as a function of $\delta_{j}$,

$$
\bar{\alpha}_{j}\left(\delta_{j}\right)=\frac{1}{z G_{j}\left(\delta_{j}\right)} \int_{\underline{\delta}}^{\delta_{j}} g_{j}(\delta) \Psi^{\prime}\left[\delta_{j}+c_{0}-\delta\right] d \delta,
$$

where $z$ is a normalization parameter. In the following, I show that, in every implementable 
allocation,

$$
z\left[\bar{\alpha}_{j}\left(\delta_{j}\right)-\bar{\alpha}_{j+1}\left(\delta_{j+1}\right)\right]=z\left[\bar{\alpha}_{j}\left(\delta_{j}\right)-\bar{\alpha}_{j+1}\left(\delta_{j}\right)\right]+z\left[\bar{\alpha}_{j+1}\left(\delta_{j}\right)-\bar{\alpha}_{j+1}\left(\delta_{j+1}\right]<0 .\right.
$$

For the term in the first bracket, note that

$$
\begin{aligned}
& z\left[\bar{\alpha}_{j}\left(\delta_{j}\right)-\bar{\alpha}_{j+1}\left(\delta_{j}\right)\right]=\left[\frac{1}{G_{j}\left(\delta_{j}\right)}-\frac{1}{G_{j+1}\left(\delta_{j}\right)}\right] \int_{\underline{\delta}}^{\delta_{j}} \Psi^{\prime}\left[\delta_{j}+c_{0}-\delta\right] d G_{j}(\delta)+ \\
& \frac{1}{G_{j+1}\left(\delta_{j}\right)}\left[\int_{\underline{\delta}}^{\delta_{j}} \Psi^{\prime}\left[\delta_{j}+c_{0}-\delta\right] d G_{j}(\delta)-\int_{\underline{\delta}}^{\delta_{j}} \Psi^{\prime}\left[\delta_{j}+c_{0}-\delta\right] d G_{j+1}(\delta)\right],
\end{aligned}
$$

where the first term is weakly positive by the assumed first-order stochastic dominance. Applying integration by parts, the term in the second bracket can be written as

$$
\left[A_{j}\left(\delta_{j}\right)-A_{j+1}\left(\delta_{j}\right)\right] \Psi^{\prime}\left(c_{0}\right)+\int_{\underline{\delta}}^{\delta_{j}}\left[G_{j}(\delta)-G_{j+1}(\delta)\right] \Psi^{\prime \prime}\left[\delta_{j}+c_{0}-\delta\right] d \delta \geq 0,
$$

where the first term is weakly positive by Condition 1 (ii), and the second term is weakly positive by the assumed first-order stochastic dominance. Hence, the term $\bar{\alpha}_{j}\left(\delta_{j}\right)-\bar{\alpha}_{j+1}\left(\delta_{j}\right)$ is weakly positive.

For the term in the second bracket, note that

$$
\begin{aligned}
\frac{d \bar{\alpha}_{j}}{d \delta_{j}}= & A_{j}\left(\delta_{j}\right) \Psi^{\prime}\left(c_{0}\right)+\frac{1}{G_{j}\left(\delta_{j}\right)} \int_{\underline{\delta}}^{\delta_{j}} g_{j}(\delta) \Psi^{\prime \prime}\left[\delta_{j}+c_{0}-\delta\right] d \delta \\
& -\frac{A_{j}\left(\delta_{j}\right)}{G_{j}\left(\delta_{j}\right)} \int_{\underline{\delta}}^{\delta_{j}} g_{j}(\delta) \Psi^{\prime}\left[\delta_{j}+c_{0}-\delta\right] d \delta
\end{aligned}
$$

Applying integration by parts again, the last term can be rewritten to get

$$
\begin{aligned}
\frac{d \bar{\alpha}_{j}}{d \delta_{j}} & =\frac{1}{G_{j}\left(\delta_{j}\right)} \int_{\underline{\delta}}^{\delta_{j}} g_{j}(\delta) \Psi^{\prime \prime}\left[\delta_{j}+c_{0}-\delta\right] d \delta-\frac{A_{j}\left(\delta_{j}\right)}{G_{j}\left(\delta_{j}\right)} \int_{\underline{\delta}}^{\delta_{j}} G_{j}(\delta) \Psi^{\prime \prime}\left[\delta_{j}+c_{0}-\delta\right] d \delta \\
& =\frac{1}{G_{j}\left(\delta_{j}\right)} \int_{\underline{\delta}}^{\delta_{j}} G_{j}(\delta)\left[A_{j}(\delta)-A_{j}\left(\delta_{j}\right)\right] \Psi^{\prime \prime}\left[\delta_{j}+c_{0}-\delta\right] d \delta<0,
\end{aligned}
$$

where the negative sign follows because $\Psi$ is concave and $A_{j}(\delta)>A_{j}\left(\delta_{j}\right)$ for all $\delta \in\left[\underline{\delta}, \delta_{j}\right)$ due to the log-concavity imposed by Condition 1 (i).

In the following, I provide a simple example to demonstrate that the concavity of $\Psi$ per se does not guarantee decreasing social weights.

Example 1. Assume that $n>2, \omega_{1}=1, \omega_{2}=3 / 2, \underline{\delta}=0, \bar{\delta}=10, h(y, \omega)=\frac{1}{2}\left(\frac{y}{\omega}\right)^{2}$, $\Psi(x)=x^{1 / 2}, \gamma(\omega, \delta)=1$ for all $(\omega, \delta) \in \Omega \times \Delta, g_{1}(\delta)=0.1$ for all $\delta \in \Delta, g_{2}(\delta)=\varepsilon$ for $\delta \in[0,1]$ and $g_{2}(\delta)=\frac{1-\varepsilon}{9}$ for $\delta \in(1,10]$.

Note that function $\Psi$ is strictly concave. Fixed costs types are uniformly distributed in skill group 1, and piecewise uniformly distributed in skill group 2. For $\varepsilon$ below (above) 
$0.1, G_{2}$ dominates (is dominated by) $G_{1}$ in the sense of first-order stochastic dominance.

Consider the allocation $\left(c^{\prime}, y^{\prime}\right)$ with $c_{0}^{\prime}=0.1,\left(c_{1}^{\prime}, y_{1}^{\prime}\right)=(1.1,1),\left(c_{2}^{\prime}, y_{2}^{\prime}\right)=(9 / 4+$ $.1,9 / 4), \delta_{1}^{\prime}=1 / 2$ and $\delta_{2}^{\prime}=9 / 4$. Note that this allocation satisfies both IC constraints between workers with skill types $\omega_{1}$ and $\omega_{2}$ with strict inequalities. The social weights in this allocation are given by

$$
\begin{aligned}
\bar{\alpha}_{0}\left(c_{0}^{\prime}\right) & =\frac{1}{z} \Psi^{\prime}\left(c_{0}^{\prime}\right) \approx \frac{1.581}{z}, \\
\bar{\alpha}_{1}\left(\delta_{1}^{\prime}, c_{0}^{\prime}\right) & =\frac{2}{z}\left[\sqrt{\delta_{1}^{\prime}+c_{0}^{\prime}}-\sqrt{c_{0}^{\prime}}\right] \approx \frac{.917}{z}, \text { and } \\
\bar{\alpha}_{2}\left(\delta_{2}^{\prime}, c_{0}^{\prime}\right) & =\frac{1}{G_{2}\left(\delta_{2}^{\prime}\right) z}\left[\varepsilon\left(\sqrt{\delta_{2}^{\prime}+c_{0}^{\prime}}-\sqrt{\delta_{2}^{\prime}+c_{0}^{\prime}-1}\right)+\frac{1-\varepsilon}{9}\left(\sqrt{\delta_{2}^{\prime}+c_{0}^{\prime}-1}-\sqrt{c_{0}^{\prime}}\right)\right] \\
& \approx \frac{1.265+44.272 \varepsilon}{(1+71 \varepsilon) z},
\end{aligned}
$$

where $z>0$ is again a normalizing parameter. The following result is provided without formal proof.

Lemma 24. Consider the economy specified in Example 1. The social weights satisfy $\bar{\alpha}_{2}\left(\delta_{2}^{\prime}, c_{0}^{\prime}\right)>\bar{\alpha}_{2}\left(\delta_{2}^{\prime}, c_{0}^{\prime}\right)$ if and only if $\varepsilon$ is below some threshold $\hat{\varepsilon} \approx 0.0167$.

In this example, the social weights are hence locally increasing if $G_{2}$ first-order stochastically dominates $G_{1}$ "sufficiently much". Loosely speaking, the workers in skill group 2 are on average worse off than the workers in skill group 1 in this case, because they have on average much higher fixed costs.

\section{B.4 Validity of Conditions 1 and 2 for specific functions}

In the following, I provide results on whether Conditions 1 and 2 are met if the groupspecific fixed costs distributions are given by some commonly used functional forms. Proofs and numerical simulations are available upon request.

Condition 1 (1i) requires the fixed cost distribution $G_{j}$ to be $\log$-concave for each $j \in J$.

Observation 1. For any $j \in J$, Condition 1 (1i) is satisfied if $G_{j}$ is given by

(a) a uniform distribution on $\left[\underline{\delta}_{j}, \bar{\delta}_{j}\right]$;

(b) a Pareto distribution with scale parameter (minimum value) $\underline{\delta}_{j}>0$ and shape parameter $k_{j}>0$;

(c) a log-normal distribution with location parameter $\xi_{j} \in \mathbb{R}$ and scale parameter $\sigma_{j}>0$; or

(d) a normal distribution with mean $\xi_{j} \in \mathbb{R}$ and standard deviation $\sigma_{j}>0$. 
Condition 11 (ii) refers to the co-variation of distributions $G_{j}$ and $G_{j+1}$ for each pair of skill groups $j$ and $j+1$. In particular, it assumes that the $c d f$ hazard rates can be unambiguously ordered for each $\delta \in \Delta, A_{j}(\delta) \geq A_{j+1}(\delta)$. In general, this assumption is neither stronger nor weaker then the assumption that $G_{j+1}$ first-order stochastically dominates $G_{j}$. Within each of the families of distribution functions considered here, however, both properties are equivalent.

Observation 2. For any $j \in J$, Condition 1 (ii) is satisfied and $G_{j+1}(\delta) \geq G_{j}(\delta)$ for all $\delta \in \Delta$ if the fixed cost distribution $G_{j}$ and $G_{j+1}$ are given by

(a) uniform distributions with upper endpoints $\bar{\delta}_{j} \geq \bar{\delta}_{j+1}$ and identical lower endpoints $\underline{\delta}_{j}=\underline{\delta}_{j+1} ;$

(b) Pareto distributions with shape parameters $0<k_{j} \leq k_{j+1}$ and identical scale parameters (minimum value) $\underline{\delta}_{j}=\underline{\delta}_{j+1}>0$;

(c) log-normal distributions with location parameters $\xi_{j} \geq \xi_{j+1} \in \mathbb{R}$ and identical scale parameters $\sigma_{j}=\sigma_{j+1}>0$;

(d) normal distributions with means $\xi_{j} \geq \xi_{j+1} \in \mathbb{R}$ and identical standard deviations $\sigma_{j}=\sigma_{j+1}>0$.

Finally, Condition 2 requires the $p d f$ hazard rate $a_{j}\left(\delta_{j}\right)$ to be weakly decreasing in $\delta$ and weakly increasing in $\omega$, but only at a sufficiently small rate compared to the derivative of the $c d f$ hazard rate $A_{j}\left(\delta_{j}\right)$.

Observation 3. For any $j \in J$, Condition 2 is satisfied

(a) for all $\delta \in \Delta$ if $G_{j}$ and $G_{j+1}$ are given by uniform distributions with upper endpoints $\bar{\delta}_{j} \geq \bar{\delta}_{j+1}$ and identical lower endpoints $\underline{\delta}_{j}=\underline{\delta}_{j+1}$;

(b) for all $\delta$ below some threshold level $z_{j}>\xi_{j}$ if $G_{j}$ and $G_{j+1}$ are given by normal distributions with means $\xi_{j} \geq \xi_{j+1}$ and identical standard deviations $\sigma_{j}=\sigma_{j+1}>0$.

Condition 2 is not satisfied if $G_{j}$ and $G_{j+1}$ are given by Pareto or log-normal distributions. In particular, Condition 2(i) is violated: the $p d f s$ of these distribution functions are not log-concave for any combination of parameters. I proceed by providing a relaxed version of the condition that is satisfied for these distribution functions. Recall that Condition 4 is used to prove the uniqueness of the fixed points $\underline{\beta}<1$ and $\bar{\beta}$ in Lemma 5 . The Lemma continues to hold if the joint type distribution satisfied the weaker Condition 4.

Condition 4. For every $j \in J /\{n\}, \delta \in \Delta$ and $x \in(0, \bar{\delta}-\delta)$, the joint type distribution satisfies

$$
B_{j}(\delta)\left[a_{j}(\delta)-a_{j+1}(\delta+x)\right]<\left(1+B_{j}(\delta)\right)\left[A_{j}(\delta)-A_{j+1}(\delta+x)\right],
$$

where $B_{j}(\delta):=A_{j}(\delta)\left[\delta^{*}\left(\omega_{j}\right)-\delta\right]$. 
Note that this condition is similar in spirit to Condition 22: Again, the $p d f$ hazard rate $a_{j}(\delta)$ is assumed to change at a sufficiently small rate compared to the $c d f$ hazard rate $A_{j}(\delta)$, both in $\delta$ and in $\omega$. For this relaxed condition, numerical simulations lead to the following observations.

Observation 4. For any $j \in J$, Condition 4 is satisfied

(a) for all $\delta$ above some threshold level $z_{j} \in\left(\underline{\delta}, \delta^{*}\left(\omega_{j}\right)\right)$ if $G_{j}$ and $G_{j+1}$ are given by Pareto distributions with shape parameters $0<k_{j} \leq k_{j+1}$ and identical scale parameters (minimum value) $\underline{\delta}_{j}=\underline{\delta}_{j+1}>0$;

(b) for all $\delta$ above some threshold level $z_{j} \in\left(0, \delta^{*}\left(\omega_{j}\right)\right)$ if $G_{j}$ and $G_{j+1}$ are given by lognormal distributions with location parameters $\xi_{j} \geq \xi_{j+1}$ and identical scale parameters $\sigma_{j}=\sigma_{j+1}>0$

(c) for all $\delta$ above some threshold level $z_{j}<\delta^{*}\left(\omega_{j}\right)$ if $G_{j}$ and $G_{j+1}$ are given by normal distributions with means $\xi_{j} \geq \xi_{j+1}$ and identical standard deviations $\sigma_{j}=\sigma_{j+1}>0$ and if $\delta \frac{g_{j}(\delta)}{G_{j}(\delta)}<1$.

For all functional forms considered here, the relaxed Condition 4 is satisfied for any $\delta \in$ $\left(\delta^{*}\left(\omega_{j}\right), \bar{\delta}\right)$, i.e., in the interval relevant for social weights that may induce the optimality of an EITC. Hence, Lemma 12 on the violation of the upwards IC constraint for sufficiently high weights $\alpha_{j}=\alpha_{j+1}>\bar{\beta}$ is ensured to hold.

\section{B.5 Upward distortions in multiple skill groups}

In Section 5, I define the set $\mathcal{A}^{U}$ of social weights for which optimal labor supply in skill group 2 is upwards distorted at both margins by Proposition 4 . Proposition 5 provides sufficient conditions for the existence of well-behaved welfare functions that give rise to welfare weights in the set $\mathcal{A}^{\mathcal{U}}$. In the following, I define subsets of $\mathcal{A}^{U}$ for which optimal labor supply in $l$ skill groups is ensured to be upwards distorted at both margins.

Definition 2. The set of weight sequences $\mathcal{A}_{l}^{U}$ contains all sequences $\alpha \in \mathcal{A}^{\chi}$ such that $\alpha_{j+1}>\beta_{j}^{U}\left(\alpha_{j}\right)$ for all $j \in\{1, \ldots, l-1\}$ and $\alpha_{j+1} \geq \beta_{j}^{D}\left(\alpha_{j}\right)$ for all $j \in\{l, \ldots, n-1\}$.

For each sequence of social weights in the set defined as this, we get the following result.

Proposition 6. For any $\alpha \in \mathcal{A}_{l}^{U}$, optimal output $y^{\alpha}$

- is upwards distorted at the intensive margin in each skill group $j \in\{2, \ldots, l\}$;

- is upwards distorted at the extensive margin in each skill group $j \in\{1, \ldots, l\}$.

Finally, I provide conditions that ensure the existence of well-behaved welfare functions for which $\bar{\alpha} \in \mathcal{A}_{l}^{U}$. 
Proposition 7. There are two numbers $a_{l}^{U}>1, m^{l}>l$ and two vectors $\left(\phi_{j}^{l}\right)_{j=1}^{n},\left(\tilde{\delta}_{j}^{l}\right)_{j=1}^{n}$ with $\phi_{j+1}^{l} \geq \phi_{j}^{l}$ for all $j \in J_{-n}, \phi_{j}^{l} \gtrless 1$ for $j \gtrless m^{l}$, and $\tilde{\delta}_{j}^{l} \in(\underline{\delta}, \bar{\delta})$ for all $j \in J$ such that, if

(a) $\frac{\omega_{j+1}}{\omega_{j}}<a_{l}^{U}$ for all $j \in\{1, \ldots, l-1\}$,

(b) $n \geq m^{l}$ and

(c) $\sum_{j=1}^{n} f_{j} G_{j}\left(\tilde{\delta}_{j}^{l}\right) \phi_{j}^{l}>1$,

there exists a regular combination of $\Psi$ and $\gamma$ for which $\bar{\alpha} \in \mathcal{A}_{l}^{U}$.

Note that the conditions are closely related to those in Proposition 5 . However, the threshold $m^{l}$ is weakly larger than threshold $m$ above. To ensure $\bar{\alpha} \in \mathcal{A}_{l}^{U}$ with $l>2$, there must hence be a sufficiently large share of workers with even higher skill types than for $\bar{\alpha} \in \mathcal{A}^{U}$. Besides, the relative distance between each pair of skill types below $\omega_{l}$ has to be sufficiently small. The proofs to Propositions 6 and 7 are available upon request; they apply the same arguments as the proofs of Propositions 4 and 5.

\section{B.6 Existence of social weights in other weight sets}

Proposition 5 above provides conditions under which the marginal welfare weights belong to set $\mathcal{A}^{U}$ for some regular combinations of $\Psi$ and $\gamma$. Put differently, it provides sufficient conditions for the existence of well-behaved welfare functions that are maximized by an EITC with negative marginal taxes and negative participation taxes. In the following, I clarify the conditions under some regular $(\Psi, \gamma)$ give rise to welfare weights in the sets $\mathcal{A}^{D}$ and $\mathcal{A}^{N}$.

By Proposition 8, there exist well-behaved welfare functions for which optimal labor supply is throughout undistorted at the intensive margin given any combination of the type set $\Omega \times \Delta$, the joint type distribution $K$ and the effort cost function $h$ that satisfy the previously imposed assumption. By Proposition 9, there exist well-behaved welfare functions for which a Negative Income Tax is optimal whenever the relative distance between all pairs of adjacent skill types is sufficiently small. In Appendix C, I study an example with commonly used functional forms, for which I also provide a necessary condition for the existence of regular $(\Psi, \gamma)$ such that $\bar{\alpha} \in \mathcal{A}^{D}$.

Proposition 8. There exist regular combinations of $\Psi$ and $\gamma$ for which $\bar{\alpha} \in \mathcal{A}^{N}$.

Proof. Assume that $\Psi$ is the identity function and fix some number $\hat{\delta} \in\left(\delta^{*}\left(\omega_{n},\right), \bar{\delta}\right)$. Consider the family of weighting functions $\gamma^{\varepsilon}$ with $\gamma^{\varepsilon}\left(\omega_{j}, \delta\right)=\gamma_{j}^{\varepsilon}=1-(j-1) \varepsilon$ for all $j \in J$ and $\delta<\hat{\delta}$, and $\gamma^{\varepsilon}\left(\omega_{j}, \delta\right)=\gamma_{0}=\left[1-\sum_{j=1}^{n} f_{j} G_{j}(\hat{\delta}) \gamma_{j}\right] /\left[\sum_{j=1}^{n} f_{j}\left(1-G_{j}(\hat{\delta})\right)\right]$ for all $j \in J$ and $\delta \geq \hat{\delta}$. For each $\varepsilon>0$, the average welfare weights are given by a decreasing sequence $\bar{\alpha}=\left(\bar{\alpha}_{0}, \bar{\alpha}_{1}, \ldots, \bar{\alpha}_{n}\right)$ with $\bar{\alpha}_{j}=\gamma_{j}^{\varepsilon}$ for all $j \in J$ and average weight 1 . For each 
$\varepsilon \in(0,1 /(n-1)), \bar{\alpha} \in \mathcal{A}^{\chi}$ because $\bar{\alpha}_{j} \in(0, \chi)$ for all $j \in J$. Moreover, $\bar{\alpha}_{2}<1$ ensures that $\bar{\alpha}_{j+1}<\beta_{j}^{U}\left(\bar{\alpha}_{j}\right)$ for all $j \in J_{-n}$. Consider the weights $\bar{\alpha}_{j}$ and $\bar{\alpha}_{j+1}$ for some $j \in J_{-n}$ and $\varepsilon \in(0,1 /(n-1))$. The difference between both weights is strictly increasing in $\varepsilon$, and the level of each weight is continuous and strictly decreasing in $\varepsilon$ for any $j>1$. For $\varepsilon=0$, $\bar{\alpha}_{j}=\bar{\alpha}_{j+1}=1$ and $\bar{\alpha}_{j+1}>\beta_{j}^{D}\left(\bar{\alpha}_{j}\right)$ by Lemma 13 . Additionally, Lemma 13 implies that there exists a unique number $\varepsilon_{j}>0$ for each $j \in J_{-n}$ such that $\bar{\alpha}_{j+1} \geq \beta_{j}^{D}\left(\bar{\alpha}_{j}\right)$ for all $\varepsilon \in\left(0, \varepsilon_{j}\right)$. Let $\hat{\varepsilon}:=\min _{j \in J_{-n}}\left\{\varepsilon_{j}\right\}$. For all $\varepsilon \in(0, \hat{\varepsilon}], \bar{\alpha} \in \mathcal{A}^{N}$.

Proposition 9. There is a number $a^{D}>1$ such that, if $\frac{\omega_{j+1}}{\omega_{j}}<a^{D}$ for all $j \in J_{-n}$, there exist regular combinations of $\Psi$ and $\gamma$ for which $\bar{\alpha} \in \mathcal{A}^{D}$.

Proof. Assume that $\Psi$ is the identity function and fix some number $\hat{\delta} \in\left(\delta^{*}\left(\omega_{n},\right), \bar{\delta}\right)$. Consider the family of weighting functions $\gamma^{\varepsilon}$ with $\gamma^{\varepsilon}\left(\omega_{j}, \delta\right)=\gamma_{j}^{\varepsilon}=(n-j+1) \varepsilon$ for all $j \in J$ and $\delta<\hat{\delta}$, and $\gamma^{\varepsilon}\left(\omega_{j}, \delta\right)=\gamma_{0}=\frac{1-\sum_{j=1}^{n} f_{j} G_{j}(\hat{\delta}) \gamma_{j}}{\sum_{j=1}^{n} f_{j}\left[1-G_{j}(\hat{\delta})\right]}$ for all $j \in J$ and $\delta \geq \hat{\delta}$. For each $\varepsilon \in(0,1 / n)$, the average welfare weights are given by a decreasing sequence $\bar{\alpha}=\left(\bar{\alpha}_{0}, \bar{\alpha}_{1}, \ldots, \bar{\alpha}_{n}\right)$ with $\bar{\alpha}_{j}=\gamma_{j}^{\varepsilon} \in(0,1)$ for all $j \in J$ and average weight 1 .

Let $a^{D}:=\min _{j \in J_{-n}}\left\{a_{j}^{0}\right\}$ and $\beta^{\prime}:=\min _{j \in J_{-n}}\left\{\underline{\beta}_{j}\right\}$, where $a_{j}^{0}$ and $\underline{\beta}_{j}$ are defined as in Lemmas 11 and 13 . If $\varepsilon \in\left(0, \beta^{\prime} / n\right)$, then $\bar{\alpha}_{j}<\underline{\beta}_{j}$ for all $j \in J_{-n}$. By Lemmas 11 and 13 , $\alpha_{j+1}<\beta_{j}^{D}\left(\alpha_{j}\right)$ for all $\alpha_{j+1}<\alpha_{j}<\underline{\beta}_{j}$ (for any $j \in J_{-n}$ ). Hence, the sequence of average welfare weights $\bar{\alpha}$ is an element of $\overline{\mathcal{A}}^{D}$ for any $\varepsilon \in\left(0, \beta^{\prime} / n\right)$.

\section{B.7 Illustration of optimally binding upward IC constraints}

In Subsection 6.3, I explain why optimal labor supply in skill group 2 is upwards distorted at the intensive margin for every social weight in the set $\mathcal{A}^{U}$. In particular, I argue that the upward IC constraint between skill groups 1 and 2 will always be binding in the optimal allocation. With respect to the IC constraints between skill groups 2 and 3, there are multiple possible constellations. In particular, the downward IC constraint between workers in both skill groups may be binding or slack in the optimal allocation. In both cases, however, labor supply in skill group 2 is upwards distorted at the intensive margin.

Figure 7 below illustrates both cases in panels 7(a) and 7(b). In both panels, the filled circles mark the bundles allocated to workers with skill types $\omega_{1}, \omega_{2}$ and $\omega_{3}$ in the solution to the relaxed problem. The indifference curves corresponding to these bundles are drawn as solid lines. As can be seen, workers with skill type $\omega_{1}$ prefer the bundle $\left(c_{2}^{\alpha R}, y_{2}^{\alpha R}\right)$ to the bundle $\left(c_{1}^{\alpha R}, y_{1}^{\alpha R}\right)$, while workers with skill type $\omega_{3}$ are indifferent between their own bundle and the bundle designed for workers with skill type $\omega_{2}$.

In the intermediate problem $A$, the IC constraints between workers with the two lowest skill types are taken into account (see Subsection 6.3 and proof to Proposition 4). The solution to this problem is represented by the empty circles and the corresponding dashed indifference curves for workers with skill types $\omega_{1}$ and $\omega_{2}$. As can be seen, the utility of workers in skill group 1 is higher than in the solution to the relaxed problem, while 


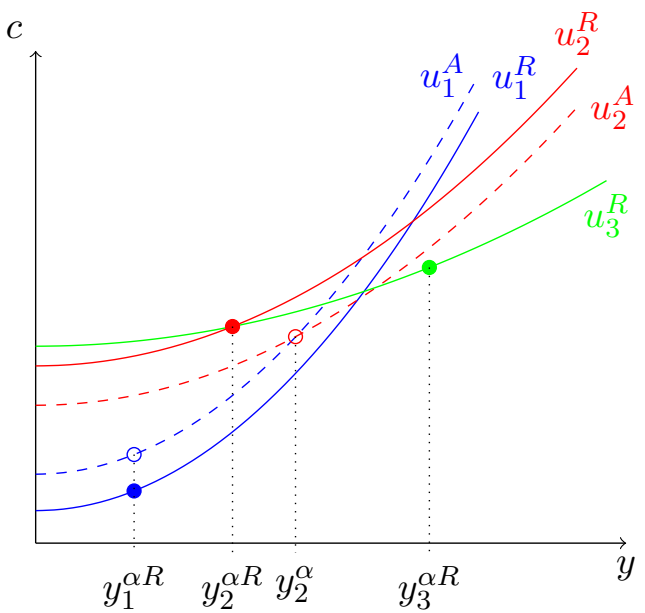

(a) The case with a slack downward IC constraint

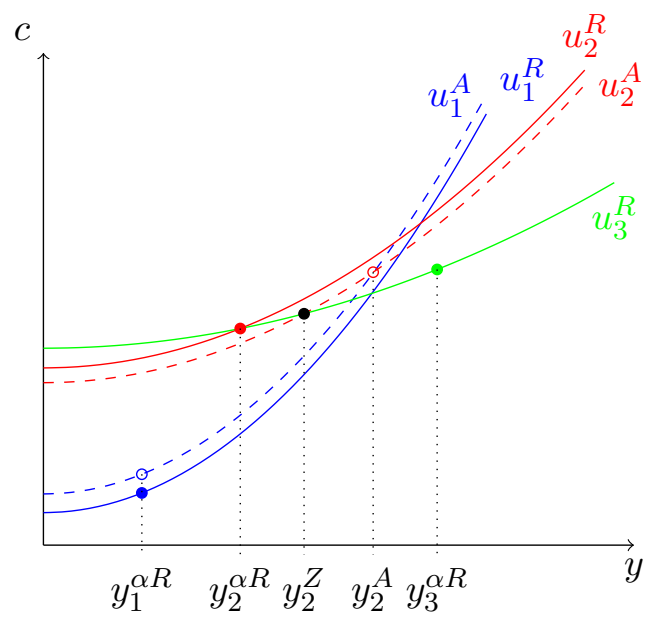

(b) The case with a binding downward IC constraint

Figure 7: Illustration of binding upward IC constraint for $\alpha \in \mathcal{A}^{U}$

the utility of workers in skill group 2 is lower. The output provided by workers in skill group 2 is strictly upwards distorted, $y_{2}^{A}>y_{2}^{\alpha R}$. In the case depicted in the left panel, the solution to the intermediate problem $A$ satisfies the downward IC constraint between workers in skill groups 2 and 3 . In this case, the solution to intermediate problem $A$ also solves the non-relaxed problem of optimal taxation. In the case depicted in the right panel, the solution to intermediate problem $A$ violates the downward IC constraint between the workers in groups 2 and 3. In the solution to the non-relaxed problem (not shown), this downward IC constraint will hence be binding. The optimal output level $y_{2}^{\alpha}$ will nevertheless be upwards distorted. More precisely, it will always be located between the output levels $y_{2}^{A}$ and $y_{2}^{Z}$ (corresponding to the intersection point $Z$ between the red dashed indifference curve of the workers in skill group 2 and the green indifference curve of the workers in skill group 3). Which of the two cases prevails, depends in general in a non-trivial way on the joint type distribution $K$, the effort cost function $h$ and the complete sequence of social weights $\alpha$.

\section{Example with commonly used functional forms}

The main results of this paper have been derived under relatively weak assumptions on the economy. In particular, I have not imposed any functional form assumptions (except for the quasi-linearity of utility in consumption). On the one hand, this demonstrates the generality of these results. On the other hand, the use of implicit functions makes it hard to assess how restrictive the Conditions in Proposition 5 are. The following section provides a closer look on a class of economies with simple and common specifications for the effort cost function $h$, the type set $\Omega \times \Delta$ and the type distribution $K$. For these functional forms, simple closed-form expressions can be derived. 
Condition 5. The economy satisfies the following assumptions:

(i) The effort cost function is given by $h(y, \omega)=\frac{1}{1+1 / \sigma}\left(\frac{y}{\omega}\right)^{1+1 / \sigma}$ with $\sigma>0$,

(ii) the skill space is given by the finite set $\left\{\omega_{1}, \omega_{2}, \ldots, \omega_{n}\right\}$ with constant relative distances $\frac{\omega_{j+1}}{\omega_{j}}=a>1$ for each $j \in J /\{n\}$,

(iii) the fixed cost space is given by the interval $[0, \bar{\delta}]$ with $\bar{\delta}>\frac{1}{\sigma+1} \omega_{n}^{\sigma+1}$, and

(iv) for each $j \in J$, the conditional distribution $G_{j}$ of fixed costs is given by a uniform distribution on $[0, \bar{\delta}]$.

By the first part of Condition 5, the utility function has a commonly used functional form that ensures a constant elasticity of hours worked, i.e., a constant elasticity of labor supply $y_{T}(\omega, \delta) / \omega$ with respect to the net-of-tax rate $\left[1-T^{\prime}(y)\right] \omega$, conditional on participation in the labor market. In particular, this elasticity equals the parameter $\sigma$ for all working types. By the second part, the relative distance between each pair of adjacent skill types is identical and equal to parameter $a$. The third part specifies the lower endpoint $\underline{\delta}=0$ of the fixed cost set $\Delta$, and establishes a lower bound on the upper endpoint $\bar{\delta}$. By part (iv), finally, fixed costs are uniformly distributed on $\Delta$ in each skill group. This implies that the semi-elasticity $\eta_{j}$ of participation is equal to $1 / \delta_{j}$ for each $j \in J$. Note also that skill types and fixed cost types are independently distributed. Under Condition 5 , an economy is uniquely defined by the parameters $\sigma, a, n, \bar{\delta}$, and by the skill distribution $F$.

The proofs to the following Lemmas are available upon request.

Lemma 25. Under Condition 5, Conditions 1 and 3 are satisfied for all combinations of $\sigma, a, n$ and $\bar{\delta}$. The optimal tax problem has a unique interior extremum for all weight sequences $\alpha$ such that $\alpha_{j}<\chi=2$ for all $j \in J$.

Lemma 26. Under Condition 5 ,

(i) $\mathcal{A}^{D}$ is non-empty if a is below the threshold e $(\sigma)$ defined by

$$
e(\sigma)=x>1: x^{\sigma+1}-1-2 \sigma\left(1-x^{-(1+1 / \sigma)}\right)=0,
$$

(ii) there is a vector $\left(\phi_{j}\right)_{j=1}^{n}>0$ and a number $\kappa>0$ such that $\mathcal{A}^{U}$ is non-empty if and only if

$$
\begin{aligned}
n & >\bar{m}(a, \sigma):=2+\frac{\ln \left(\frac{\sigma\left(\tilde{a}^{1 / \sigma}-1\right)\left(\tilde{a}^{-1 / \sigma}-\tilde{a}\right)}{\sigma\left(1-\tilde{a}^{-1 / \sigma}\right)+1-\tilde{a}}\right)}{\ln \tilde{a}}, \\
\bar{\delta} & <d(a, \sigma, n):=\psi(a, \sigma, n) \frac{\omega_{n}^{\sigma+1}}{\sigma+1} \text { and } \\
\sum_{j=1}^{n} f_{j} \phi_{j} & >\kappa,
\end{aligned}
$$




$$
\text { where } \tilde{a}=a^{\sigma+1} \text { and } \psi(a, \sigma, n):=\min \left\{\frac{1-\frac{\tilde{a}+\tilde{a}^{-1 / \sigma}\left(\tilde{a}^{n-2}-1\right)}{\tilde{a}^{n-1}}}{1-\frac{\tilde{\tilde{c}}-1}{\sigma \tilde{a}\left(\tilde{a}^{1 / \sigma}-1\right)}}, \frac{2 \sigma \tilde{a}\left(\tilde{a}^{1 / \sigma}-1\right)-\tilde{a}+1}{2\left[\sigma \tilde{a}\left(\tilde{a}^{1 / \sigma}-1\right)-\tilde{a}+1\right]}\right\}
$$

Lemma 26 refines Propositions 5 and 9 for the family of economies defined by Condition 5. It provides necessary and sufficient conditions for the existence of social weights in the sets $\mathcal{A}^{D}$ and $\mathcal{A}^{U}$, expressed in terms of the parameters mentioned above. Notably, these conditions are simple threshold conditions on the parameters $a, n$, and $\bar{\beta}$ and the skill distribution $F$. Table 1 reports the levels of these thresholds for specific values for the intensive-margin elasticity $\sigma$ and the relative skill distance $a$. In the following, I comment on the threshold levels for my preferred scenario with $\sigma=.3$ and $a=1.1$. To show the qualitative robustness of my results, Table 1 additionally provides the levels for $\sigma=.5$ and $a=1.5$.

The first three lines of Table 1 illustrate part (ii) of Lemma 26, which provides sufficient conditions for the non-emptiness of set $\mathcal{A}^{U}$. Recall that this condition is satisfied if and only if there is a sufficiently large mass of working types with skills above a threshold $\omega_{m}$. For the class of economies considered here, this is equivalent to assuming that, first, the cardinality $n$ of the skill set $\Omega$ is above some the threshold $m(a, \sigma)$, second, that the maximum level $\bar{\beta}$ of fixed costs is below the threshold $\bar{\beta}(a, \sigma, n)$, and third, that the population share of agents with skill types above $\omega_{m}$ is sufficiently large. For my preferred scenario, the first condition is satisfied if there are at least 9 skill groups $(m=8.97)$. This implies that agents with the highest skill type $\omega_{n}$ must be about two and a half times as productive as the lowest skill group $\left(\omega_{m} / \omega_{1}=2.35\right)$. The second condition ensures that a majority of high-skill agents participates in the labor market, given the assumed uniform distribution of fixed costs. Table 1 does not report the level of the threshold $d(a, \sigma, n)$. Instead, I report a transformed version of this threshold that is more readily interpretable. In particular, I provide the minimum level $\gamma_{20}(a, \sigma)$ of the participation share in the highest skill group for $n=20$. For my preferred scenario, more than 54 percent of the agents with the highest skill type have to participate in the labor market in the optimal allocation 52

The fourth line of Table 1 illustrates part (i) of Lemma 26, which provides a necessary condition for the non-emptiness of set $\mathcal{A}^{D}$. Recall that this condition is satisfied if and only if the relative distance between each pair of adjacent skill types is small enough. For the preferred level of the elasticity $\sigma=0.3$, this is true whenever an agent with any skill types $\omega_{j}$ is less than 30 percent more productive than an agent with the next-lower skill type $\omega_{j-1}$ (parameter $a$ is below $e(.3)=1.3$ ). For the class of economies defined by Condition 5, it is also possible to provide a necessary condition for the non-emptiness of $\mathcal{A}^{D}$.

The remaining lines of Table 1 provide numerical values for the thresholds $\underline{\beta}$ and $\bar{\beta}$ in

\footnotetext{
${ }^{52}$ To see the equivalence between the thresholds $d$ and $\gamma$, note that $G_{n}\left(\hat{\delta}_{n}\right)=\frac{q_{n}\left(\beta_{n}\right)}{\delta}$ and $\gamma_{n}(a, \sigma)=\frac{q_{n}\left(\beta_{n}\right)}{d(a, \sigma, n)}$.
} 


\begin{tabular}{|l||c|c|c||c|c|c|}
\hline \multicolumn{1}{|c||}{} & \multicolumn{3}{c||}{$\sigma=.3$} & \multicolumn{3}{c|}{$\sigma=.5$} \\
& $a=1.5$ & $a=1.1$ & $a \rightarrow 1$ & $a=1.5$ & $a=1.1$ & $a \rightarrow 1$ \\
\hline$m(a, \sigma)$ & 5.02 & 8.97 & $\infty$ & 4.18 & 7.73 & $\infty$ \\
$\omega_{m} / \omega_{1}$ & 7.65 & 2.35 & 2 & 5.45 & 2.09 & 2 \\
$\gamma_{20}(a, \sigma)$ & .42 & .54 & - & .38 & .51 & - \\
\hline$d(\sigma)$ & 1.30 & 1.30 & 1.30 & 1.37 & 1.37 & 1.37 \\
\hline $\bar{\beta}$ & 1.72 & 1.24 & 1 & 1.61 & 1.20 & 1 \\
$\underline{\beta}$ & $<0$ & .70 & 1 & $<0$ & .76 & 1 \\
\hline$\kappa$ & .72 & .24 & 0 & .61 & .20 & 0 \\
$\phi_{1}^{\prime}=\phi_{2}$ & 0 & 0 & 0 & 0 & 0 & 0 \\
$\phi_{3}^{\prime}$ & .17 & .04 & 0 & .24 & .04 & 0 \\
$\phi_{4}^{\prime}$ & .41 & .08 & 0 & .56 & .08 & 0 \\
$\phi_{20}^{\prime}$ & 1.72 & .45 & 0 & 1.61 & .39 & 0 \\
$\phi_{\infty}^{\prime}$ & 1.72 & .54 & - & 1.61 & .44 & - \\
\hline
\end{tabular}

Table 1: Numerical values for different combinations of $\sigma$ and $a$

Lemma 5 and for the vector $\phi=\left(\phi_{j}\right)_{j=1}^{n}=\kappa^{-1}\left(\phi_{j}^{\prime}\right)_{j=1}^{n}$ in Lemma 26 (and in Proposition 5.)

Lemma 27. Let Assumption 5 be satisfied and $n=2$. There is a number $a^{N}(\sigma) \in$ $\left(1,(2+2 \sigma)^{1 /(1+s)}\right)$ such that optimal output $y_{\alpha}$ in both skill groups is undistorted at the intensive margin for each $\alpha \in \mathcal{A}^{\chi}$ if

$$
\begin{aligned}
a & \geq a^{N}(\sigma), \text { and } \\
f_{1} & \geq \frac{1}{2}\left(1-\frac{1}{\tilde{a}-2 \sigma\left(1-\tilde{a}^{-1 / \sigma}\right)}\right)^{-1} \in(0,1) .
\end{aligned}
$$

For an elasticity of $\sigma=.3$, the threshold $a_{u}(\sigma)$ is given by 2.07 . Hence, optimal labor supply is undistorted at the intensive margin for any decreasing weight sequence in an economy where (a) there are only two skill levels, (b) high-skilled agents are more than 2.07 times as productive as low-skilled agents, and (c) the share of low-skilled agents is large enough.

Finally, Condition 5 allows to study how the main results depend on the assumption of a finite skill space. In particular, assume that the relative skill distances a converges from above to 1 , i.e., that the finite skill space converges to an interval. Table 1 provides the thresholds for this limiting case as well. For this limit, two conclusions can be drawn. First, set $\mathcal{A}^{N}$ vanishes in the limit. Hence, the optimal allocation involves distortions at the intensive margin for any decreasing weight sequence. In particular, as the threshold $\underline{\beta}$ converges to 1 , labor supply is necessarily downwards distorted at the intensive margin for 
all working types with weights below the population average. Second, this does not imply that the potential optimality of upward distortions at both margins vanishes. Instead, the conditions in part (ii) of Lemma 26 (and Proposition 5) become easier to satisfy. In particular, the relative distance $\frac{\omega_{m}}{\omega_{1}}$ has to exceed the same threshold for all levels of $\sigma>0$.

Lemma 28. Let Assumption 5 be satisfied and let the relative distance a converge to 1 from above. Then, the relative distance between skill types $\omega_{m(a, \sigma)}$ and $\omega_{1}$ converges to $\lim _{a \rightarrow 1} \omega_{m(a, \sigma)} / \omega_{1}=2$ for all $\sigma>0$. 